\title{
Some exactly solvable models of urn process theory
}

\author{
Philippe Flajolet, Philippe Dumas, and Vincent Puyhaubert
}

Algorithms Project, INRIA, F-78153 Le Chesnay (France)

We establish a fundamental isomorphism between discrete-time balanced urn processes and certain ordinary differential systems, which are nonlinear, autonomous, and of a simple monomial form. As a consequence, all balanced urn processes with balls of two colours are proved to be analytically solvable in finite terms. The corresponding generating functions are expressed in terms of certain Abelian integrals over curves of the Fermat type (which are also hypergeometric functions), together with their inverses. A consequence is the unification of the analyses of many classical models, including those related to the coupon collector's problem, particle transfer (the Ehrenfest model), Friedman's "adverse campaign" and Pólya's contagion model, as well as the OK Corral model (a basic case of Lanchester's theory of conflicts). In each case, it is possible to quantify very precisely the probable composition of the urn at any discrete instant. We study here in detail "semi-sacrificial" urns, for which the following are obtained: a Gaussian limiting distribution with speed of convergence estimates as well as a characterization of the large and extreme large deviation regimes. We also work out explicitly the case of 2-dimensional triangular models, where local limit laws of the stable type are obtained. A few models of dimension three or greater, e.g., "autistic" (generalized Pólya), cyclic chambers (generalized Ehrenfest), generalized coupon-collector, and triangular urns, are also shown to be exactly solvable.

\section{Contents}

$\begin{array}{lll}1 & \text { Urns and differential systems } & 61\end{array}$

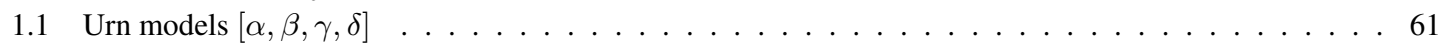

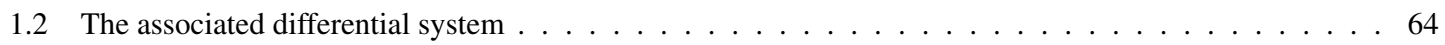

$2 \quad$ Basic examples of $2 \times 2$ urns with entries in $\{0, \pm 1\}$

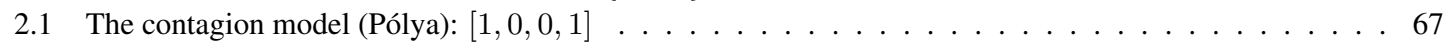

2.2 The adverse-campaign model (Friedman): $[0,1,1,0] \ldots \ldots \ldots \ldots \ldots$

2.3 The two-chambers urn (Ehrenfest): $[-1,1,1,-1] \ldots \ldots \ldots \ldots \ldots$

2.4 The coupon collector's urn: $[-1,1,0,0] \ldots \ldots \ldots \ldots \ldots \ldots \ldots \ldots \ldots \ldots$

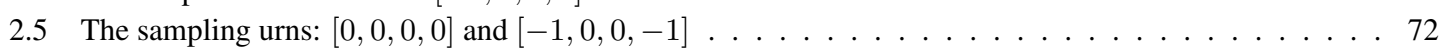

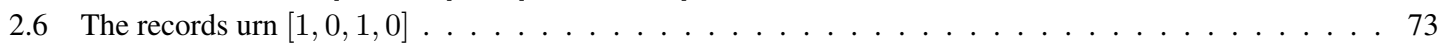

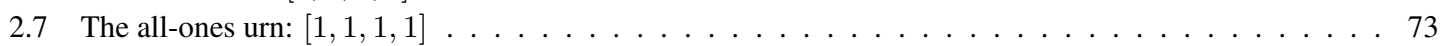

\begin{tabular}{|lll}
\hline 3 & The first integral of balanced $2 \times 2$ urns & 74
\end{tabular}

\begin{tabular}{|l|}
\hline General solutions for $2 \times 2$ sacrificial urns $(\alpha \leq-1)$ \\
\hline
\end{tabular}

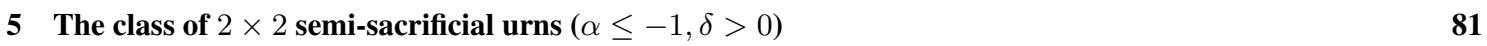

5.1 The analytic structure of the base functions $S, C \ldots \ldots \ldots \ldots \ldots \ldots$

5.2 Probabilistic properties of semi-sacrificial urns $\ldots \ldots \ldots \ldots \ldots \ldots \ldots$

$5.2 .1 \quad$ Extreme large deviations $\ldots \ldots \ldots \ldots \ldots \ldots \ldots \ldots$

5.2 .2 A Gaussian limit law with speed $\ldots \ldots \ldots \ldots \ldots \ldots$

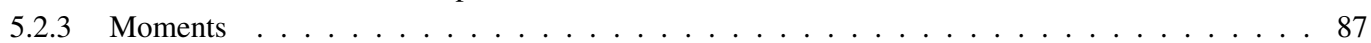

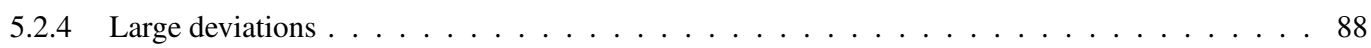

5.3 The algebraic family $[-1, \sigma+1, \sigma-1,1] \ldots \ldots \ldots \ldots \ldots \ldots \ldots \ldots \ldots$

5.4 The algebraic family $[-1, \sigma+1,1, \sigma-1](\sigma$ even $) \ldots \ldots \ldots \ldots \ldots \ldots$

$6 \quad$ The class of $2 \times 2$ fully sacrificial urns $(\alpha, \delta<-1)$

6.1 Analytic solutions $\ldots \ldots \ldots \ldots \ldots \ldots \ldots$

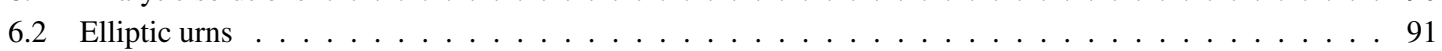

1365-8050 (C) 2006 Discrete Mathematics and Theoretical Computer Science (DMTCS), Nancy, France 


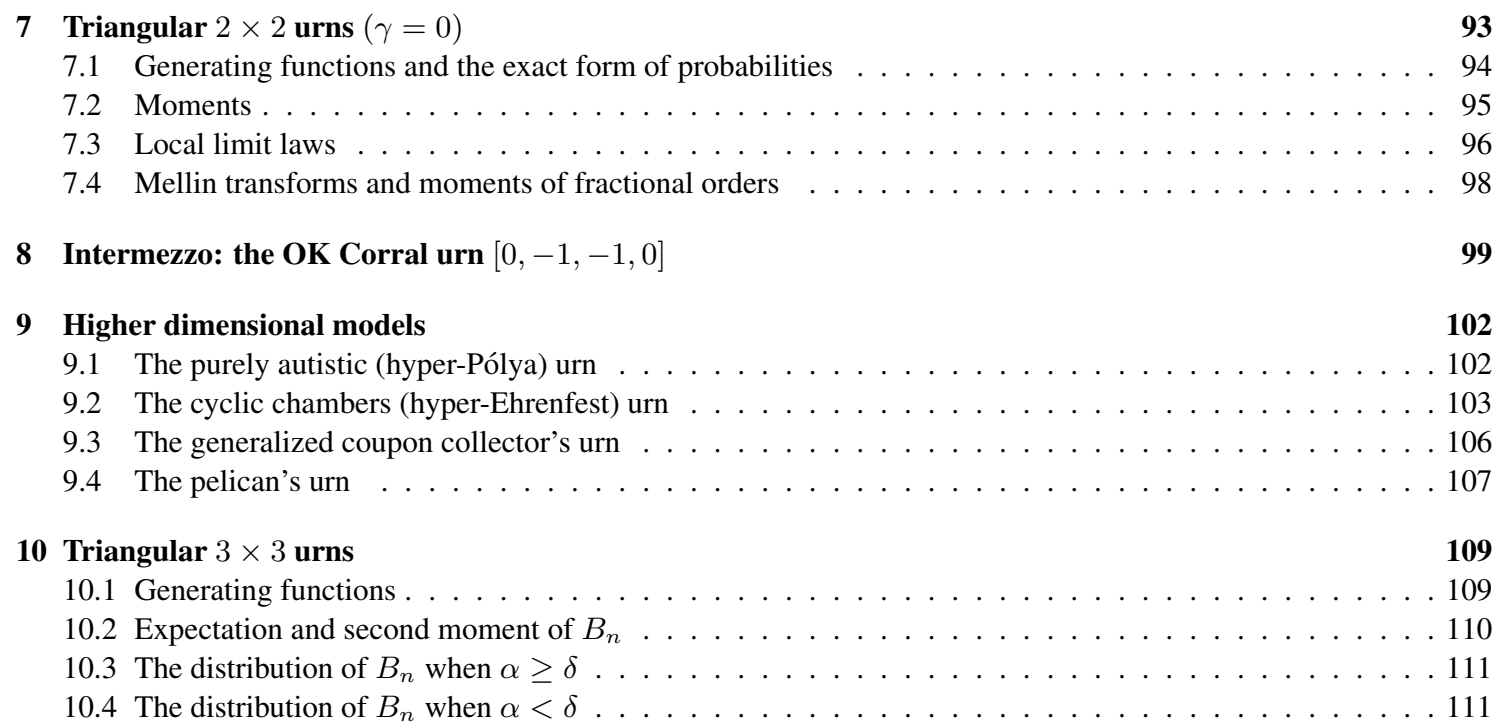

11 Relations between discrete and continuous time models

\section{Introduction}

The urn models which we consider are the ones where a single urn contains balls of different colours and a fixed set of rules specifies the way the urn composition evolves: at each discrete instant, a ball is picked up at random, its colour is inspected, and, in compliance with the rules, a collection of balls of various colours is added to the urn. In this study, we restrict attention to balanced urn models, where the total number of balls added at any instant is a deterministic quantity. Such urn models have been intensely studied for about three centuries: early contributions are due to Jacob Bernoulli and Laplace [75], the subject being then largely renewed by works of Pólya in the first half of the twentieth century. The book by Johnson and Kotz [64] (see also [73] for a more recent update) offers an interesting perspective on the simplest models, their history, and some of their applications in statistics, engineering, and genetics. For dimension 2 (i.e., balls can be of either of two colours), the model is precisely described in 1.1 below.

Our main theme is the following. To any balanced urn model, one can associate directly an ordinary differential system of the same dimension. This differential system has particular features, in that it is autonomous, monomial, and homogeneous in degree. The algebra of such a simple differential system turns out to be isomorphic to the combinatorics of a balanced urn model. In particular an explicit solution of the differential systems provides automatically an analytic solution of the urn model. We develop this fundamental property in $\$ 1.2$ in the case of dimension 2 and later extend it in $\$ 9$ to the case of urns with more than two colours.

The fundamental isomorphism between balanced urn models and differential systems makes it possible to derive in a direct and unified way most of the explicit solutions known for special urns with two coloursfor instance those of Pólya, Ehrenfest, Friedman, as well as the coupon collector's urn: this is the subject of $\$ 2$ Perhaps a more important consequence is that balanced urns with two colours belong to the class of integrable systems: we establish in 3 that the differential systems, for dimension 2 at least, always admit of an explicit first integral, so that the associated generating functions can be constructed from a finite sequence of elementary algebraic operations combined with quadratures and functional inversion (this corresponds to "closed-form" functions in the terminology of Taylor [98]). These integrability results extend those obtained earlier by Flajolet, Gabarró, and Pekari [30], where the method of characteristics was used to solve an associated partial differential equation. The new feature of treating urn models directly via an ordinary differential system takes some of its roots in the recent combinatorial study [16].

As an illustration of the usefulness of explicit solutions, we examine in detail in $\$ 4$ and $\$ 5$ the class of semi-sacrificial urns of dimension 2, which are defined by the fact that one diagonal entry is positive, the other negative. For such urns, we obtain the following: a Gaussian limit law for the urn's composition at large instants; a characterization of the speed of convergence to the Gaussian limit; a quantification of the extreme large deviation regime; the large deviation rate function; the structure of moments. These properties extend what was known earlier from [30] regarding fully sacrificial urns, where both diagonal 
entries are negative. The results of [30] are briefly reviewed in $\$ 6$, which also contains a brief discussion of $2 \times 2$ urns whose solution involves elliptic functions.

Next we treat triangular urns of dimension 2 in $\$ 7$, where we report on unpublished results that parallel and supplement a recent independent study of Janson [63]. Section 8 is an intermezzo dedicated to the OK Corral problem: there, a natural time-reversal transformation applied to Friedman's urn (the adversecampaign model) combines in an interesting fashion with analytic methods and provides exact evaluations as well as asymptotic estimates that appear to be new.

In dimension greater than two, the isomorphism with differential systems still holds; see $\$ 9$ However, the systems are not in general (explicitly) integrable. We can nonetheless single out a few special classes of exactly solvable urn models with dimension three or more, including generalized Pólya and generalized coupon collector's urns, as well as a multi-chamber Ehrenfest model: see \$9, triangular urns of dimension 3 are also (somewhat) exactly solvable: we report preliminary results on these in $\$ 10$

Our methodology strongly relies on special functions, singularity analysis, quasi-powers approximations, and, more generally methods exposed in the forthcoming book Analytic Combinatorics [38]. The principle of looking for explicit solutions could hardly be better expressed than by the following quotation from Eilbeck, Mikhailov, Santini, and Zakharov [in an introduction to a semester on Integrable Systems, at the Newton Institute in Cambridge, 2001]:

Rather surprisingly, relatively sizable classes of nonlinear systems are found to have an extra property, integrability, which changes the picture completely. Integrable systems have a rich mathematical structure, which means that many interesting exact solutions to the PDEs can be found. Although important in their own right, these systems form an archipelago of solvable models in a sea of unknown, and can be used as stepping stones to investigate properties of "nearby" non-integrable systems.

(Our title is inspired by that of Baxter's magnificent opus, Exactly solved models in statistical physics [8].) As we propose to demonstrate in subsequent works, the methods of analytic combinatorics can be further adapted to several urn models that no longer admit of explicit solutions.

Apart from works that treat isolated problems, we mention here a few researches that deal with urn models at a level comparable to the one we are aiming at here. Friedman [43] launched the study of models determined by a general symmetric $2 \times 2$ (balanced) matrix. Subsequently, Bagchi and Pal [6] elucidated the structure of moments for balanced $2 \times 2$ urns and deduced a Gaussian limit law for a large class of models. Smythe [96] found a theorem of considerable generality based on the spectral properties of the urn's matrix, which is applicable to arbitrary dimensions. Gouet [46, 47, 48] developed powerful martingale methods that lead to functional limit theorems, but are often of a nonconstructive (i.e., nonexplicit) nature. Pouyanne [91, 90] has recently developed a widely applicable operator approach to the determination of moments and it would be of great interest to relate his work to the present study. Higueras et al. [52] elaborate on the asymptotic theory of urns via algebraic differential equations, in a way that leads to results of appreciable generality whenever some almost sure convergence property is a priori known to hold. Finally, a special mention is due to the works of Athreya, Karlin, and Ney [3, 4] based on an embedding into continuous time, which has the capacity of dealing with nonbalanced urn models (see also [2]); a central contribution in this area is Janson's recent study [62].

Our interest in this range of questions started from problems in discrete probability theory and the analysis of algorithms. For these aspects, we refer to Mahmoud's survey [84].

This text is an expanded version of invited lectures given by P. Flajolet at the Workshop on Analysis of Algorithms (AOFA2006, Alden Biesen, Belgium, 2-8 July 2006) and at the Fourth Colloquium on Mathematics and Computer Science-Algorithms, Trees, Combinatorics and Probabilities (Nancy, France, September 18-22, 2006). It represents to a large extent a report on work in progress mingled with an attempted survey of the field of urn processes examined under the perspective of exactly solvable models and analytic combinatorics methods.

\section{Urns and differential systems}

\subsection{Urn models $[\alpha, \beta, \gamma, \delta]$}

We consider here urns that may contain balls of two possible colours or types, which we call rather unpoetically $x$ and $y$. An urn model is specified by a matrix with integer entries,

$$
\mathcal{M}=\left(\begin{array}{cc}
\alpha & \beta \\
\gamma & \delta
\end{array}\right), \quad \alpha, \delta \in \mathbb{Z}, \beta, \gamma \in \mathbb{Z}_{\geq 0},
$$


also abbreviated as $[\alpha, \beta, \gamma, \delta]$. At time 0 , the urn contains initially

$$
a_{0} \text { balls of type } \mathrm{x}, \quad b_{0} \text { balls of type } \mathrm{y} .
$$

At regularly spaced discrete instants, the urn evolves according to the following rule:

Urn evolution rule. A ball is chosen from the urn, with all balls present in the urn at that time having equal chances of being chosen, and its colour is inspected (i) If the ball chosen is of colour $x$, then $\alpha$ new balls of type $\mathrm{x}$ and $\beta$ new balls of type $\mathrm{y}$ are placed into the urn; if the ball chosen is of colour $\mathrm{y}$, then $\gamma$ new balls of type $\mathrm{x}$ and $\delta$ new balls of type $\mathrm{y}$ are placed into the urn.

By convention, a negative (diagonal) entry in the matrix $\mathcal{M}$ means that balls of the corresponding colour should be taken out of the urn.

For instance, with the matrix $\mathcal{M}=\left(\begin{array}{cc}-1 & 3 \\ 2 & 0\end{array}\right)$, a possible evolution starting with the initial configuration $\left(a_{0}, b_{0}\right)=(3,1)$, at time 0 , is described by the following sequence

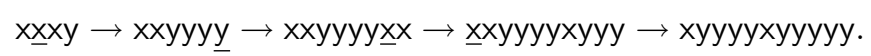

There, the ball chosen at each step is underlined. If it is $x$, then this ball is removed from the urn (since $\alpha=-1$ ) and 3 balls of type $\mathrm{y}$ are appended (since $\beta=3$ ); if it is $\mathrm{y}$, then then 2 balls of type $\mathrm{x}$ are appended and the balls of type y are kept untouched (since $\gamma=2$ and $\delta=0$ ). We call history any such unambiguous description of the evolution of an urn-a history is thus nothing but a complete description of a sample path (or trajectory) of the urn process. The length of a history is the number of transitions ( $\rightarrow$ arrows) that it comprises, so that a history of length $n$ specifies the possible evolution (ii) of an urn from time 0 to time $n$. This paper is entirely based on developing an enumerative approach to urn histories, in contrast to probabilistic treatments on which most publications in the field rely.

Throughout this article, we limit ourselves to considering urns that are balanced in the sense that the matrix $\mathcal{M}$ has constant row sums, this sum being consistently denoted by $\sigma$,

$$
\sigma=\alpha+\beta=\gamma+\delta
$$

and called the balance. For an urn which is balanced, the number $s_{n}$ of balls that it contains at discrete time $n$ is a deterministic quantity satisfying

$$
s_{n}=a_{0}+b_{0}+n \sigma=s_{0}+n \sigma .
$$

For urns with negative diagonal entries, there is also an issue of tenability. Say we have $\alpha=-g$ with $g \geq 2$. This means that, whenever an $\mathrm{x}$-ball is picked up, a group of $g$ balls should be taken out of the urn. In order to avoid configurations that would block the urn, one must postulate that the initial composition of the urn satisfies $g \mid a_{0}$ and that the number of balls of the first kind added when a ball of the second kind is chosen satisfies $g \mid \gamma$. Similarly for y-balls.

Definition 1 An urn given by a matrix $\mathcal{M}$ is tenable when the following two conditions are met:

(i) if the diagonal entry $\alpha$ is such that $\alpha<-1$, then $|\alpha|$ divides both $a_{0}$ and $\gamma$;

(ii) if the diagonal entry $\delta$ is such that $\delta<-1$, then $|\delta|$ divides both $b_{0}$ and $\beta$.

Henceforth, all urn models involving negative diagonal entries are assumed to be tenable.

Our global purpose is to determine the composition of the urn at any given time $n$. Let $\mathcal{H}_{n}\left(\begin{array}{l}a_{0} \\ b_{0}\end{array}\right)$ be the set of histories of length $n$ when the urn has the initial configuration $\left(a_{0}, b_{0}\right)$, and $\mathcal{H}_{n}\left(\begin{array}{l}a_{0} a \\ b_{0} b\end{array}\right)$ the subset of those which, at time $n$, correspond to an urn with $a$ balls of type $\mathrm{x}$ and $b$ balls of type $\mathrm{y}$. The corresponding cardinalities are

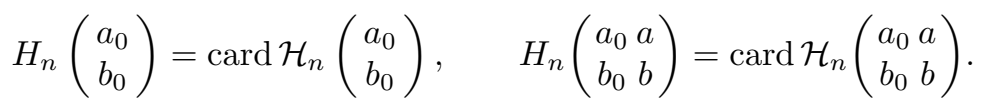

\footnotetext{
(i) Thus, the chosen ball is not removed from the urn: this is the convention adopted by virtually all authors in the field, one that entails no loss of generality.

(ii) We may regard the transitions as occurring at half integer times, or, equivalently, one may consider the $n$th transition to occur immediately before time $n$.
} 
We shall approach the determination of these quantities via their generating functions (GFs):

$$
H\left(z \mid \begin{array}{l}
a_{0} \\
b_{0}
\end{array}\right)=\sum_{n} H_{n}\left(\begin{array}{l}
a_{0} \\
b_{0}
\end{array}\right) \frac{z^{n}}{n !}, \quad H\left(x, y, z \mid \begin{array}{l}
a_{0} \\
b_{0}
\end{array}\right)=\sum_{n, a, b} H_{n}\left(\begin{array}{l}
a_{0} a \\
b_{0} b
\end{array}\right) x^{a} y^{b} \frac{z^{n}}{n !},
$$

here taken to be of the exponential type. The trivariate GF is also called the complete generating function of urn histories. For future reference, we state:

Proposition 1 For an urn of balance $\sigma$ with $\sigma>0$, the number of histories of length $n$ satisfies

$H_{n}\left(\begin{array}{l}a_{0} \\ b_{0}\end{array}\right)=s_{0}\left(s_{0}+\sigma\right) \cdots\left(s_{0}+(n-1) \sigma\right)=\sigma^{n} \frac{\Gamma\left(n+s_{0} / \sigma\right)}{\Gamma\left(s_{0} / \sigma\right)}=n ! \sigma^{n}\left(\begin{array}{c}n+s_{0} / \sigma-1 \\ n\end{array}\right), \quad s_{0}:=a_{0}+b_{0}$,

and the corresponding exponential generating function is

$$
H\left(z \mid \begin{array}{l}
a_{0} \\
b_{0}
\end{array}\right)=\frac{1}{(1-\sigma z)^{\left(a_{0}+b_{0}\right) / \sigma}} \quad(\sigma>0) .
$$

The complete generating function of urn histories $H\left(x, y, z \mid \begin{array}{l}a_{0} \\ b_{0}\end{array}\right)$ is, for any $R \geq 1$, analytic in the domain

$$
|x| \leq R, \quad|y| \leq R, \quad|z|<\frac{1}{\sigma R^{\sigma}}
$$

An immediate consequence of Stirling's formula is

$$
H_{n} \equiv H_{n}\left(\begin{array}{l}
a_{0} \\
b_{0}
\end{array}\right)=n ! \sigma^{n} \frac{n^{s_{0} / \sigma-1}}{\Gamma\left(s_{0} / \sigma\right)}\left(1+\frac{s_{0}\left(s_{0}-\sigma\right)}{2 \sigma^{2} n}+O\left(\frac{1}{n^{2}}\right)\right), \quad s_{0}=a_{0}+b_{0} .
$$

Proof: The number of histories is determined by the observation that there are $s_{0}$ possible ways to pick up a ball at step $1, s_{1}=s_{0}+\sigma$ ways at time 2, and so on. The univariate GF then plainly results from the binomial theorem. For $|x| \leq R$ and $|y| \leq R$, the trivariate GF $H(x, y, z)$ has the coefficient of its $z^{n}$ term dominated by

$$
\left(s_{0}+\sigma n\right) R^{s_{0}+\sigma n} \cdot \sigma^{n} \frac{\Gamma\left(s_{0} / \sigma+n\right)}{\Gamma\left(s_{0} / \sigma\right) \Gamma(n+1)}=O\left(R^{\sigma n} \sigma^{n} n^{s_{0} / \sigma}\right)
$$

from which the analyticity property results.

In what follows, we shall mostly use the short forms

$$
H_{n} \equiv H_{n}\left(\begin{array}{c}
a_{0} \\
b_{0}
\end{array}\right), \quad H(z) \equiv H\left(z \mid \begin{array}{c}
a_{0} \\
b_{0}
\end{array}\right), \quad H(x, y, z) \equiv H\left(x, y, z \mid \begin{array}{c}
a_{0} \\
b_{0}
\end{array}\right),
$$

whenever the initial conditions (the values of $a_{0}, b_{0}$ ) are understood from context.

The univariate GF $H(z)$ of all histories is by (5) a simple algebraic function. The trivariate (complete) GF $H(x, y, z)$ is the one that determines the urn composition; it is a (yet unknown) deformation of $H(z)$, and Proposition 1 ensures that it is always a function of $z$ analytic in a neighbourhood of the origin, once the values of $x$ and $y$ have been fixed to arbitrary finite values. This simple observation makes it possible to conduct calculations in the following manner: pick up suitably convenient domains for $x$ and $y$, then restrict $z$ to be such that $|z|$ is small enough; extend the result of the calculation to larger domains by virtue of unicity of analytic continuation.

Since the total number of balls at time $n$ is the deterministic quantity $s_{0}+n \sigma$, all monomials entering the trivariate GF are of the form

$$
x^{a} y^{b} z^{n}=x^{a} y^{s_{0}+n \sigma-a} z^{n}
$$

so that one has the homogeneity relation:

$$
H\left(x, y, z \mid \begin{array}{l}
a_{0} \\
b_{0}
\end{array}\right)=y^{s_{0}} H\left(x / y, 1, z y^{\sigma} \mid \begin{array}{l}
a_{0} \\
b_{0}
\end{array}\right) .
$$

From a logical standpoint, the variable $y$ is unnecessary, and we can freely set it to the value $y=1$, whenever appropriate. 
We have left aside so far the cases where $\sigma \leq 0$. When $\sigma=0$, the number of all histories satisfies

$$
H_{n}\left(\begin{array}{l}
a_{0} \\
b_{0}
\end{array}\right)=s_{0}^{n}, \quad H\left(\begin{array}{l}
z \\
a_{0} \\
b_{0}
\end{array}\right)=e^{s_{0} z} .
$$

When $\sigma=-\tau<0$ all histories are of finite length at most $s_{0} / \tau$. One has

$$
H_{n}\left(\begin{array}{l}
a_{0} \\
b_{0}
\end{array}\right)=s_{0}\left(s_{0}-\tau\right) \cdots\left(s_{0}-(n-1) \tau\right), \quad H\left(z \mid \begin{array}{l}
a_{0} \\
b_{0}
\end{array}\right)=(1+\tau z)^{\left(a_{0}+b_{0}\right) / \tau},
$$

with the trivariate GF then being a polynomial.

Before embarking in the determination of the counting generating function $H$, we note that there is an exact equivalence between the combinatorial model where all histories of a given length are considered equally likely and the original probabilistic rules governing the urn's behaviour.

Proposition 2 Let $A_{n}$ (respectively $B_{n}$ ) be the random variable representing the number of balls of colour $\times$ (respectively $\mathrm{y}$ ) of a balanced urn process at time $n$. Then, we have:

$$
\mathbb{P}\left\{A_{n}=a, B_{n}=b\right\}=\frac{\left[x^{a} y^{b} z^{n}\right] H(x, y, z)}{\left[z^{n}\right] H(1,1, z)} .
$$

In addition, at any given time, $A_{n}$ and $B_{n}$ determine one another: for an urn of balance $\sigma$, they satisfy the relation:

$$
A_{n}+B_{n}=a_{0}+b_{0}+n \sigma .
$$

Proof: This equivalence between combinatorics and probability owes to the fact that we are dealing exclusively with balanced urns (it would badly fail otherwise): the size of the urn at time $n$ is the deterministic quantity $s_{n}=s_{0}+n \sigma$, and, since histories are unambiguous descriptions of sample paths, each history of length $n$ has equal probability $\left(1 / H_{n}\right)$ of being chosen.

\subsection{The associated differential system}

Our approach relies on observing dramatically simple relations between the action of differentiation on certain expressions and the stochastic evolution of an urn. The starting point is the effect of differentiation, noted $\partial$, on monomials: the obvious relation $\partial_{x}\left[x^{n}\right]=n x^{n-1}$, can be interpreted as

$$
\partial_{x}[x x \cdots x]=(\not x x \cdots x)+(x \not x \cdots x)+\cdots+(x x \cdots \not x),
$$

meaning: "pick up in all possible ways a single occurrence of the formal variable $x$ and delete it". Similarly, the operator $x \partial_{x}$ picks up all occurrences (underlined below for readability) of the variable $x$ without deleting them:

$$
x \partial_{x}[x x \cdots x]=(\underline{x} x \cdots x)+(x \underline{x} \cdots x)+\cdots+(x x \cdots \underline{x}) .
$$

This interpretation of differentiation in terms of "marking" is classical in combinatorial analysis [9, 38, 49, 76.

Guided by the previous principles, we then associate to an urn of matrix $\mathcal{M}$ the linear partial differential operator

$$
\mathfrak{D}=x^{\alpha+1} y^{\beta} \partial_{x}+x^{\gamma} y^{\delta+1} \partial_{y},
$$

where $\partial_{x} \equiv \frac{\partial}{\partial x}$. The composition of an urn that contains at any given time $a$ balls of type $\mathrm{x}$ and $b$ balls of type y can be encoded by the monomial $\mathfrak{m}=x^{a} y^{b}$. It is easily seen that the quantity $\mathfrak{D}[\mathfrak{m}]$ generates all the possible evolution of the urn in one step:

$$
\mathfrak{D}\left[x^{a} y^{b}\right]=a x^{a+\alpha} y^{b+\beta}+b x^{a+\gamma} y^{b+\delta} .
$$

Similarly, $\mathfrak{D}^{n}\left[x^{a_{0}} y^{b_{0}}\right]$ is the generating polynomial of the configurations of the urn after $n$ steps, when its initial configuration is $\left(a_{0}, b_{0}\right)$,

$$
\mathfrak{D}^{n}\left[x^{a_{0}} y^{b_{0}}\right]=\sum_{a, b} H_{n}\left(\begin{array}{ll}
a_{0} & a \\
b_{0} & b
\end{array}\right) x^{a} y^{b}
$$

so that, using operator notation,

$$
H(x, y, z) \equiv H\left(x, y, z \mid \begin{array}{l}
a_{0} \\
b_{0}
\end{array}\right)=\sum_{n \geq 0} \mathfrak{D}^{n}\left[x^{a_{0}} y^{b_{0}}\right] \frac{z^{n}}{n !}=e^{z \mathfrak{D}}\left[x^{a_{0}} y^{a_{0}}\right] .
$$

Now, comes the central mathematical object of the present paper. 
Definition 2 Given a $2 \times 2$ urn model $\mathcal{M}$, the associated differential system is the ordinary differential system

$$
\Sigma: \quad\left\{\begin{array}{l}
\dot{x}=x^{\alpha+1} y^{\beta} \\
\dot{y}=x^{\gamma} y^{\delta+1}
\end{array}\right.
$$

There, the notation $\dot{x}$ represents differentiation with respect to the independent variable, which is by convention taken to be $t$.

The system $\Sigma$ is of a quite specific form: it is nonlinear (apart from some of the simplest cases), autonomous (meaning that independent variable $t$ does not figure explicitly), monomial (in the sense that only monomials appear in the right hand sides), and homogeneous with respect to total degree (which reflects the fact that the original urn model is balanced).

The observation that leads to our next statement is extremely simple. Let $X=X(t)$ and $Y=Y(t)$ be any particular solutions of the system (11). Consider the effect of a derivative $\partial_{t}$ on a monomial of the form $X^{a} Y^{b}$. We have:

$$
\begin{aligned}
\partial_{t}\left(X^{a} Y^{b}\right) & =a X^{a-1} \dot{X} Y^{b}+b X^{a} Y^{b-1} \dot{Y} & & \text { (by standard differentiation rules) } \\
& =a X^{a+\alpha} Y^{b+\beta}+b X^{a+\gamma} Y^{b+\delta} & & (\text { by system } \Sigma) .
\end{aligned}
$$

In other words: The effect of usual differentiation on solutions of the associated differential system $\Sigma$ mimicks the evolution of the urn $\mathcal{M}$. Accordingly, we have

$$
\partial_{t}\left(X^{a} Y^{b}\right)=\mathfrak{D}\left[x^{a} y^{b}\right] \underset{\substack{x \\ y}}{\rightarrow X Y}
$$

and upon repeating the process:

$$
\partial_{t}^{n}\left(X^{a} Y^{b}\right)=\mathfrak{D}^{n}\left[x^{a} y^{b}\right] \begin{aligned}
& x \rightarrow X \\
& y \rightarrow Y
\end{aligned}
$$

Theorem 1 (Basic isomorphism, $2 \times 2$ urns) Consider a balanced urn $\mathcal{M}$ of the form (1), (3), which is initialized with $a_{0}$ balls of type $\mathrm{x}$ and $b_{0}$ balls of type $\mathrm{y}$. Let $x_{0}, y_{0}$ be complex numbers that are each nonzero: $x_{0} y_{0} \neq 0$. Let $X\left(t \mid \begin{array}{l}x_{0} \\ y_{0}\end{array}\right)$ and $Y\left(t \mid \begin{array}{l}x_{0} \\ y_{0}\end{array}\right)$ be the solution to the associated differential system $\Sigma$ with initial conditions $x_{0}, y_{0}$. The generating function of urn histories satisfies (for $z$ small enough)

$$
H\left(x_{0}, y_{0}, z\right)=X\left(z \mid \begin{array}{l}
x_{0} \\
y_{0}
\end{array}\right)^{a_{0}} Y\left(z \mid \begin{array}{l}
x_{0} \\
y_{0}
\end{array}\right)^{b_{0}}
$$

Proof: The trivariate GF is given by 10$]$ as

$$
H(x, y, z)=\sum_{n \geq 0} \mathfrak{D}^{n}\left[x^{a_{0}} y^{b_{0}}\right] \frac{z^{n}}{n !},
$$

which by (12) and Taylor's formula yields formally

$$
H(X(t), Y(t), z)=\sum_{n \geq 0} \partial_{t}^{n}\left[X(t)^{a_{0}} Y(t)^{b_{0}}\right] \frac{z^{n}}{n !}=X(t+z)^{a_{0}} Y(t+z)^{b_{0}} .
$$

Analytically, these manipulations are justified by the Cauchy-Kovalevskaya existence theorem, which guarantees that $X(t), Y(t)$ are well defined and analytic in a neighbourhood of the origin. As a consequence, Taylor's formula can be applied and $[13$ is valid at least for some small enough values of $z$ and $t$. It then suffices to set $t=0$ to get the statement. (Observe that the condition $x_{0} y_{0} \neq 0$ is needed to ensure analyticity, in the case of negative exponents in $[11$, that is, $\alpha+1<0$ and/or $\delta+1<0$.)

Finding the trivariate $G F H(x, y, z)$ thus reduces to solving the system $\Sigma$ with "floating" initial conditions, $\left(x_{0}, y_{0}\right)$. This task is doable directly for many of the most classical urn models, for which Theorem 1 leads directly to explicit solutions, as we show in the next section (\$2). A notable feature of the argument is that it adapts to higher dimensional urn models, the ones that have balls of $m$ possible colours, for any $m \geq 3$, provided again that the models are balanced ( $\$ 9$ and Theorem 6 , p. 102, 
Note 1. The Partial Differential Equation (PDE) approach. From the exponential operator form of 10 , it immediately results that $H$ satisfies the partial differential equation:

$$
\partial_{z} H=\mathfrak{D} H, \quad \text { i.e., } \quad \frac{\partial}{\partial z} H(x, y, z)=x^{\alpha+1} y^{\beta} \frac{\partial}{\partial x} H(x, y, z)+x^{\gamma} y^{\delta+1} \frac{\partial}{\partial y} H(x, y, z) .
$$

In addition, the monomials composing $H$ satisfy a homogeneity relation: any such monomial $x^{a} y^{b} z^{n}$ with a nonzero coefficient is such that $a+b=s_{0}+n \sigma$ (see (8)), which implies

$$
\left(x \partial_{x}+y \partial_{y}-\sigma z \partial z\right) H(x, y, z)=s_{0} H(x, y, z) .
$$

Given (14) and (15), it becomes possible to eliminate the differential dependency on $y$ (that is, $\partial_{y}$ ), then set $y=1$. In this way, it is found that the bivariate generating $\bar{H}(x, z)=H(x, 1, z)$ satisfies the fundamental PDE:

$$
\left(1-\sigma z x^{\beta+\sigma}\right) \partial_{z} \bar{H}+\left(x^{\beta+\sigma+1}-x^{1-\alpha}\right) \partial_{x} \bar{H}-s_{0} x^{\beta+\sigma} \bar{H}=0 .
$$

The solution of this equation by the method of characteristics lies at the basis of the earlier study by Flajolet, Gabarró, and Pekari [30] (see also [92] for other instances), but we do not pursue this line of investigation here.

Note 2. Combinatorics of urn histories as weighted paths. An urn history can be rendered as a weighted path in the discrete Cartesian plane $\mathbb{Z} \times \mathbb{Z}$. Indeed the sequence of values of the urn's composition

$$
\left(A_{0}, B_{0}\right),\left(A_{1}, B_{1}\right), \ldots,\left(A_{k}, B_{k}\right), \ldots,\left(A_{n}, B_{n}\right),
$$

defines a polygonal line in $\mathbb{Z}^{2}$. A history is then completely specified by a sequence of integers

$$
\varpi_{0}, \varpi_{1}, \ldots, \varpi_{k}, \ldots, \varpi_{n-1},
$$

where $\varpi_{k}$ indicates which ball is picked up at time $k$ (assuming some canonical numbering that identifies balls). If $\left(A_{k}, B_{k}\right)=(x, y)$, then one should have $\omega_{k} \in[1 \ldots x+y]$, as there are $x+y$ possibilities to choose a ball. The edges connecting any two consecutive points, the "steps", are of either one of the two types,

$$
\mathbf{v}=\left(\begin{array}{c}
\alpha \\
\gamma
\end{array}\right), \quad \mathbf{w}=\left(\begin{array}{c}
\beta \\
\delta
\end{array}\right)
$$

in accordance with the rules determined by the urn's matrix $\mathcal{M}$. A v-step is chosen with a weight of $x$ (the number of possibilities for choosing an $\times$ ball), and similarly a w-step is chosen with a weight of $y$, weights being multiplicative quantities over the sequence of steps. This is summarized by the following diagram

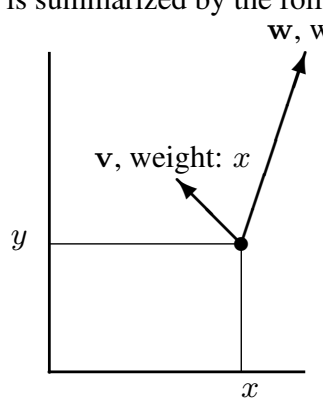

for $\mathcal{M}=[-1,3,1,1]$.

, weight: $y$

This interpretation in terms of weighted paths is well known: for instance, Kotz, Mahmoud, and Robert [74] introduce it to develop complicated multiple sum expressions for the probability distribution of the urn 's composition. Though, in all generality, the weighted path approach is ineffective for solving urn models, it is of occasional interest to combinatorialists in situations where a simple transformations can yield lattice paths of a recognizable form (see for instance [16]). This is in particular the case of the Ehrenfest urn (Note 5. p. 71), and of the OK Corral urn treated in 8. p. 99 by means of the time-reversal transformation of Kingman and Volkov [70]. Weighted lattice paths of a form similar to ours arise in Leroux and Viennot's studies of the combinatorics of nonlinear differential systems (see, e.g, [77]).

\section{Basic examples of $2 \times 2$ urns with entries in $\{0, \pm 1\}$}

Theorem 1 makes it possible to investigate directly a number of urn models, whose analyses have been previously approached by a variety of techniques, including probabilistic methods, combinatorial analysis, and the calculus of finite differences. We examine here in the light of Theorem 1 the collection of all balanced urn models whose matrices have entries in the set $\{-1,0,+1\}$. 

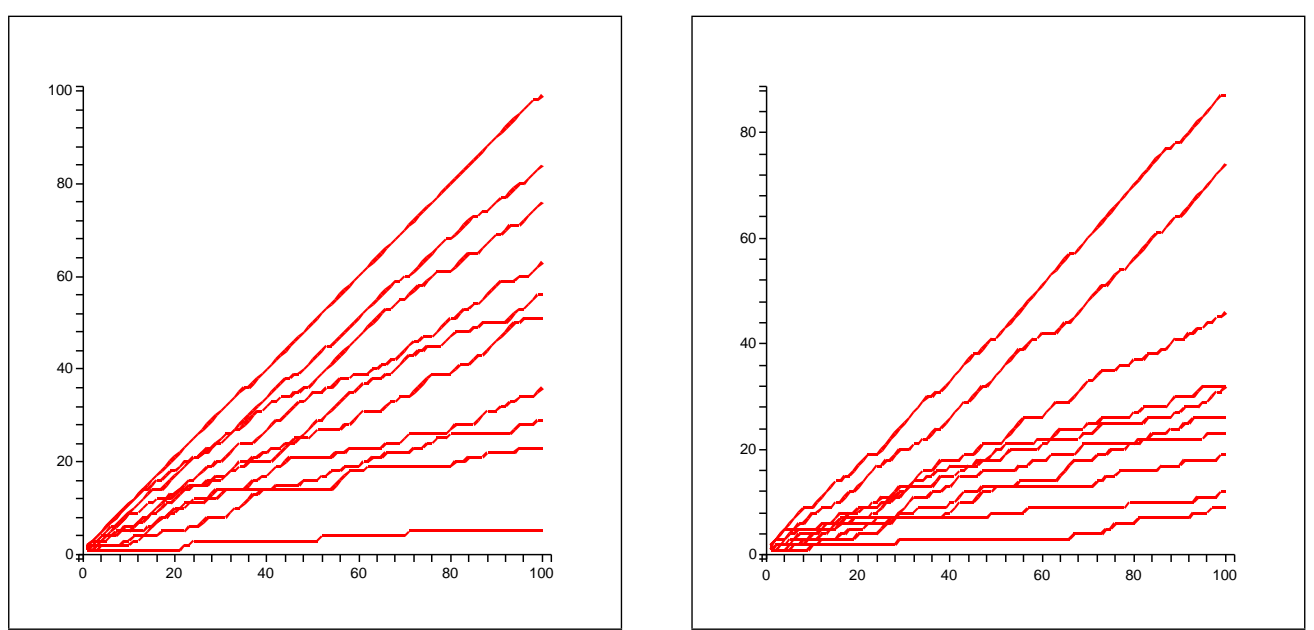

Figure 1: Ten simulations of a Pólya contagion urn over 100 steps, initialized with $(1,1)$ [left] and $(1,2)$ [right], indicate that the distribution of the urn's composition is spread. The diagrams plot the evolution of $A_{n}$ as a function of $n$.

\subsection{The contagion model (Pólya): [1, 0, 0, 1]}

The urn with matrix

$$
\mathcal{M}=\left(\begin{array}{ll}
1 & 0 \\
0 & 1
\end{array}\right)
$$

that is, the identity matrix, has a simple interpretation: one can imagine a population with two kinds of genes (or diseases) that randomly comes into contact with external elements to which their gene (or disease) is transmitted. The question is to investigate the way the initial proportion of genes of both kinds evolves with time.

The differential system is

$$
\left\{\begin{array}{l}
\dot{x}=x^{2} \\
\dot{y}=y^{2}
\end{array}, \quad\left\{\begin{array}{l}
x(0)=x_{0} \\
y(0)=y_{0}
\end{array} .\right.\right.
$$

Thanks to separation of variables, one finds $\dot{x} x^{-2}=1, \dot{y} y^{-2}=1$, which is integrated, resulting in the solution pair $(X(t), Y(t))$ :

$$
\left\{\begin{array}{l}
X(t)=\frac{x_{0}}{1-t x_{0}} \\
Y(t)=\frac{y_{0}}{1-z y_{0}}
\end{array} .\right.
$$

By Theorem 1, the complete GF of histories for this urn is

$$
H(x, y, z)=\frac{x^{a_{0}} y^{b_{0}}}{(1-z x)^{a_{0}}(1-z y)^{b_{0}}} .
$$

The urn has balance $\sigma=1$ and the number of histories of length $n$ is $H_{n}=n !\left(\begin{array}{c}n+s_{0}-1 \\ s_{0}-1\end{array}\right)$. The probability that, at time $n$, there are $a$ balls of the first type and $b$ balls of the second type (necessarily, $a+b=$ $\left.a_{0}+b_{0}+n\right)$, is obtained from the trivariate GF by coefficient extraction.

Proposition 3 For the Pólya contagion model (17), the composition of the urn at time $n$ is

$$
\mathbb{P}\left(A_{n}=a, B_{n}=b\right)=\frac{\left(\begin{array}{c}
a-1 \\
a_{0}-1
\end{array}\right)\left(\begin{array}{c}
b-1 \\
b_{0}-1
\end{array}\right)}{\left(\begin{array}{c}
a_{0}+b_{0}+n-1 \\
a_{0}+b_{0}-1
\end{array}\right)}
$$

It is not hard to establish this classical result by a direct reasoning, but the way it comes out mechanically from Theorem 1 is pleasant.

Figure 1 shows that the urn's composition at time $n$ varies widely. In fact, with $\left(a_{0}, b_{0}\right)=(1,1)$, one gets, in accordance with (18),

$$
\mathbb{P}\left(A_{n}=k\right)=\frac{1}{n+1}, \quad k=1, \ldots, n+1
$$


that is, the distribution of $A_{n}$ is uniform over the set of all possible values $[1 \ldots n+1]$, so that $A_{n} / n$ is in the limit uniform over the real interval $[0,1]$. Similarly, with $\left(a_{0}, b_{0}\right)=(1,2)$, the random variable $A_{n} / n$ has a limit distribution supported by $[0,1]$ with density $2(1-x)$, and so on. These results easily generalize to higher dimensions: see $\$ 9.1$, p. 102

\subsection{The adverse-campaign model (Friedman): $[0,1,1,0]$}

This corresponds to the antidiagonal matrix

$$
\mathcal{M}=\left(\begin{array}{ll}
0 & 1 \\
1 & 0
\end{array}\right)
$$

and it is a kind of dual to Pólya's model. This urn was studied by Friedman [43], and it can be viewed as modelling a propaganda campaign in which candidates are so bad that the persons who listen to them are convinced to vote for the opposite candidate. (This is perhaps not such an unrealistic situation!) The urn has balance $\sigma=1$. As is obvious from simulations, see Figure 2, this urn has a behaviour very different from Pólya's urn. Indeed, any large enough deviation of the urn's composition from proportions $\left(\frac{1}{2}, \frac{1}{2}\right)$ tends to correct itself and bring the system back to equilibrium, and accordingly, the composition of the urn is concentrated around its mean value.
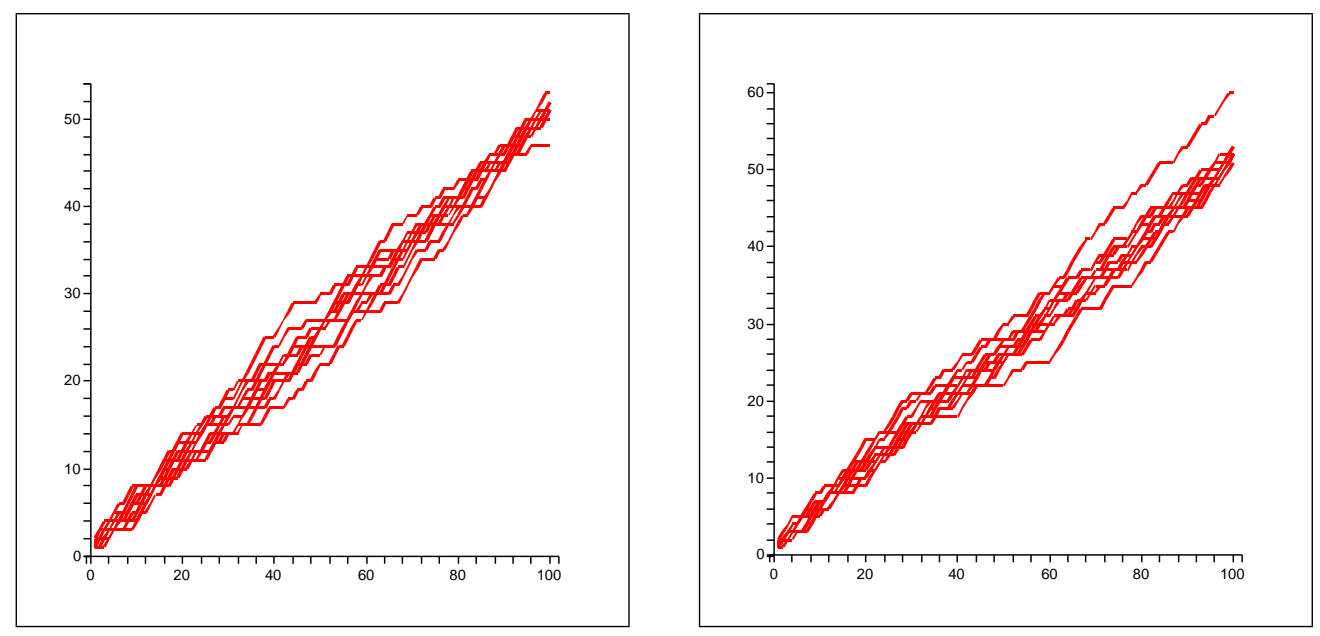

Figure 2: Ten simulations of a Friedman's urn (adverse campaign model) over 100 steps, initialized with $(1,1)$ [left] and $(1,2)$ [right], indicate that the distribution of the urn's composition is concentrated.

The associated differential system reads

$$
\left\{\begin{array}{l}
\dot{x}=x y \\
\dot{y}=x y
\end{array}, \quad\left\{\begin{array}{l}
x(0)=x_{0} \\
y(0)=y_{0}
\end{array} .\right.\right.
$$

We have $\dot{x}=\dot{y}$, so that $x$ and $y$ only differ by a constant: $y=x-x_{0}+y_{0}$. Thus, $x$ satisfies

$$
\frac{\dot{x}}{x\left(x-x_{0}+y_{0}\right)}=1
$$

which is easily integrated. The solution pair is then found to be

$$
\left\{\begin{array}{l}
X(t)=\frac{x_{0}\left(x_{0}-y_{0}\right)}{x_{0}-y_{0} e^{t\left(x_{0}-y_{0}\right)}} \\
Y(t)=\frac{y_{0}\left(y_{0}-x_{0}\right)}{y_{0}-x_{0} e^{t\left(y_{0}-x_{0}\right)}}
\end{array}\right.
$$

and the complete GF of histories is

$$
H(x, y, z)=\left(\frac{x(x-y)}{x-y e^{z(x-y)}}\right)^{a_{0}}\left(\frac{y(y-x)}{y-x e^{z(y-x)}}\right)^{b_{0}} .
$$

We single out here a simple consequence of our calculation: 


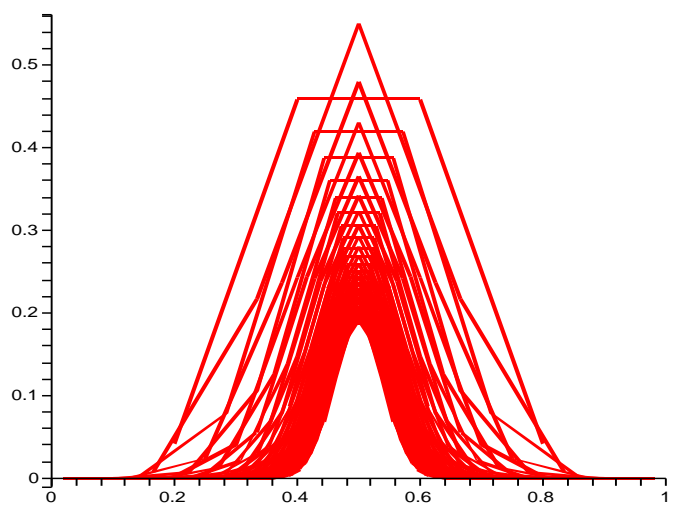

Figure 3: The histograms of the probability distribution $\mathbb{P}\left(A_{n}=k\right)$ as a function of $k /(n+1)$, for the adversecampaign urn initialized with $(1,0)$ and for $n=3 \ldots 50$, illustrate convergence to a Gaussian regime.

Proposition 4 For Friedman's adverse-campaign urn defined by (19) and for the initial condition $(1,0)$, the generating function of urn histories satisfies

$$
H(x, 1, z)=\frac{x(x-1)}{x-e^{z(x-1)}}=\frac{x(x-1)}{x-e^{z(x-1)}}=x+x z+\frac{1}{2}(x+1) x z^{2}+\frac{1}{6}\left(x^{2}+4 x+1\right) x z^{3}+\cdots
$$

In particular $n !\left[z^{n} x^{k}\right] H(x, 1, z)$ is an Eulerian number.

The bivariate generating function of Eulerian numbers is given in [15, 51]: it enumerates permutations according to their number of rises (or equivalently, descents, falls, ascents, runs).

Note 3. Acquaintance groups and the combinatorics of Friedman's urn. This urn has interesting ramifications related to the combinatorics of permutations and increasing Cayley trees, as well as to a growth model of acquaintance groups. Indeed, consider an evolving population, which at time $n$ consists of individuals numbered $\{1,2, \ldots, n+1\}$. At time $n$ any of the individuals present, say individual numbered $j$, can recruit a new member (then numbered $n+2$ ). In that case, an edge is drawn that connects $n+2$ to $j$. The collection of all edges at time $n$ constitutes a tree, which has label 1 at its root, and is such that labels along any branch stemming from the root are in increasing order-this is an increasing Cayley tree [10]. The leaves of the tree, correspond to individuals who have not recruited, call them "neophytes"; the nonleaf nodes correspond to individuals that have recruited, call them "gurus". The problem is to determine the proportion of neophytes in the group, equivalently, the number of leaves in the tree at time $n$.

Represent a neophyte by a ball of type $x=\bullet$, a guru by a ball of type $y=0$. Then, the two rules are

$$
\bullet \Longrightarrow \circ \longrightarrow \bullet, \quad \circ \Longrightarrow \text { ○—, }
$$

and it is easily recognized that it is exactly modelled by Friedman's urn, with initial conditions $(1,0)$. The solution is then provided by Equation 21. (The relation to rises in permutations can also be seen directly from existing combinatorial bijections between increasing Cayley trees, binary increasing trees, and permutations, and it is useful in the design and analysis of several classical data structures of computer science [10, 12, 38, 41, 102].) The connection with Eulerian numbers implies furthermore that the limiting distribution of balls of either kind is asymptotically normal (Figure 33, a property known for a long time in the context of run statistics in random permutations: see for instance the book by David and Barton [18 Ch. 10].

\subsection{The two-chambers urn (Ehrenfest): $[-1,1,1,-1]$}

Consider two chambers initially containing a finite number $\left(s_{0}=N\right)$ of particles. At any given time, a particle migrates from one chamber to the other (Figure 4). This famous model was first introduced by Paul and Tatiana Ehrenfest [26] in 1907 in order to investigate the way a system can reach thermodynamic equilibrium.

Colour balls according to the chamber in which they are at any given time (say $x$ for the left and $y$ for the right chamber). The system is modelled by the urn process with matrix

$$
\mathcal{M}=\left(\begin{array}{cc}
-1 & 1 \\
1 & -1
\end{array}\right)
$$




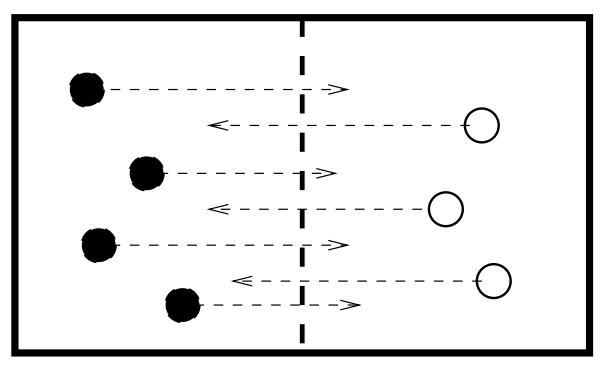

Figure 4: The Ehrenfest model (two-chambers urn).

and its balance is $\sigma=0$. The associated system is simply

$$
\left\{\begin{array}{l}
\dot{x}=y \\
\dot{y}=x
\end{array}, \quad\left\{\begin{array}{l}
x(0)=x_{0} \\
y(0)=y_{0}
\end{array}\right.\right.
$$

According to Theorem 11, it suffices to solve the system in order to determine the GF of urn histories. The derived equalities $\ddot{x}=x, \ddot{y}=y$ show that the solution is such that

$$
\left\{\begin{array}{l}
X(t)=x_{0} \cosh t+y_{0} \sinh t \\
Y(t)=x_{0} \sinh t+y_{0} \cosh t
\end{array} .\right.
$$

Thus, the trivariate GF that solves the model is

$$
H(x, y, z)=\left(\frac{x+y}{2} e^{z}+\frac{x-y}{2} e^{-z}\right)^{a_{0}}\left(\frac{x+y}{2} e^{z}-\frac{x-y}{2} e^{-z}\right)^{b_{0}} .
$$

In the standard version of the model, one starts from $a_{0}=N$ and $b_{0}=0$. In that case, the complete GF simplifies to

$$
H(x, y, z)=(x \cosh z+y \sinh z)^{N} .
$$

The total number of histories of length $n$ is $N^{n}$. The event that all particles have returned to the first chamber is then obtained by setting $x=1$ and $y=0$ in $H(x, y, z)$. Consequently:

Proposition 5 For Ehrenfest's urn defined by [22], with $N$ particles and initial configuration $(N, 0)$, the probability that, at time n, all particles have returned to the first chamber is

$$
\mathbb{P}\left(A_{N}=N, B_{n}=0\right)=\frac{n !}{N^{n}}\left[z^{n}\right] \cosh ^{N} z=\frac{1}{N^{n} 2^{N}} \sum_{k=0}^{N}\left(\begin{array}{c}
N \\
k
\end{array}\right)(N-2 k)^{n} .
$$

The formula is obtained via a binomial expansion; it is valid irrespective of the parity of $n$. The calculation is easily extended to the determination of the probability that $k$ particles have migrated (expand the result of Equation (24)).

The way the solution of this famous problem comes out automatically is noteworthy. In the past it has been approached in particular via matrix algebra, probability theory, combinatorial analysis, and continued fraction theory. A discussion of higher dimensional generalizations appears in $\$ 9.2$ p. 103.

Note 4. Matrix analysis of the Ehrenfest urn. An Ehrenfest urn can be viewed as a finite state Markov chain with $2^{N}$ states, when all balls are distinguished - it is then equivalent to a random walk on the $N$-dimensional hypercube; for $N=3$, see Figure 5 (left). States of this complete chain can be aggregated, with only the number of balls in the left chamber being retained, and this leads to a reduced Markov chain with transition probabilities represented in Figure 5 (right).

Since the Markov chain is stationary, every state is recurrent and the system will (with probability 1) return infinitely often to its initial configuration. This seems to contradict the principles of thermodynamics according to which the system should be in equilibrium, with approximately $N / 2$ balls in each chamber. The apparent contradiction is resolved by observing that statistical mechanics only represents the ideal situation of a large $N$ limit. The Ehrenfest model then serves to quantify precisely the discrepancy between a finite system and its thermodynamic limit approximation. 


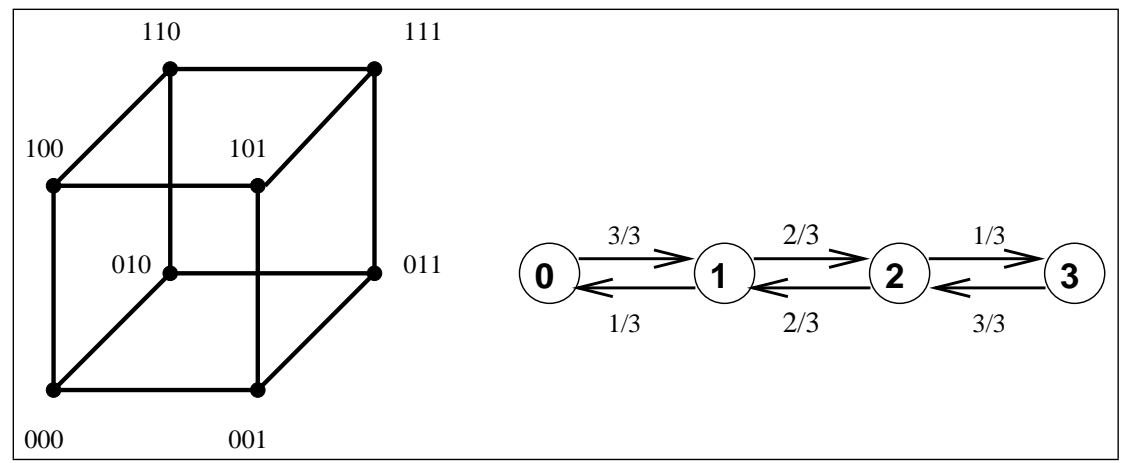

Figure 5: The complete and reduced Markov chains describing the Ehrenfest urn for $N=3$.

The analytic solution given by Marc Kac in [66] is well known. It relies on the explicit diagonalization of the $(N+1) \times(N+1)$ tridiagonal matrix of the Markov chain, which is $\frac{1}{N} \mathbf{K}$, where

$$
\mathbf{K}=\left(\begin{array}{cccccc}
0 & N & & & & \cdots \\
1 & 0 & N-1 & & & \cdots \\
& 2 & 0 & N-2 & & \cdots \\
& & 3 & 0 & N-3 & \cdots \\
\vdots & \vdots & \vdots & \vdots & \vdots & \ddots
\end{array}\right)
$$

This is a "simple matrix with many hidden treasures" in the terms of Edleman and Kostlan [25] who offer a vivid discussion, including surprising connections with random polynomials.

Note 5. Combinatorial analysis of the Ehrenfest urn. Basic combinatorics provides an attractive alternative approach to the Ehrenfest model. Consider histories of the urn that start from state $(N, 0)$ and return to state $(N, 0)$ in $n$ steps. This can be viewed as an ordered partition of the integer interval $[1 \ldots n]$ into $N$ (possibly empty) classes, where each class records the times at which a particle moved. For a particle that returns to its original chamber, its class must be of even cardinality. Then, the general theory of exponential generating functions (EGF) provides $\cosh ^{N} z$, as the EGF of all such partitions. Similarly, if $k$ out of $N$ particles migrate in the course of a history, then the EGF is directly found to be

$$
\left(\begin{array}{l}
N \\
k
\end{array}\right)(\cosh z)^{N-k}(\sinh z)^{k},
$$

which is in agreement with 24). (Proof: there are $k$ classes of odd cardinality and $N-k$ classes of even cardinality, to be chosen in $\left(\begin{array}{l}N \\ k\end{array}\right)$ ways; the odd [resp., even] classes are enumerated by $\sinh z$ [resp., $\left.\cosh z\right]$.)

An alternative derivation of the formulae relative to the Ehrenfest urn can be based on Flajolet's combinatorial theory of continued fraction [28]: histories can be transcribed as altitude-weighted Dyck paths (simple excursions) to which the theory applies. As a consequence, the ordinary generating function (OGF) of histories corresponding to a cycle involves the continued fraction

$$
\frac{1}{1-\frac{1 \cdot N z^{2}}{1-\frac{2 \cdot(N-1) z^{2}}{1-\frac{3 \cdot(N-2) z^{2}}{\ddots}}}}
$$

See Goulden-Jackson [50] for details of this approach in the case of Ehrenfest's urn and Flajolet-Guillemin [33] for an extension of the continued fraction framework to continuous-time models. (The comparison of 26) and 27, which are related by the Laplace transformation, gives then combinatorially a continued fraction first discovered analytically by Stieltjes.)

\subsection{The coupon collector's urn: $[-1,1,0,0]$}

This is the first of our models where ball colours play nonsymmetrical rôles. The urn may contain balls of either white $(x)$ or black $(y)$ colour. If a white ball is drawn, it is taken out, painted in black, then placed back into the urn; if a black ball is drawn, it is placed back unchanged into the urn. The urn's matrix is thus

$$
\left(\begin{array}{cc}
-1 & 1 \\
0 & 0
\end{array}\right)
$$


It is instantly realized that the particular initial conditions $\left(a_{0}, b_{0}\right)=(N, 0)$ model the classical coupon collector problem: a black ball represents an item that has already been drawn, a white ball an item not yet drawn. The associated differential system is

$$
\left\{\begin{array}{l}
\dot{x}=y \\
\dot{y}=y
\end{array}, \quad\left\{\begin{array}{l}
x(0)=x_{0} \\
y(0)=y_{0}
\end{array},\right.\right.
$$

and it admits the solution

$$
\left\{\begin{array}{l}
X(t)=x_{0}-y_{0}+y_{0} e^{t} \\
Y(t)=y_{0} e^{t}
\end{array}\right.
$$

Thus, the complete GF of urn histories is

$$
H(x, y, z)=\left(x-y+y e^{z}\right)^{a_{0}} y^{b_{0}} e^{b_{0} z} .
$$

The last expression specializes to give back the classical solution of the coupon collector problem. Upon setting $y=1$ and choosing the particular initial condition $(N, 0)$, one finds:

$$
H(x, 1, z)=\left(x+e^{z}-1\right)^{N} .
$$

This can be expanded:

Proposition 6 For the coupon collector's urn defined by the matrix (28) and initialized with $(N, 0)$, the probability distribution of the number of y balls at time $n$ is

$$
\mathbb{P}\left(B_{n}=b\right)=\frac{b !}{N^{n}}\left(\begin{array}{l}
N \\
b
\end{array}\right)\left\{\begin{array}{l}
n \\
b
\end{array}\right\},
$$

where $\left\{\begin{array}{l}n \\ b\end{array}\right\}$ is a Stirling number of the partition type or of "second kind".

See [15, 51] for the usual definition of Stirling numbers. Quite remarkably, this urn model already appears explicitly in Laplace's Théorie analytique des probabilités [75], first published around 1811—Laplace indeed assigns the original question to [Jacob] Bernoulli. Gani [44] has recently made use of this urn to model certain infectious phenomena (the propagation of diseases by means of infected syringes).

\subsection{The sampling urns: $[0,0,0,0]$ and $[-1,0,0,-1]$}

The urn with the null matrix

$$
\left(\begin{array}{ll}
0 & 0 \\
0 & 0
\end{array}\right)
$$

models sampling with replacement. We are initially given $a_{0}$ white (x) balls and $b_{0}$ black (y) balls. A ball drawn is placed back into the urn, irrespective of its colour, so that the composition of the urn never changes. Thus the evolution is nothing but a succession of independent Bernoulli trials (with respective probabilities $a_{0} / s_{0}$ and $\left.b_{0} / s_{0}\right)$. The system is $\dot{x}=x, \dot{y}=y$, so that $X(t)=x_{0} e^{t}$ and $Y(t)=y_{0} e^{t}$. The complete GF is accordingly

$$
H(x, y, z)=x^{a_{0}} y^{b_{0}} e^{\left(a_{0}+b_{0}\right) z} .
$$

(The binomial law arises when one counts the number of times a white balls has been drawn.)

The urn with matrix

$$
\left(\begin{array}{cc}
-1 & 0 \\
0 & -1
\end{array}\right)
$$

corresponds to sampling without replacement and is more interesting. The system is

$$
\left\{\begin{array}{l}
\dot{x}=1 \\
\dot{y}=1
\end{array}, \quad\left\{\begin{array}{l}
x(0)=x_{0} \\
y(0)=y_{0}
\end{array} .\right.\right.
$$

The complete GF of urn histories is then

$$
H(x, y, z)=(x+z)^{a_{0}}(y+z)^{b_{0}},
$$

from which the probability distribution of $A_{n}, B_{n}$ reads off:

$$
\mathbb{P}\left(A_{n}=a, B_{n}=b\right)=\frac{\left(\begin{array}{c}
a_{0} \\
a
\end{array}\right)\left(\begin{array}{c}
b_{0} \\
b
\end{array}\right)}{\left(\begin{array}{c}
a_{0}+b_{0} \\
a+b
\end{array}\right)},
$$

with $a+b=n$. This is again extremely classical. 


\subsection{The records urn $[1,0,1,0]$}

This urn is formed with identical rows, so that the composition of the urn at time $n$ is entirely deterministic:

$$
A_{n}=a_{0}+n, \quad B_{n}=b_{0} .
$$

From the associated differential system, we find mechanically:

$$
H(x, y, z)=\frac{x^{a_{0}} y^{b_{0}}}{(1-z x y)^{a_{0}+b_{0}}} .
$$

Though the composition of the urn at various instants is predictable, the number of times a ball of either type is chosen in the course of an evolution of length $n$ is of interest.

Let the colours $x$ and $y$ be named white and black, respectively, and start with a single black ball, so that $a_{0}=0, b_{0}=1$. At time $n$, there are $n$ white balls and exactly one black ball. The probability that the black ball is picked up is $1 /(n+1)$. Thus, the number $S_{n}$ of times that the black ball is chosen in the course of an $n$ step evolution is a sum of independent Bernoulli random variables:

$$
S_{n}=X_{1}+X_{2}+\cdots+X_{n}, \quad X_{j} \in \operatorname{Bern}\left(\frac{1}{j}, 1-\frac{1}{j}\right) .
$$

This random variable also represents the number of records in a random permutation of size $n+1$. (A direct proof results from the classical construction of inversion tables [15].) For this special case, the differential equation approach to urn models can be adapted as follows. It suffices to introduce a variable $w$ that keeps track of the choices of a black ball and define the (modified) differential system:

The solution is

$$
\left\{\begin{array}{l}
\dot{x}=x^{2} \\
\dot{y}=w x y
\end{array}, \quad\left\{\begin{array}{l}
x(0)=x_{0} \\
y(0)=y_{0}
\end{array} .\right.\right.
$$

$$
X(t)=\frac{x_{0}}{1-x_{0} t}, \quad Y(t)=y_{0}\left(1-x_{0} t\right)^{-w} .
$$

The variables $x_{0}, y_{0}$ are redundant and we may freely set them to 1 . For $\left(a_{0}, b_{0}\right)=(0,1)$, the outcome is the bivariate generating function of the number of records (and cycles) in a permutation, which comes out as

$$
\frac{1}{(1-z)^{w}}
$$

a classical result for the generating function of Stirling cycle numbers [15, 51]. (This urn is also closely related to what Pitman names the "Chinese Restaurant Process" [89] and to the Ewens sampling distribution of population genetics [54].)

\subsection{The all-ones urn: $[1,1,1,1]$}

Like the previous one, this urn has identical rows, so that the composition of the urn at time $n$ is deterministic. The associated differential system is

$$
\left\{\begin{array}{l}
\dot{x}=x^{2} y \\
\dot{y}=x y^{2}
\end{array}, \quad\left\{\begin{array}{l}
x(0)=x_{0} \\
y(0)=y_{0}
\end{array} .\right.\right.
$$

The solution is easily determined, once we notice that $\dot{x} x^{-1}=\dot{y} y^{-1}$, which implies that $x$ and $y$ are proportional and $y / y_{0}=x / x_{0}$. We find

$$
X(t)=\frac{x_{0}}{\sqrt{1-2 x_{0} y_{0} t}}, \quad Y(t)=\frac{y_{0}}{\sqrt{1-2 x_{0} y_{0} t}},
$$

so that the complete GF of urn histories is

$$
H(x, y, z)=\frac{x^{a_{0}} y^{b_{0}}}{(1-2 z x y)^{\left(a_{0}+b_{0}\right) / 2}} .
$$

Like for the records urn, our methods extend to a characterization of the number of times a ball of each type is chosen. The end result is similar to the Stirling distribution of the Records Urn.

This concludes the discussion of all the ten cases of balanced urns whose matrices have entries restricted to $\{-1,0,+1\}$, once we take into account the equivalence between urns

$$
\left(\begin{array}{ll}
\alpha & \beta \\
\gamma & \delta
\end{array}\right) \cong\left(\begin{array}{cc}
\delta & \gamma \\
\beta & \alpha
\end{array}\right)
$$

corresponding to an interchange of colours $x$ and $y$. 


\section{The first integral of balanced $2 \times 2$ urns}

It turns out that urn models of dimension two (i.e., with two types of balls) that are balanced can always be solved in finite terms. This results from the existence of a first integral for the associated differential system. An essential parameter of the urn is the dissimilarity index of the urn model or matrix, which we define by

$$
p:=\gamma-\alpha=\beta-\delta
$$

(The equivalence of the two forms requires the balance condition.) The dissimilarity parameter measures the differential benefit, seen from either ball, resulting from the colour of the ball chosen. It is then natural to consider the following three categories.

- Altruistic urns correspond to $p>0$. In that case, a ball chosen "gives" to the population of balls of the other colour.

- Neutral urns correspond to $p=0$. In that case, the composition of the urn at any instant $n$ is entirely determined (and is a linear function of time). Such models are of moderate interest since they can be studied directly like in the case of the records urn and the all-ones urn of the previous section. Accordingly, we need not discuss them any further.

- Selfish urns correspond to $p<0$. In that case a ball chosen serves its own kind more than the other kind. In a sense, it reinforces its own population.

The family of all $2 \times 2$ balanced urn matrices is a three parameter family. The quantities $\sigma$ (the sum) and $p$ (the dissimilarity) are two parameters, which, together with any single entry of the matrix, determine the model. We also observe that the matrix $\mathcal{M}$ admits $\sigma$ as an eigenvalue, with right eigenvector the column vector $(1,1)^{\mathrm{T}}$. The other eigenvalue is $-p=\alpha-\gamma$ with left eigenvector $(1,-1)$.

Note 6. The spectrum of the urn's matrix. In the classical theory of urn models, eigenvalues play an important rôle, since they determine to a large extent the urn's probabilistic behaviour. For a balanced $2 \times 2$ urn with $\sigma>0$, it has been recognized for a long time that the ratio of eigenvalues,

$$
\rho:=-\frac{p}{\sigma} \equiv \frac{\alpha-\gamma}{\alpha+\beta}
$$

is the basic discriminating quantity. One has a priori $\rho \leq 1$, since $\rho>0$ implies $\alpha>0$ and, in turn, $(\alpha-\gamma) /(\alpha+\beta) \leq$ $\alpha /(\alpha+\beta) \leq 1$. Gouet [47] uses martingale arguments to prove functional limit theorems to the effect that under the condition,

$$
\rho \leq \frac{1}{2}
$$

(together with $\beta \gamma \neq 0$, i.e., for nontriangular cases), the evolution of the urn is Gaussian-this includes all sacrificial urns discussed in $\$ 5$ and $\$ 6$ for which one has $\rho<0$. (Several such results do extend to urns of higher dimensions as described in 9 , see for instance [62, 96].)

Finally, an urn model is said to be arithmetically irreducible if the four matrix entries have no common divisor:

$$
\operatorname{gcd}(\alpha, \beta, \gamma, \delta)=1
$$

With little loss of generality, we shall normally confine ourselves to the study of such arithmetically irreducible urns. Roughly, if $d>1$ is a common divisor of all matrix entries, it is possible to group balls by packs of $d$ and operate with the reduced matrix that has entries $[\alpha / d, \beta / d, \gamma / d, \delta / d]$. (The only price to be paid is a minor restriction on initial conditions, namely $d \mid a_{0}$ and $d \mid b_{0}$, but our methods can easily be extended to cover such situations.)

Proposition 7 The differential system,

$$
\left\{\begin{array}{l}
\dot{x}=x^{\alpha+1} y^{\beta} \\
\dot{y}=x^{\gamma} y^{\delta+1}
\end{array}, \quad \alpha+\beta=\gamma+\delta\right.
$$

admits $x^{p}-y^{p}$ as a first integral, where $p=\beta-\alpha=\gamma-\delta$ is the dissimilarity parameter. Precisely, let

$$
X(t)=X\left(t \mid \begin{array}{l}
x_{0} \\
y_{0}
\end{array}\right), \quad Y(t)=Y\left(t \mid \begin{array}{l}
x_{0} \\
y_{0}
\end{array}\right),
$$

be the solution of the system corresponding to the initial conditions $X(0)=x_{0}$ and $Y(0)=y_{0}$, with $x_{0} y_{0} \neq 0$. Then, for $t$ in some complex neighbourhood of 0 , one has

$$
X(t)^{p}-Y(t)^{p}=x_{0}^{p}-y_{0}^{p} .
$$


Proof: It suffices to compute

$$
\frac{d}{d t}\left(x^{p}-y^{p}\right)=p x^{p-1} \dot{x}-p y^{p-1} \dot{y}=p x^{\alpha+p} y^{\beta}-p x^{\gamma} y^{\delta+p} \equiv 0,
$$

which ensures that $x^{p}-y^{p}$ is a constant.

In other words, the solutions $X(t), Y(t)$ to the differential system provide a local parameterization of a curve of Fermat type,

$$
X^{p}-Y^{p}=\text { Constant } .
$$

In a sense, this explains that the two-chambers urn (Ehrenfest urn), which has $p=2$, gives rise to a solution involving hyperbolic functions, which parametrize the hyperbola $Y^{2}-X^{2}=1$. The contagion urn (Pólya urn) has $p=-1$, so that $X^{-1}-Y^{-1}=C$ (with $C$ a constant), and it is accordingly described by linear fractional transformations.

\section{General solutions for $2 \times 2$ sacrificial urns $(\alpha \leq-1)$}

Equipped with the first integral of Proposition 7, we proceed to solve the associated differential system. Several cases are to be distinguished based, inter alia, on the sign of $p$. In this section, we operate with the assumption

$$
\alpha \leq-1
$$

implying $p>0$, thus corresponding to an upper diagonal entry that is negative and implying an urn with positive dissimilarity. Balls of colour $x$ are said to be sacrificial since, whenever drawn, a certain number of them is withdrawn. An urn with at least one negative diagonal entry is said to be sacrificial. It is said to be semi-sacrificial, respectively fully sacrificial, if the number of its negative diagonal entries is exactly 1 , respectively 2 .

We start by restricting $y_{0}$ in a neighbourhood of 1 and $x_{0}$ in a neighbourhood of 0 . Eventually, for the probabilistic analysis, we shall set $y_{0}=1$ (since the information provided by the variable $y$ in the complete GF is redundant and tolerates setting $y=1$ ) and take $x_{0}$ to vary in domains containing 0 . We make use of the following notations,

$$
\Delta\left(x_{0}, y_{0}\right):=\left(y_{0}^{p}-x_{0}^{p}\right)^{1 / p}, \quad \Delta\left(x_{0}\right):=\left(1-x_{0}^{p}\right)^{1 / p},
$$

with principal determinations of $p$ th roots intended when $x_{0} \approx 0, y_{0} \approx 1$. So far, we have only made use of the property $p>0$, a consequence of (31).

Our goal is to eliminate the "floating" initial conditions and introduce a standard set of functions by which one can completely express the complete GF of urn histories. Given the first integral of Proposition 7, one can eliminate $y$ in the equation defining $x$ :

$$
\dot{x}=x^{\alpha+1} y^{\beta}=x^{\alpha+1}\left(x^{p}-x_{0}^{p}+y_{0}^{p}\right)^{\beta / p}=x^{\alpha+1}\left(x^{p}+\Delta^{p}\right)^{\beta / p},
$$

and similarly for $y$. This can be reduced to a form that no longer involves "floating" initial conditions. Set

$$
X\left(t \mid \begin{array}{l}
x_{0} \\
y_{0}
\end{array}\right)=\Delta \xi\left(t \Delta^{\sigma}\right), \quad Y\left(t \mid \begin{array}{l}
x_{0} \\
y_{0}
\end{array}\right)=\Delta \eta\left(t \Delta^{\sigma}\right) .
$$

Then, the transformed system satisfied by the pair $(\xi, \eta)$ is

$$
\left\{\begin{array}{l}
\dot{\xi}=\xi^{\alpha+1}\left(\xi^{p}+1\right)^{\beta / p} \\
\dot{\eta}=\eta^{\delta+1}\left(\eta^{p}-1\right)^{\gamma / p}
\end{array}, \quad\left\{\begin{array}{l}
\xi(0)=x_{0} \Delta^{-1} \\
\eta(0)=y_{0} \Delta^{-1}
\end{array} .\right.\right.
$$

In particular, there is separation of variables,

$$
\frac{\dot{\xi}}{\xi^{\alpha+1}\left(1+\xi^{p}\right)^{\beta / p}}=1
$$

so that on can solve for $\xi$ :

$$
t=\int_{x_{0} / \Delta}^{\xi(t)} \frac{w^{-\alpha-1}}{\left(1+w^{p}\right)^{\beta / p}} d w
$$




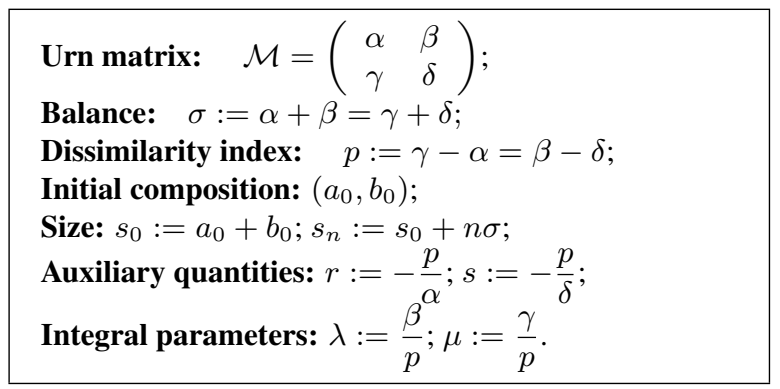

Figure 6: A summary of notations for 2-colour urn models.

This defines the $\xi$ function by inversion of an Abelian integra (iii) Hence the solution of the associated system and the complete GF of histories are expressible by means of an Abelian integral over the Fermat curve,

$$
\mathbf{F}_{p}: \quad Y^{p}-X^{p}=1,
$$

and its inverse functions (with respect to composition).

At this stage, we make use of the assumption $\alpha+1 \leq 0$ in (31). This means that balls of the $x$ colour "sacrifice" themselves in favour of balls of colour y. A change of variable $w^{-\alpha}=\zeta$ in the integral of (35) first reduces the definition of $\xi$ to (remember that $-\alpha$ is a positive number)

$$
t=-\frac{1}{\alpha} \int_{\left(x_{0} / \Delta\right)^{-\alpha}}^{\xi(t)^{-\alpha}} \frac{d \zeta}{\left(1+\zeta^{r}\right)^{\beta / p}}, \quad r:=-\frac{p}{\alpha} .
$$

Note that $r$ is a positive integer, given the tenability conditions which require $-\alpha \mid \gamma$. The desired standardization is provided by the following definition.

Definition 3 Let the parameters $r$ and $\lambda$ satisfy $r \in \mathbb{Z}_{>0}$ and $\lambda \in \mathbb{Q}_{\geq 0}$. The fundamental integral $J=J_{r, \lambda}$ is defined to be

$$
J(u):=\int_{0}^{u} \frac{d \zeta}{\left(1+\zeta^{r}\right)^{\lambda}} .
$$

The pseudo-sine function $S=S_{r, \lambda}$ is defined in a complex neighbourhood of 0 as the inverse of J:

$$
S(J(u))=J(S(u))=u .
$$

The pseudo-cosine function $C=C_{r, s, \lambda}$ is defined in a complex neighbourhood of 0 as

$$
C(z)=\left(1+S(z)^{r}\right)^{1 / s}
$$

where the principal determination of the sth root is adopted.

We note that, near $0, J(u)$ is analytic and satisfies $J(u)=u+O\left(u^{r+1}\right)$, so that it is analytically invertible near 0 . Thus, the function $S$ is analytic at 0 and satisfies $S(z)=z+O\left(z^{r+1}\right)$. The function $C(z)$ is also analytic and satisfies $C(z)=1+O\left(z^{r}\right)$. Precisely:

$$
\left\{\begin{aligned}
J(u) & =u-\frac{\lambda}{r+1} u^{r+1}+\frac{\lambda(\lambda+1)}{2(2 r+1)} u^{2 r+1}-\frac{\lambda(\lambda+1)(\lambda+2)}{6(3 r+1)} u^{3 r+1}+\cdots \\
S(z) & =z+\frac{\lambda}{r+1} z^{r+1}+O\left(z^{2 r+1}\right) \\
C(z) & =1+\frac{1}{s} z^{r}+O\left(z^{2 r}\right) .
\end{aligned}\right.
$$

(By its very definition, $J(u)$ only includes powers of the form $\left\{u^{k r+1}\right\}_{k \geq 0}$. As a consequence, $S(z)$ and $C(z)$ only include terms of the form $\left\{z^{k r+1}\right\}_{k \geq 0}$ and $\left\{z^{k r}\right\}_{k \geq 0}$, respectively.)

The reason for the chosen denomination is that the functions $S, C$ can be thought of as higher degree analogues of trigonometric or hyperbolic functions. (In fact, minor variants of these functions have been studied in the nineteenth century by Lundberg [81] under the name of hypergoniometric functions, their

\footnotetext{
(iii) Given an algebraic curve defined by the polynomial equation $P(x, y)=0$, an Abelian integral is any integral of the form $\int Q(x, y) d x$, with $Q$ a rational function.
} 
study having been recently revitalized by Lindqvist and Peetre [78, 79].) In particular, when $p=r=2$ and $\lambda=1 / 2$, the functions $J, S, C$ reduce to

$$
J(u)=\int_{0}^{u} \frac{d \zeta}{\sqrt{1+\zeta^{2}}}=\operatorname{arcsinh}(u), \quad S(z)=\sinh (z), \quad C(z)=\cosh (z),
$$

which is how they appear in the two-chambers (Ehrenfest) urn model.

We can now state:

Theorem 2 Consider a balanced urn model whose matrix $\mathcal{M}=\left(\begin{array}{ll}\alpha & \beta \\ \gamma & \delta\end{array}\right)$ has $\alpha \leq-1$ and (iv) $\delta \neq 0$. Then the bivariate generating function of urn histories, for $(x, z)$ in a neighbourhood of $(0,0)$ is given by the analytic expression:

$$
\begin{aligned}
H(x, 1, z)= & \Delta^{s_{0}} S\left(-\alpha z \Delta^{\sigma}+J\left(x^{-\alpha} \Delta^{\alpha}\right)\right)^{-\frac{a_{0}}{\alpha}} C\left(-\alpha z \Delta^{\sigma}+J\left(x^{-\alpha} \Delta^{\alpha}\right)\right)^{-\frac{b_{0}}{\delta}} \\
& \text { with } \Delta \equiv \Delta(x)=\left(1-x^{p}\right)^{1 / p} .
\end{aligned}
$$

There, the usual notations are used,

$$
\sigma=\alpha+\beta, \quad p=\beta-\alpha, \quad s_{0}=a_{0}+b_{0},
$$

and we have $J=J_{r, \lambda}$ (the fundamental integral of (37)), $S=J^{(-1)}$ defined by $S(J(u))=J(S(u))=u$ (the pseudo-sine of (38)), $C$ defined by $C(z)=\left(1+S(z)^{r}\right)^{1 / s}$ (the pseudo-cosine of (39)), the parameters being

$$
p:=\gamma-\alpha, \quad \lambda=\frac{p}{\beta}, \quad r:=-\frac{p}{\alpha}, \quad s:=-\frac{p}{\delta},
$$

so that $\lambda \in \mathbb{Q}_{\geq 0}, r \in \mathbb{Z}_{\geq 1}$, and $s \in \mathbb{Q}$.

Proof: We have from 36:

$$
-\alpha t=J\left(\xi(t)^{-\alpha}\right)-J\left(x_{0}^{-\alpha} \Delta^{\alpha}\right) .
$$

This relation can be inverted to express $\xi^{-\alpha}$ :

$$
\xi^{-\alpha}=S\left(-\alpha t+J\left(x_{0}^{-\alpha} \Delta^{\alpha}\right)\right),
$$

and hence $X(t)^{-\alpha}$, by Equation (33). Observe that, though $\xi$ and $X$ are not in general analytic at $0, \xi^{-\alpha}$ and $X^{-\alpha}$ are. The function $\eta^{-\delta}$ is similarly expressed in terms of $C$, since $\eta^{p}-\xi^{p}=1$. The statement finally results from plugging the explicit forms of $X, Y$ into the formulæ provided by Theorem 1 , upon effecting the substitution $x_{0} \rightarrow x, y_{0} \rightarrow 1$. The expression obtained is bivariate analytic in a neighbourhood of $(0,0)$, as it should, owing to the tenability conditions, which ensure that the exponent of the pseudo-sine, namely $-a_{0} / \alpha$, is necessarily an integer.

We present now in Examples 14 several examples that illustrate the variety of special functions that may appear in $2 \times 2$ urn models.

Example 1. The two-chambers urn (Ehrenfest), $[-1,1,1,-1]$, revisited. This urn justifies our basic terminology regarding pseudo-trigonometric functions. The matrix is

$$
\left(\begin{array}{cc}
-1 & 1 \\
1 & -1
\end{array}\right)
$$

so that we have

The fundamental integral is

$$
\sigma=0, \quad \alpha=-1, \quad p=r=2, \quad \beta=1 .
$$

$$
J(u)=J_{2,1 / 2}(u)=\int_{0}^{u} \frac{d \zeta}{\sqrt{1+\zeta^{2}}}=\operatorname{arcsinh}(u) .
$$

Consequently, the pseudo-sine and pseudo-cosine are

$$
S(z)=S_{2,1 / 2}(z)=\sinh (z), \quad C(z)=C_{2,1 / 2}(z)=\cosh (z) .
$$

A mechanical application of Theorem 2 then provides, when $s_{0}=a_{0}=N\left(\right.$ and $\left.b_{0}=0\right)$ :

$$
H(x, 1, z)=\left(1-x^{2}\right)^{N / 2} \sinh ^{N}\left(z+\operatorname{arcsinh}\left(\frac{x}{\sqrt{1-x^{2}}}\right)\right),
$$

which is easily seen to be equivalent to the result of [24, given basic trigonometric identities.

(iv) The case $\delta=0$ corresponds to "antitriangular" urns and needs minor adjustments in definitions: see Example 2 below. 
Example 2. The peaks-in-perms urn (Mahmoud): $[-1,2,1,0]$. This urn has matrix

$$
\left(\begin{array}{cc}
-1 & 2 \\
1 & 0
\end{array}\right)
$$

and parameters

$$
\sigma=1, \quad p=r=2, \quad \lambda=\frac{1}{2} .
$$

The fundamental integral is accordingly

$$
J(u)=J_{2,1}(u)=\int_{0}^{u} \frac{d \zeta}{1+\zeta^{2}}=\arctan (u),
$$

so that the pseudo-trigonometric functions are usual ones:

$$
S(z)=\tan (z), \quad \check{C}(z)=\sqrt{1+\tan ^{2}(z)}=\sec (z)=\frac{1}{\cos (z)} .
$$

In this case, we have $\delta=0$, so that $s=\infty$, which forces us to adopt, instead of $C$, the function $\check{C}$ satisfying $\check{C}^{r}-S^{r}=1$. Then the bivariate GF of urn histories is for $a_{0}=1$ and $b_{0}=0$ :

$$
H(x, 1, z)=\Delta(x) \tan \left(z \Delta(x)+\arctan \left(\frac{x}{\Delta(x)}\right)\right), \quad \Delta(x):=\sqrt{1-x^{2}} .
$$

an expression that can be expanded to yield

$$
H(x, 1, z)=x+z+2 x \frac{z^{2}}{2 !}+\left(2+4 x^{2}\right) \frac{z^{3}}{3 !}+\left(16 x+8 x^{3}\right) \frac{z^{4}}{4 !}+\left(16+88 x^{2}+16 x^{4}\right) \frac{z^{5}}{5 !}+\cdots
$$

Note that, if we set $x=0$, we get the GF of alternating (or up-and-down or zigzag) permutations [15, pp. 258-259].

We recognize in the last expansion a variant of the sequence EIS:A008303 in Sloane's Encyclopedia of Integer Sequences [95], where it is described as "Permutations of $[n]$ with $k$ peaks". Indeed, values in a permutation $\sigma=$ $\left(\sigma_{1}, \ldots, \sigma_{n}\right)$ can be classified into four types known as peaks $\left(\sigma_{i-1}<\sigma_{i}>\sigma_{i+1}\right)$, valleys aka troughs $\left(\sigma_{i-1}>\sigma_{i}<\right.$ $\left.\sigma_{i+1}\right)$, double rises $\left(\sigma_{i-1}<\sigma_{i}<\sigma_{i+1}\right)$, and double falls $\left(\sigma_{i-1}>\sigma_{i}>\sigma_{i+1}\right)$. See for instance the books of David and Barton [18 Ch. 10] or Stanley [97] p. 24], as well as the article by Françon and Viennot [42]. A well known GF due to Carlitz enumerates permutations according to the number of elements of each type, and $H$ in 42 is a special case. In fact, in an article dedicated to patterns in permutations and increasing binary trees ${ }^{(v)}$ the authors of [32, p. 238] derived an expression essentially equivalent to that of 42 and deduced a local limit law of the Gaussian type for the coefficients.

The correspondence between this urn model and increasing binary trees results from observations made by Mahmoud in the related context of binary search trees: Mahmoud explicitly introduced this urn in his review paper [84] and our description is essentially an adaptation of his. First, it is well known that increasing binary trees under the uniform model can be obtained by a simple growth model: view external nodes (that are unlabelled) as "placeholders"; start from a single external node; at each discrete instant $n+\frac{1}{2}$, pick up uniformly at random such an external node, and attach to it an internal node labelled $n+1$ with two dangling external nodes. In the standard bijection between permutations and increasing binary trees [10, 38, 97], an element that is neither a peak nor a valley corresponds to a one-way branching internal node, hence to an external node whose brother is an internal node; a leaf (an internal node attached to two external nodes) corresponds to a peak. It is then possible to model the growth of an increasing binary tree by an urn model, while at the same time keeping track of peaks: Encode by a white ball $(x)$ an external node whose brother is an internal node; encode by a black ball (y) an external node whose brother is an external node. It is then easily seen that the inductive construction of the tree corresponds to the urn process with matrix $[-1,2,1,0]$. The correspondence between this urn model and peaks in permutation is thus established.

Example 3. The first algebraic urn: $[-1,3,1,1]$. This urn has matrix and parameters given by

$$
\mathcal{M}=\left(\begin{array}{cc}
-1 & 3 \\
1 & 1
\end{array}\right), \quad \sigma=2, \quad p=r=2, \quad \lambda=\frac{3}{2} .
$$

Its interest is to admit a complete GF that is an algebraic function of low degree.

The fundamental integral is explicit:

$$
J(u) \equiv J_{2,3 / 2}(u)=\int_{0}^{u} \frac{d \zeta}{\left(1+\zeta^{2}\right)^{3 / 2}}=\frac{u}{\sqrt{1+u^{2}}} .
$$

\footnotetext{
(v) An increasing binary tree is a plane binary tree, which has internal nodes labelled by distinct integers and is such that labels along any branch stemming from the root go in increasing order. An increasing binary tree is in bijective correspondence with a permutation of the same size_-simply read off the labels in infix order. See [97 p. 24] and [10] for instance. The model is equivalent to that of random binary search trees [94].
} 
Then inversion provides for the pseudo-sine and pseudo-cosine:

$$
S(z)=\frac{z}{\sqrt{1-z^{2}}}, \quad C(z)=\sqrt{1-z^{2}} .
$$

Say we consider initial configurations with $\left(a_{0}, b_{0}\right)=(0, N)$. The complete GF of urn histories is found to be

$$
H(x, 1, z)=\left(1-x^{2}\right)^{N / 2} \frac{1}{\left(1-\left(z\left(1-x^{2}\right)+x\right)^{2}\right)^{N / 2}} .
$$

Such an expression can be used to determine explicitly moments of the number of balls of the first colour. Take for instance $N=1$. It suffices to expand locally near $x=1$ :

$$
H(x, 1, z)=\frac{1}{\sqrt{1-2 z}}+(x-1) \frac{z(1-z)}{(1-2 z)^{3 / 2}}+\frac{(x-1)^{2}}{2} \frac{z^{2}\left(2-4 z+3 z^{2}\right)}{(1-2 z)^{5 / 2}}+O\left((x-1)^{2}\right.
$$

Each partial derivative $\left.\partial_{x}^{r} H(x, 1, z)\right|_{x=1}$ is a simple radical function, and in particular, the total number of histories is (as anticipated)

$$
H_{n}=n ! \cdot\left[z^{n}\right] \frac{1}{\sqrt{1-2 z}}=2^{n} n !\left(\begin{array}{c}
n-\frac{1}{2} \\
n
\end{array}\right)=1 \cdot 3 \cdot 5 \cdots(2 n-1) .
$$

As a consequence: Each moment of the number of balls of a given colour is a rational function of $n$. Asymptotically, we find easily (with the Help of Dr Salvy's programme equivalent, under the symbolic manipulation system MAPLE):

$$
\mathbb{E}\left(A_{n}\right)=\frac{1}{2} n+\frac{1}{4}+\frac{1}{8 n}+O\left(\frac{1}{n^{2}}\right), \quad \mathbb{V}\left(A_{n}\right)=\frac{1}{4} n+\frac{1}{8}+\frac{1}{8 n}+O\left(\frac{1}{n^{2}}\right),
$$

where $\mathbb{E}$ and $\mathbb{V}$ represent expectation and variance, respectively, and $A_{n}$ is as usual the number of balls of colour $\mathrm{x}$ present at time $n$. Thus the distribution is concentrated around its mean and there is convergence in probability:

$$
\frac{A_{n}}{n / 2} \stackrel{P}{\longrightarrow} 1
$$

We shall see in $\$ 6$ that such closed form formulæ and simple asymptotic forms for moments are the rule. Their derivation in the present context is almost immediate, this in sharp contrast with the fact they are often obtained in the literature at the expense of costly recurrence manipulations $s^{(\text {vi) }}$

The probability of all balls being of the second colour at time $n=2 \nu$ also results from the expression of $H$. It is obtained by setting $x=0$ in $H(x, 1, z)$,

so that, upon extracting coefficients:

$$
H(0,1, z)=\frac{1}{\sqrt{1-z^{2}}}
$$

$$
\mathbb{P}\left(A_{2 \nu}=0\right)=\frac{(2 \nu) !^{3}}{\nu !^{2}(4 \nu) !} \sim 2^{-2 \nu+1 / 2} .
$$

Like in the case of the generation-parity urn, the probability of such an extreme deviation is well quantified and turns out to be exponentially small. (See $\$ 5$ for a general theory of such urns and $\$ 5.3$ for a class generalizing the present example.)

Example 4. The generation-parity model: $[-1,2,2,-1]$. Consider the urn with matrix

$$
\left(\begin{array}{cc}
-1 & 2 \\
2 & -1
\end{array}\right)
$$

and parameters

$$
\sigma=1, \quad p=r=3, \quad \lambda=\frac{2}{3} .
$$

This urn models a binary splitting process as follows. There are two types of particles, $\mathrm{x}$ and $\mathrm{y}$. At a discrete instant, any of the particles present in the system has equal chances of disintegrating, with an $x$ particle giving rise to two $y$ particles and a y particle giving rise to two $x$ particles. Assume for simplicity that one starts at time 0 with one particle of type $\mathrm{x}$, so that $a_{0}=1, b_{0}=0$. If particles are numbered according to the value of $n$ determining the time $\left(n+\frac{1}{2}\right)$ at which they disintegrate, the process is once more represented by an increasing binary tree. Balls of colour $x$ correspond

\footnotetext{
(vi) Our point of view is that traditional recurrence manipulations in the case of balanced urns at least, are normally subsumed by more synthetic generating function methods. This observation is perhaps to be somewhat nuanced, and for instance the fundamental paper of Bagchi and Pal [6] has to be singled out: by a shrewd use of recurrence techniques, the authors of [6] were first able to deduce the asymptotic form of centred moments of the urn's composition and deduce a Gaussian limiting distribution for a large class of balanced urns. However, as we shall see later, the global structure of all moments can be unwound for large classes of urns. An elegant point of view on moment calculations is developed by Pouyanne in [91, 90].
} 
to balls that belong to an even generation (as measured by the distance to the root node); balls of colour y belong to a generation which is odd.

The fundamental integral is

$$
J(u)=J_{3,2 / 3}(u)=\int_{0}^{u} \frac{d \zeta}{\left(1+\zeta^{3}\right)^{2 / 3}},
$$

which is an Abelian over the Fermat curve $\mathbf{F}_{3}: Y^{3}-X^{3}=1$. The inverse function has been studied by Alfred Cardew Dixon (1865-1936) in a long article [20] and further examined by Conrad and Flajolet (vii) in [16] as regards combinatorial properties. What is of special interest is the fact that $\mathrm{smh}:=S$ and its companion $\mathrm{cmh}:=C$ are elliptic functions (i.e., meromorphic doubly periodic functions [13,103]). With

$$
\begin{aligned}
\operatorname{smh}(z) & :=S_{3,2 / 3}(z)=\operatorname{Inv} \int_{0}^{z} \frac{d \zeta}{\left(1+\zeta^{3}\right)^{2 / 3}} \\
& =z+4 \frac{z^{4}}{4 !}+160 \frac{z^{7}}{7 !}+20800 \frac{z^{10}}{10 !}+6476800 \frac{z^{13}}{13 !}+\cdots,
\end{aligned}
$$

the inverse function (viii) of $J(u)$ (our notation follows [16, 20]), Theorem 2 provides the complete GF of histories (Proposition 3 in [16]):

$$
\begin{aligned}
H(x, 1, z) & =\left(1-x^{3}\right)^{1 / 3} \operatorname{smh}\left(\left(1-x^{3}\right)^{1 / 3} z+\int_{0}^{x\left(1-x^{3}\right)^{-1 / 3}} \frac{d s}{\left(1+s^{3}\right)^{2 / 3}}\right), \\
& =x+\frac{z}{1 !}+2 x^{2} \frac{z^{2}}{2 !}+\left(4 x^{3}+2 x^{4}\right) \frac{z^{3}}{3 !}+\cdots .
\end{aligned}
$$

The function smh, which has positive coefficients, has a dominant singularity on the positive real axis, by virtue of Pringsheim's Theorem. Since $J(u)$ is analytic at any finite positive point $u>0$, where it satisfies $J^{\prime}(u) \neq 0$, it is invertible. Thus, the dominant singularity $\rho>0$ of smh along the positive real axis can only be obtained with $\operatorname{smh}(\rho)=\infty$, that is, we must have

$$
\rho=J(\infty)=\frac{1}{2 \pi \sqrt{3}} \Gamma\left(\frac{1}{3}\right)^{3},
$$

where the latter evaluation results from the Eulerian Beta integral [103].

Here is a nice application of (43), (44), and (45). Say we want to estimate the probability that at time $n$, all balls be of the second colour, that is, all particles alive in the system belong to some odd numbered generation. This probability is obtained by considering $H(0,1, z)$, so that $\mathbb{P}\left(A_{n}=0\right)=\left[z^{n}\right] \operatorname{smh}(z)$. (This can only happen with nonzero probability at instants of the form $n=3 \nu+1$.) Then, by an easy analysis of singularities, we find

$$
\mathbb{P}\left(A_{3 \nu+1}=0\right) \sim c \rho^{-3 \nu-1}, \quad \rho=\frac{1}{2 \pi \sqrt{3}} \Gamma\left(\frac{1}{3}\right)^{3} .
$$

For instance, we find to 10 decimal places (10D):

$$
\left(\frac{\left[z^{28}\right] \operatorname{smh}(z)}{\left[z^{31}\right] \operatorname{smh}(z)}\right)^{1 / 3} \doteq 1.7666387490 \cdots . \quad \rho \doteq 1.7666387502 \cdots
$$

Equation 46 thus quantifies accurately an extreme large deviation regime that has an exponentially small probability of occurrence. (See $\$ 6$ for a general theory of such urns and, in particular, 6.2 for further elliptic connections.)

Note 7. The pseudo-cosine as an inverse Abelian integral. Coming back to the transformed system (34), we have

$$
\frac{\dot{\eta}}{\eta^{\delta+1}\left(\eta^{p}-1\right)^{\gamma / p}}=1
$$

This implies (compare with Equation (35)

$$
t=\int_{y_{0} / \Delta}^{\eta(t)} \frac{w^{-\delta-1}}{\left(w^{p}-1\right)^{\gamma / p}}
$$

and via a change of variables similar to 36

$$
t=-\delta \int_{\left(y_{0} / \Delta\right)^{-\delta}}^{\xi^{-\delta}} \frac{d \zeta}{\left(\zeta^{s}-1\right)^{\mu}}, \quad s=-\frac{p}{\delta}, \quad \mu=\frac{\gamma}{p} .
$$

Thus: the pseudo-cosine is alternatively definable as the inverse of an Abelian integral.

\footnotetext{
(vii) Regarding the genesis of the present paper, an important starting point for the differential system approach to urn models is the detailed study of the combinatorics of Dixonian elliptic functions in [16].

(viii) The coefficients constitute the sequence EIS:A104133.
} 
Note 8. Hypergeometric form of the fundamental integrals. The hypergeometric function $F={ }_{2} F_{1}$ is classically defined [103] as

$$
{ }_{2} F_{1}\left[\begin{array}{c|c}
a, b & z \\
c & z
\end{array}\right]=1+\frac{a \cdot b}{c} \frac{z}{1 !}+\frac{a(a+1) \cdot b(b+1)}{c(c+1)} \frac{z^{2}}{2 !}+\cdots .
$$

The fundamental integral (37) is then a special hypergeometric function,

$$
J_{r, \lambda}(u)=u \cdot F\left[\begin{array}{c|c}
\lambda, 1 / r & -u^{r} \\
1+1 / r
\end{array}\right]
$$

as is seen from expanding and integrating term-by-term. Thus, with Inv representing inversion of functions with respect to composition, one has for an urn with matrix $[\alpha, \beta ; \gamma, \delta]$ :

$$
S(z)=\operatorname{Inv}\left(z \cdot F\left[\begin{array}{c|c}
\beta / p,-\alpha / p \\
1-\alpha / p
\end{array} \mid-z^{r}\right]\right) .
$$

Thus: the pseudo-sine function is the compositional inverse of a special hypergeometric function. A similar property holds for the pseudo-cosine. We shall not however make much use of hypergeometric function theory in the present paper.

\section{The class of $2 \times 2$ semi-sacrificial urns $(\alpha \leq-1, \delta>0)$}

Recall that a colour is sacrificial if the corresponding diagonal entry in its matrix $\mathcal{M}$ is negative. The last section has started a general treatment of the case $\alpha<0$, that is, colour $\mathrm{x}$ is sacrificial. Starting from there, two subclasses of urns may be considered: $(i)$ the fully sacrificial urn models for which $\alpha<0$ and $\delta<0$; (ii) the semi-sacrificial urns for which $\alpha<0$ and $\delta>0$. The former case has been treated fairly thoroughly in the earlier article of Flajolet, Gabarró and Pekari [30] and is reviewed in the next section. The latter case is the one of interest for us now. In summary, our present object of study is the class of matrices (ix)

$$
\mathcal{M}=\left(\begin{array}{cc}
\alpha & \beta \\
\gamma & \delta
\end{array}\right), \quad \alpha<0, \quad \delta>0, \quad \beta \gamma \neq 0, \quad \sigma>0
$$

What we want to do is exploit the analytic consequences of Theorem 2 in a way dictated by the need of asymptotic probability. As we shall see, the complex geometry of the problem is quite different from that of [30]. A sufficient fragment of this geometry is worked out in the next subsection. After this, routine calculations make it possible to derive precise estimates relative to extreme deviations, a Gaussian limit law (with speed of convergence estimates), the large deviation rate function, and the structure of moments.

\subsection{The analytic structure of the base functions $S, C$}

By the term of base functions, we mean here the two functions

$$
S(z), \quad C(z)
$$

which serve to express $H(x, 1, z)$, the bivariate generating function (BGF) of urn histories. As is usual in analytic combinatorics, singularities are essential. The first fundamental result is the following.

Lemma 1 Let $\mathcal{M}$ be a semi-sacrificial urn model in the sense of the conditions (47).

(i) The base functions $S(z)$ and $C(z)$ are analytic near 0 . Their common radius of convergence $\rho$ is given by $(p=\gamma-\alpha, r=-p / \alpha, s=-p / \delta)$

$$
\rho=J(+\infty)=\frac{1}{r} B\left(\frac{1}{r}, 1-\frac{1}{r}-\frac{1}{s}\right)=\frac{1}{r} \frac{\Gamma\left(\frac{1}{r}\right) \Gamma\left(1-\frac{1}{r}-\frac{1}{s}\right)}{\Gamma\left(\frac{1}{s}\right)} .
$$

(ii) The only dominant singularities (i.e., the singularities of smallest modulus) of $S(z)$ and $C(z)$ are at the conjugate points

$$
\rho \omega^{j}, \quad \text { for } j=0,1, \ldots, r-1, \quad \text { where } \omega=e^{2 i \pi / r} \text {. }
$$

Proof: The proof consists in establishing four properties: $(A)$ the radius of convergence is a singularity and its form is explicit; $(B) S(z)^{r}$ avoids the value $(-1)$ inside its disc of convergence; $(C)|S(z)|$ remains bounded as $z$ tends radially to a boundary point that is not a singularity; $(D)$ the function $S(z)$ is analytic at end points $\rho e^{i \theta}$ except for a collection of $r$ special directions, as described in the statement.

\footnotetext{
(ix) Triangular urns corresponding to $\beta \gamma=0$ will be dealt with in separate sections.
} 
(A) Consider the BGF of histories $H(x, 1, z)$ for an initial composition of the urn given by $\left(a_{0}, b_{0}\right)=$ $(-\alpha, 0)$. Then, by Theorem 2 , we have

$$
H(0,1, z)=S(-\alpha z)
$$

This is the exponential GF of urn histories conditioned upon the fact that no ball of colour $\mathrm{x}$ is present in the end. The equation shows that $S(-\alpha z)$, hence $S(z)$, has nonnegative Taylor coefficients.

By Pringsheim's theorem [38, 99], since $S(z)$ has nonnegative coefficients, the set of all its dominant singularities includes the value of the radius of convergence $\rho$ of the series at 0 . In particular, in order to determine that radius, it suffices to examine the function $S$ along the ray $\mathbb{R}_{\geq 0}$, starting from 0 , till a singularity is encountered.

The main point is that $S$ is defined as the inverse of $J$ and $J(u)$ is analytic at all points of the positive real axis. At the same time, for any $u \in \mathbb{R}_{\geq 0}$, one has $J^{\prime}(u) \equiv\left(1+u^{r}\right)^{-\lambda} \neq 0$. There results that $J$ is an analytically invertible mapping of the interval $[0,+\infty[$ onto $[0, \rho[$, where $\rho=J(+\infty)$. The latter quantity is nothing but an Eulerian Beta integral whose evaluation in terms of $\Gamma$ factors is classical [103]. These considerations justify the first assertion $(i)$ of the lemma for $S(z)$.

$(B)$ We next prove that for all $z$ such that $|z|<\rho$, we have $S(z)^{r} \neq-1$. Observe that $S(z)$ is a solution of the differential equation

$$
Y^{\prime}(z)=\left(1+Y(z)^{r}\right)^{\lambda},
$$

with $\lambda>1$, given the conditions 477 . Now, it is easy to verify that a solution $Y(z)$ cannot be analytic in the neighbourhood of a point $z_{0}$ such that $Y\left(z_{0}\right)^{r}=-1$. Indeed, if $Y(z)$ were analytic, we would have

$$
Y(z) \sim \eta+c \eta\left(z-z_{0}\right)^{a}, \quad Y^{\prime}(z) \sim \operatorname{ca\eta }\left(z-z_{0}\right)^{a-1}, \quad\left(1+Y(z)^{r}\right)^{\lambda} \sim(-r c)^{\lambda}\left(z-z_{0}\right)^{\lambda a},
$$

for some $a \geq 1, \eta^{r}=-1$, and some $c \neq 0$. The equality $Y^{\prime}=\left(1+Y^{r}\right)^{\lambda}$ is clearly impossible since $\lambda=\beta /(\beta-\delta)>1$, so that the orders of growth of $Y^{\prime}$ and $\left(1+Y^{r}\right)^{\lambda}$ are incompatible.

This avoidance property implies that $C(z)$ is analytic in the disc $|z|<\rho$. A simple computation also shows that it is singular as $z \rightarrow \rho^{-}$. Thus, part $(i)$ of the statement has been established for $C(z)$.

Next, we observe, from the nature of the expansions of $S$ and $C$ at 0 that these functions have a rotational symmetry, namely

$$
S(\omega z)=\omega S(z), \quad C(z)=C(\omega z) .
$$

Thus, it is sufficient to study $S(z), C(z)$ in any sector rooted at 0 with opening angle $2 \pi / r$. Also, this symmetry immediately implies that the conjugate quantities $\rho \omega^{j}$ are amongst the dominant singularities of $S$ and $C$. It takes however a bit of work to establish that there are no dominant singularities besides these.

$(C)$ We now proceed to investigate the behaviour of $S(z)$ along rays of angle $\theta$, with $0<\theta<\pi / r$, which is sufficient by rotational symmetry and conjugacy. Define the semi-open interval

$$
\mathcal{I}[\theta]:=\left\{x e^{i \theta} \mid 0 \leq x<\rho\right\} .
$$

The proof of boundedness properties of $S$ makes use of the differential relations satisfied by $S$. Choose first an arbitrary $R \in(0, \rho)$. The "daffodil lemma" of [38] expresses the fact that the directions of largest increase of the modulus of an analytic function with nonnegative coefficients at 0 are to be found amongst a set of regularly spaced rays emanating from 0 ; furthermore these directions are determined by the period (or span) of the support of the coefficients of $f$ (i.e., the set of $n$ such that $f_{n} \neq 0$ ). Here this lemma implies that, for $R \in(0, \rho)$ and for any $\theta$ with $0<\theta<\pi / r$, we have $\left|S\left(R e^{i \theta}\right)\right|<S(R)$. In particular, there exists an $x_{0}<R$ such that $\left|S\left(R e^{i \theta}\right)\right|=S\left(x_{0}\right)$. We can then find an $x_{1}$ in $\left(x_{0}, R\right)$, such that

$$
\left|S\left(R e^{i \theta}\right)\right|<S\left(x_{1}\right) .
$$

We then propose to compare for $x \in[R, \rho[$, the two functions

$$
Y(x):=S\left(x e^{i \theta}\right), \quad Z(x):=S\left(x_{1}+x-R\right),
$$

which satisfy the autonomous differential equations

$$
\left\{\begin{array}{l}
Y^{\prime}(x)=\left(1+Y(x)^{r}\right)^{\lambda} e^{i \theta} \\
Y(R)=S\left(R e^{i \theta}\right)
\end{array}, \quad\left\{\begin{array}{l}
Z^{\prime}(x)=\left(1+Z(x)^{r}\right)^{\lambda} \\
Z(R)=S\left(x_{1}\right)
\end{array}\right.\right.
$$


Roughly, we can visualize the values of $Y, Z$ as the trajectory of two mobiles whose movement is governed by similar differential equations. In terms of modulus, i.e., distance to the origin, $Y$ is the tortoise and $Z$ the hare, but contrary to what happens in the fable, the tortoise never catches up since it starts late and its speed remains dominated by that of the hare (see [17] for another context). Consequently, till time $x=\rho$, the function $Y(z)$ remains of modulus smaller than $Z(\rho)=S\left(x_{1}-R+\rho\right)<+\infty$. The argument is made precise by writing the differential systems in integral form:

$$
\left\{\begin{array}{l}
Y(x)=Y(R)+\int_{R}^{x}\left(1+Y(\xi)^{r}\right)^{\lambda} e^{i \theta} d \xi \\
Z(x)=Z(R)+\int_{R}^{x}\left(1+Z(\xi)^{r}\right)^{\lambda} d \xi
\end{array}\right.
$$

(Note that the determination of the $\lambda$ th root is well defined by continuity since $S(z)^{r}$ avoids -1 .) Assume a contrario that there exists an $\bar{x}$ with $\bar{x}<R+\rho-x_{1}$ such that $|Y(\bar{x})|=Z(\bar{x})$, and let $x_{2}$ be the smallest such $\bar{x}$. We would then have, by trivial inequalities,

$$
\begin{aligned}
\left|Y\left(x_{2}\right)\right| & \leq|Y(R)|+\int_{R}^{x_{2}}\left(1+|Y(\xi)|^{r}\right)^{\lambda} d \xi \\
& <Z(R)+\int_{R}^{x_{2}}\left(1+Y(\xi)^{r}\right)^{\lambda} d \xi=Z\left(x_{2}\right)
\end{aligned}
$$

where the second inequality is strict since $|Y(x)|<Z(x)$ at least for a small interval to the right of $R$. A contradiction with the supposition that $\left|Y\left(x_{2}\right)\right|=Z\left(x_{2}\right)$ has been reached. In other words, we have proved that $S(z)$ remains of bounded modulus along $\mathcal{I}[\theta]$.

$(D)$ We now establish that the end point $\rho e^{i \theta}$ of $\mathcal{I}[\theta]$ is such that $S(z)$ is analytic there. Say that a direction $\theta$ different from the singular directions of the statement is proper if the set of limit values of $S\left(x e^{i \theta}\right)^{r}$ as $x \rightarrow \rho$ includes at least one element that is different from $(-1)$.

Consider first a proper direction, with $0<\theta<\pi / r$, and let $v_{0}$ be any limit value of $S(z)$ along $I[\theta]$ satisfying $v_{0}^{r} \neq-1$. By classical existence theorems for ordinary differential equations, the equation

$$
\Upsilon^{\prime}(z)=\left(1+\Upsilon(z)^{r}\right)^{\lambda}, \quad \Upsilon^{\prime}(0)=v,
$$

admits a solution $\Upsilon(z)=\Upsilon(z, v)$ that is bivariate analytic in a neighbourhood $\Omega \subset \mathbb{C}^{2}$ of $\left(0, v_{0}\right)$, which we can fix to be the polydisc

$$
|z|<\delta, \quad\left|v-v_{0}\right|<\epsilon .
$$

Choose then $z_{0}=\rho_{0} e^{i \theta}$ such that

$$
\left|\rho e^{i \theta}-\rho_{0} e^{i \theta}\right|<\frac{\delta}{2}, \quad\left|v-v_{0}\right|<\epsilon
$$

Then, we must have

$$
S(z)=\Upsilon\left(z-z_{0}, S\left(z_{0}\right)\right)
$$

As a consequence of this equality, $S(z)$ is itself analytic at $\rho e^{i \theta}$. Thus, along any proper direction, $S(z)$ is analytically continuable beyond the boundary of its disc of convergence.

There only remains to exclude improper directions. Assume a contrario that there exists an improper direction $\theta$. Then, by definition of properness, $S(z)^{r}$ admits -1 as unique limit value. In addition, we claim that there exists a particular $r$ th $\operatorname{root} \eta$ of -1 such that $S(z)$ tends to the limit $\eta$ as $z \rightarrow \rho e^{i \theta}$. (Proof: otherwise, $S$, which is bounded, would oscillate between several roots of -1 , but, then, its set of limit values would include quantities other than the $r$ th roots of -1 , a contradiction with the improperness assumption.) Supposing still $\theta$ to be an improper direction, let $\gamma$ be the image curve by $S$ of the open ray $\mathcal{I}[\theta]$. We have

$$
J^{\gamma}(S(z))=z \quad \text { for all } z=x e^{i \theta}, \text { with } 0 \leq x<\rho,
$$

where $J^{\gamma}(u)$ means that the integral defining $J$ is to be taken along the path $\gamma$. (Proof: the identity is true for $z$ near 0 and it extends to the whole of $\gamma$ by analytic continuation.) But then, we reach a contradiction since, all determinations of $J$ are such that $J(\eta)$ is infinite (the integral defining $J$ diverges), while (51) implies that $J$ must remain finite. Thus, no improper direction exists.

We have thus shown that all points of the disc of radius $\rho$ other than $\rho$ and its conjugate are regular points for $S$. We have also shown that the value -1 is not approached by $S(z)^{r}$, as $z$ tends to the boundary of 
the disc of radius $\rho$, so that the stated analyticity properties of $S$ in the closed disc of radius $\rho$ also hold for $C(z)$. The proof of the lemma is now complete.

The next step consists in characterizing the singular expansions of $S(z)$ and $C(z)$.

Lemma 2 Let $\sigma>0$. In the complex plane slit along the ray $\mathbb{R}_{\geq \rho}$, one has

$$
S(z) \underset{z \rightarrow \rho}{=}\left(r_{0}(\rho-z)\right)^{-1 / r_{0}}\left[1-\frac{\lambda}{r_{1}}\left(r_{0}(\rho-z)\right)^{r / r_{0}}+O\left((\rho-z)^{2 r / r_{0}}\right)\right],
$$

where $r_{0}=r \lambda-1, r_{1}=r(\lambda+1)-1$. The conjugate expansions at the points $\rho \omega^{j}$ of (49) are determined by the fact that $S(z) / z$ is an analytic function of $z^{r}$ at 0 . Similarly for $C(z)$ :

$$
C(z) \underset{z \rightarrow \rho}{=}\left(r_{0}(\rho-z)\right)^{-r /\left(s r_{0}\right)}\left[1+\frac{1}{s}\left(1-\frac{\lambda r}{r_{1}}\right)\left(r_{0}(\rho-z)\right)^{r / r_{0}}+O\left((\rho-z)^{2 r / r_{0}}\right)\right],
$$

with the conjugate expansions being determined by the fact that $C(z)$ is an analytic function of $z^{r}$ at 0 .

Proof: This is a simple exercise in computational asymptotics. The expansion of $J(u)$ as $u \rightarrow+\infty$ results from the expansion of the integrand $\left(1+\zeta^{r}\right)^{-\lambda}$ :

$$
J(u) \underset{u \rightarrow+\infty}{=} \rho-\frac{1}{r \lambda-1} \frac{1}{u^{r \lambda-1}}+\frac{1}{r(\lambda+1)-1} \frac{1}{u^{r(\lambda+1)-1}}+O\left(\frac{1}{u^{r(\lambda+2)-1}}\right) .
$$

Thus, $\rho-J(u)$ is of the order of $1 / u^{r_{0}}$, which, given that $J$ and $S$ are inverse pair, means that $\rho-z$ is of the order of $1 / S(z)^{r_{0}}$ as $z \rightarrow \rho$. The main term of $S(z)$ follows; further terms are obtained by series reversion.

This formal process provides the singular expansion of $S(z)$ in the form

$$
\left.\left(r_{0}(\rho-z)\right)^{-1 / r_{0}} A\left(r_{0}(\rho-z)\right)^{r / r_{0}}\right)
$$

with $A(w)$ a formal power series in $w$ with $A(0)=1$. Adopting $\tau=\left(r_{0}(\rho-z)\right)^{r / r_{0}}$ as uniformizing parameter and making use of the fact that the compositional inverse of an analytic function is analytic (provided, as is the case here, that the first derivative does not vanish), we see that $A(w)$ is furthermore analytic at 0 . The validity of the expansion in the slit plane results.

\subsection{Probabilistic properties of semi-sacrificial urns}

Equipped with a good understanding of the analytic structure of the basic functions $S$ and $C$, which involve the single complex variable $z$, we can now proceed with the analysis of the bivariate generating function $H(x, 1, z)$. The treatment follows the customary lines exposed in the book Analytic Combinatorics [38] and they parallel what was done earlier in [30] in the case of fully sacrificial urn models. We state here in plain words a collection of probabilistic properties whose detailed formulations constitute Propositions 8 11 below.

Theorem 3 (Semi-sacrificial urns) Consider a semi-sacrificial urn determined by a matrix satisfying the conditions of (47).

( $i)$ The probability that, at time $n$, all balls are of the second type $\left(A_{n}=0\right)$ is exponentially small; the exponential rate is a quotient of Gamma function values taken at rational arguments.

(ii) The number of balls of the first type $\left(A_{n}\right)$ converges in distribution, in the large $n$ limit, to a normal. The speed of convergence to the Gaussian limit is a negative fractional power of $n$.

(iii) The number $A_{n}$ of balls of the first type satisfies the mean and variance estimates: $\mathbb{E}\left(A_{n}\right) \sim \mu n$ and $\mathbb{V}\left(A_{n}\right) \sim \xi^{2} n$, for computable constants $\mu, \xi$; in particular, $A_{n} / n$ converges in probability to $\mu$. Generally, any rth moment admits a closed-form expression, which is of hypergeometric type.

(iv) The number $A_{n}$ of balls of the first type satisfies a large deviation principle, with the large deviation rate function being effectively computable from the fundamental integral $J$ of the urn model.

The extreme large deviation result result $(i)$ and its companion $(i v)$ are, to the best of our knowledge, new; technically, they closely parallel the case of fully sacrificial urns discussed in [30]. The Gaussian limit law of $(i i)$ is known from works of Bachi, Pal, and Smythe [6, 96], but the characterization of the speed of convergence seems to be new. 


\subsubsection{Extreme large deviations}

Like before, we let $\left(A_{n}, B_{n}\right)$ be the vector describing the urn's composition at time $n$ : there are $A_{n}$ balls of type $\mathrm{x}$ and $B_{n}$ balls of type $\mathrm{y}$. For a semi-sacrificial urn, balls of type y never disappear, so that $B_{n}>0$ for all $n \geq 1$. A boundary configuration is the one in which $A_{n}=0$, corresponding to an extreme in the large deviation regime (this terminology follows [30]). We state:

Proposition 8 The probability of an extreme large deviation, corresponding to the event $A_{n}=0$, is exponentially small. One has for large $n$ satisfying $n \equiv-\frac{a_{0}}{\alpha} \bmod r$ :

$$
\mathbb{P}\left(A_{n}=0\right)=C R^{n} n^{\kappa}\left(1+O\left(n^{-e}\right)\right), \quad e=\min \left(1, \frac{\beta-\alpha}{\beta+\alpha}\right),
$$

where $C>0$ and

$$
\kappa=s_{0}\left(\frac{1}{p}-\frac{1}{\sigma}\right), \quad R=\left(\frac{-\alpha}{\alpha+\beta}\right) \rho^{-1}, \quad \rho \equiv \frac{1}{r} \frac{\Gamma\left(\frac{1}{r}\right) \Gamma\left(1-\frac{1}{r}-\frac{1}{s}\right)}{\Gamma\left(\frac{1}{s}\right)} .
$$

Proof: The number of configurations is obtained by setting $x=0$ in the expression (41) of the BGF (bivariate generating function) $H(x, 1, z)$. We have, by Theorem 2 .

$$
H(0,1, z)=S(-\alpha z)^{-a_{0} / \alpha} C(-\alpha z)^{-b_{0} / \delta}
$$

if the urn is initialized with $\left(a_{0}, b_{0}\right)$. Consideration of the supports of $S(z)$ and $C(z)$ shows that $A_{n}=0$ can only happen at instants equal to $-a_{0} / \alpha$ plus a multiple of $r$, a fact which may otherwise be confirmed by a combinatorial reasoning.

The statement then results from singularity analysis [34, 38] applied to the BGF $H(0,1, z)$, as given by (55), and a trite calculation.

\subsubsection{A Gaussian limit law with speed}

The knowledge of the bivariate generating function of urn histories combined with the characterization of singularities of the fundamental functions $S, C$ gives access to the asymptotic law of the urn's composition $\left(A_{n}, B_{n}\right)$ at every instant. We start with a purely analytic lemma describing the radius of convergence of urn histories.

Lemma 3 Let $\rho(x)$ be the radius of convergence of the bivariate generating function of urn histories $H(x, 1, z)$ considered as a function of $z$. There exists a complex neighbourhood of $x=1$ in which this radius is an analytic function of $x$ and is given by the formula

$$
\rho(x)=x^{-\sigma} \sum_{k \geq 0}\left(\begin{array}{c}
-\lambda \\
k
\end{array}\right) \frac{1}{\sigma+k p}\left(\frac{1-x^{p}}{x^{p}}\right)^{k} .
$$

Proof: The radius of convergence $\rho(x)$ is determined by

$$
-\alpha \rho(x) \Delta(x)^{\sigma}+J\left(x^{-\alpha} \Delta(x)^{\alpha}\right)=\rho,
$$

where $\rho=J(+\infty)$ is the common radius of convergence of $S(z)$ and $C(z)$. Define the quantity

$$
\mathfrak{e}(x)=\frac{\Delta(x)}{x}=\frac{\left(1-x^{p}\right)^{1 / p}}{x} .
$$

The quantity $\mathfrak{e}(x)^{\alpha}$ becomes infinite as $\mathfrak{e}(x) \rightarrow 0$, that is as $x \rightarrow 1$, since $\alpha \leq-1$ by assumption. By comparing (57) for general $x$ and for $x=1$, we find

$$
\mathfrak{e}^{-\sigma}\left(\rho-J\left(\mathfrak{e}^{\alpha}\right)\right)=\mathfrak{e}^{-\sigma} \int_{\mathfrak{e}^{\alpha}}^{\infty} \frac{d \zeta}{\left(1+\zeta^{r}\right)^{\lambda}} .
$$

The change of variables $\zeta=v^{\alpha}$ followed by term-by-term integration of the series expansion of the integrand then yields the stated form of $\rho(x)$. 
Next, from the general expression of $H(x, 1, z)$ and from the singular expansions of $S(z)$ and $C(z)$, we see directly that

$$
H(x, 1, z) \underset{z \rightarrow \rho(x)}{\sim} \sigma^{-s_{0} / \sigma}(\rho(x)-z)^{-s_{0} / \sigma} .
$$

In other words, the function $H(x, 1, z)$, regarded as a function of $z$ with $x$ a parameter, has a movable singularity and a constant exponent. In particular, for an $x$ suitably close to 1 , one has

$$
\left[z^{n}\right] H(x, 1, z) \sim \frac{\sigma^{-s_{0} / \sigma}}{\Gamma\left(s_{0} / \sigma\right)} \rho(x)^{-n-s_{0} / \sigma} n^{s_{0} / \sigma-1},
$$

by virtue of singularity analysis theory [34,38]. These estimates are seen to be consistent with the case $x=$ 1 , which corresponds to the plain enumeration of urn histories.

A trite calculation suffices to enhance (58): one finds

$$
\begin{aligned}
H(x, 1, z) \underset{z \rightarrow \rho(x)}{=} \frac{1}{(\sigma(\rho(x)-z))^{s_{0} / \sigma}[1} & +\frac{\gamma b_{0}-\beta a_{0}}{p(p+\sigma)}\left(1-x^{p}\right)(\sigma(\rho(x)-z))^{p / \sigma} \\
& \left.+O\left((\rho(x)-z)^{2 p / \sigma}\right)\right]
\end{aligned}
$$

where the error term is uniform with respect to $x$ in a sufficiently small neighbourhood of 1 . It is known that singularity analysis preserves the uniform character of expansions with respect to a parameter (see comments in [34] and [38, Ch. IX]). As a consequence, one has

$$
\left[z^{n}\right] H(x, 1, z) \underset{n \rightarrow \infty}{=} \frac{\sigma^{-s_{0} / \sigma}}{\Gamma\left(s_{0} / \sigma\right)} \rho(x)^{-n-s_{0} / \sigma} n^{s_{0} / \sigma-1}\left[1+O\left(n^{-p / \sigma}\right)\right],
$$

uniformly for $x$ sufficiently close to 1 . The expression simplifies further, when one considers the probability generating function of $A_{n}$. One has

$$
\chi_{n}(x):=\mathbb{E}\left(x^{A_{n}}\right) \equiv \frac{\left[z^{n}\right] H(x, 1, z)}{\left[z^{n}\right] H(1,1, z)} \underset{n \rightarrow \infty}{=}\left(\frac{\rho(1)}{\rho(x)}\right)^{n+s_{0} / \sigma}\left[1+O\left(n^{-p / \sigma}\right)\right] .
$$

This say that $\chi_{n}(x)$ closely resembles the $n$th power of a fixed function, or, in probabilistic terms, that $A_{n}$ is formally like the sum of $n$ independent identically distributed random variables. A Gaussian law is then expected in the asymptotic limit. Precisely the Quasi-Powers Theorem of Hwang [55, 58] guarantees the existence of this limit law (the proof is an analytic analogue of the classical proof of the central limit theorem, based on the continuity theorem for characteristic functions); in addition, the speed of convergence estimate expressed by (62) translates automatically into an upper bound on the speed of convergence to the Gaussian limit (this fact results from a suitable use of the classical Berry-Esseen inequalities [27, 80]).

Note that the coefficients in the asymptotic forms of the mean and standard deviation of $A_{n}$ are accessible from $\rho^{\prime}(1)$ and $\rho^{\prime \prime}(1)$, themselves easily computable from the expansion of $\rho(x)$ at 1 in (56). (For instance, $\rho^{\prime}(1)=-\gamma /(\beta+\gamma)$.) We then have:

Proposition 9 Consider a semi-sacrificial urn. The number $A_{n}$ of $\mathrm{x}$-balls at time $n$ satisfies for $x$ in any compact set of the real line

$$
\mathbb{P}\left(\frac{A_{n}-\mu n}{\xi \sqrt{n}} \leq x\right)=\frac{1}{\sqrt{2 \pi}} \int_{-\infty}^{x} e^{-t^{2} / 2} d t+O\left(n^{-e}\right), \quad e=\min \left(\frac{p}{\sigma}, \frac{1}{2}\right),
$$

where the centering and scaling constants are

$$
\mu:=\lim _{n \rightarrow \infty} \frac{\mathbb{E}\left(A_{n}\right)}{n}=\frac{\sigma \gamma}{\beta+\gamma}, \quad \xi^{2}:=\lim _{n \rightarrow \infty} \frac{\mathbb{V}\left(A_{n}\right)}{n}=\frac{\beta \gamma}{-\alpha+\beta+2 \gamma}\left(\frac{\alpha-\gamma}{\beta+\gamma}\right)^{2} \sigma .
$$

(x) The functions $S, C$ do have conjugate singularities at $\rho_{j}=\rho \omega^{j}$. However, none of these, except $\rho$ itself, plays a rôle. Indeed, the singularity $\rho_{j}$ of $S, C$ induces for $H(x, 1, z)$ a singularity at

$$
\rho_{j}(x)=\frac{\rho_{j}-J\left(x^{-\alpha} \Delta(x)^{\alpha}\right)}{-\alpha \Delta(x)^{\sigma}}
$$

Apart from $\rho_{0}(x) \equiv \rho(x)$, all the other $\rho_{j}(x)$ escape at infinity as $x \rightarrow 1$, and, in particular, do not contribute in the scale of the problem, once $x$ is restricted to a sufficiently small neighbourhood of 1 . 


\subsubsection{Moments}

Our purpose here is to establish that moments of any order $r \in \mathbb{Z}_{\geq 1}$ of the urn's composition $A_{n}$ are expressible in hypergeometric form in the following sense.

Definition 4 A function $t(n)$ defined for integer values of its argument is said to be a hypergeometric term if $t(n+1) / t(n)$ is a rational function of $n$. It is said to be of hypergeometric form or type if it is a $\mathbb{C}$-linear combination of a finite number of hypergeometric terms.

Bivariate generating functions are a convenient way to get access to moments. Set

$$
\chi_{r}(z):=\left.\frac{\partial^{r}}{\partial x^{r}} H(x, 1, z)\right|_{x=1}=r !\left[(x-1)^{r}\right] H(x, 1, z),
$$

where the last form indicates that $H(x, 1, z)$ should be expanded at $x=1$. The $r$ th factorial moment of $A_{n}$ is then

$$
\mathbb{E}\left(A_{n}\left(A_{n}-1\right) \cdots\left(A_{n}-r+1\right)\right)=\frac{\left[z^{n}\right] \chi_{r}(z)}{\left[z^{n}\right] \chi_{0}(z)} .
$$

The starting point of the analysid (xi) is an extended version of the expansion (60), namely

$$
H(x, 1, z)=(\sigma(\rho(x)-z))^{-s_{0} / \sigma} \mathcal{A}\left(\sigma(\rho(x)-z)^{p / \sigma}\right)
$$

where $\mathcal{A}(w)$ is an analytic function of $w$ at 0 . (Analyticity derives from the fact that $\mathcal{A}$ is obtained by composition and reversion of convergent Puiseux expansions; cf Lemma 2, Write $\mathcal{A}(w)=\sum_{k \geq 0} a_{k} w^{k}$ (one has $a_{0}=1$ and $a_{1}$ given by $(60)$ ). We have

$$
H(x, 1, z)=\sum_{k \geq 0} a_{k}\left(1-x^{p}\right)^{k}(\sigma(\rho(x)-z))^{\left(-s_{0}+k p\right) / \sigma} .
$$

The $r$ th moment is obtained by applying $\partial_{x}^{r}$ followed by the substitution $x \rightarrow 1$. Clearly, only the terms corresponding to $k=0, \ldots, r$ need to be considered.

Now, we have the reduction

$$
\left.(\sigma(\rho(x)-z))^{-\theta}\right|_{x=1}=(1-\sigma z)^{-\theta}
$$

as is seen from setting $x=1$ in (64), given the fact that $H(1,1, z)$ is the univariate GF of all urn histories given by (5). It then results that, for any $m \geq 0$, the quantity

$$
\partial_{x}^{m}\left(\left.\sigma(\rho(x)-z)^{-\theta}\right|_{x=1}\right.
$$

is a linear combination of terms of the form $(1-\sigma z)^{-\theta+\ell}$, for $\ell=0 \ldots m$. Here, the exponents $\theta$ to be considered are

$$
\theta \in\left\{\frac{-s_{0}+k p}{\sigma} \mid k=0 . . r\right\} .
$$

Thus, the quantity

$$
\left.\partial_{x}{ }^{r} H(x, 1, z)\right|_{x=1}=\sum_{j=0}^{r} c_{j, r}(1-\sigma z)^{-\theta+r},
$$

is the sum of a finite number of terms of the form $(1-\sigma z)^{-\xi}$. Coefficient extraction based on the rule

$$
\left[z^{n}\right](1-\sigma z)^{-\xi}=\sigma^{n}\left(\begin{array}{c}
n+\xi-1 \\
\xi-1
\end{array}\right)
$$

then shows the following:

Proposition 10 All moments of a semi-sacrificial urn are of hypergeometric form in the sense of Definition 4

(xi) This derivation follows closely the treatment of fully sacrificial urns in [30. p. 1216-1217]. 
Note 9. On moments. Another way to gain access to moments is via the partial differential equation satisfied by $H(x, 1, z)$. This has the advantage of applying to all balanced $2 \times 2$ urn models. Start from the partial differential equation (the PDE of (14), with $\mathfrak{D}$ the operator of (9)) $\partial_{z} H=\mathfrak{D} H$, where, momentarily, $H$ represents the trivariate GF of urn histories. Using homogeneity relations, we know that it is possible to eliminate the $\partial_{y} H$ term, and then set $y=1$. There results the equation (which repeats 16 )

$$
\left(x^{\alpha+1}-x^{\gamma+1}\right) \partial_{x} \bar{H}=-s_{0} x^{\gamma} \bar{H}+\left(1-\sigma x^{\gamma} z\right) \partial_{z} \bar{H},
$$

where $\bar{H}=H(x, 1, z)$. First, it is possible to set $x=1$ in 65 . We then retrieve the GF $H(1,1, z)$ via the solution of a homogeneous differential equation and find, as expected $H(1,1, z)=(1-\sigma z)^{-s_{0} / \sigma}$. Next, if we apply differentiation $\partial_{x}{ }^{r}$, we obtain a linear PDE for $\partial_{x}{ }^{r} H(x, 1, z)$, which, upon setting $x=1$ provides $\chi_{r}(z)$ as the solution of an inhomogeneous differential equation of order 1 . The homogeneous equation is of the same type as that satisfied by $H(1,1, z)$. Then, the variation-of-constant method is applicable. Thus the GF of any moment for any $2 \times 2$ balanced urn model (not only sacrificial ones!) is expressible by quadratures. This is an illustration of the "moment pumping" method (see [35]), where moments are pumped out of a functional equation for a BGF. We do not pursue this thread here since the method appears to be more involved than the plain perturbation techniques employed in the paper. (It would also be of interest to relate this approach to Pouyanne's methods [91] 90].)

\subsubsection{Large deviations}

The discussion of large deviations (see [19] for an accessible introduction) follows here the same lines as in the treatment of fully sacrificial urns by [30]. It is based on the general fact that knowledge of singularities of a bivariate generating functions as the secondary parameter $x$ varies does provide exponential tail bounds [38, 39, 56, 57] on the associated distributions.

The exact representation

$$
\mathbb{P}\left(A_{n} \leq k\right)=\left[x^{k}\right]\left(\frac{1}{1-x} \frac{\left[z^{n}\right] H(x, 1, z)}{\left[z^{n}\right] H(1,1, z)}\right),
$$

and the estimate

$$
\frac{\left[z^{n}\right] H(x, 1, z)}{\left[z^{n}\right] H(1,1, z)}=\frac{C(x)}{C(1)}\left(\frac{\rho(1)}{\rho(x)}\right)^{n}(1+o(1))
$$

imply the family of bounds valid for $x \in(0,1)$ :

$$
\mathbb{P}\left(A_{n} \leq k\right) \leq \frac{1}{x^{k}(1-x)} \frac{C(x)}{C(1)}\left(\frac{\rho(1)}{\rho(x)}\right)^{n}(1+o(1)) .
$$

(Use the fact that, for $\phi$ an analytic function with nonnegative coefficients, one has $\left[x^{k}\right] \phi(x) \leq \phi(x) x^{-k}$ when $x>0$ lies within the disc of convergence of $\phi$.) The large deviation case (for left tails, when $k=\kappa n$ is less than the mean $\mathbb{E}\left(A_{n}\right) \sim \mu n$, that is, $\left.\kappa<n\right)$, is quantified by choosing the particular value $x_{0}$ of $x$ that optimizes the family of bounds 66 . This motivates the following statement.

Proposition 11 For a semi-sacrificial urn and for a number $\kappa<\mu$ one has the large deviation estimate

$$
\lim _{n \rightarrow \infty} \frac{1}{n} \log \mathbb{P}\left(A_{n} \leq \kappa n\right)=\log \left(\frac{\rho(1)}{x_{0}^{\kappa} \rho\left(x_{0}\right)}\right)
$$

where $x_{0}$ is the root in $(0,1)$ of the equation

$$
-\frac{x_{0} \rho^{\prime}\left(x_{0}\right)}{\rho\left(x_{0}\right)}=\kappa
$$

A similar estimate holds for the right tails, $\mathbb{P}\left(A_{n} \geq \kappa n\right)$ with $\kappa>\mu$.

Proof (sketch): The upper bound is provided by the preceding discussion. That the upper bound is tight follows from an argument of Cramér-type, which has been placed within the general framework of QuasiPowers approximations by Hwang [56, 57]. (The essential idea is to develop a central limit law, again based on Quasi-Powers, for an exponentially shifted family of distributions that includes the distribution of $A_{n}$.) 


\subsection{The algebraic family $[-1, \sigma+1, \sigma-1,1]$}

We now conduct a search for semi-sacrificial urns whose generating function is elementary. In particular, our goal is to find classes of urns such that the $S$ and $C$ functions reduce to classical functions of analysis. A sufficient condition is that the integral in (35), on which the $J$ function of Definition 3 is based, be an algebraic function.

In accordance with (35), we consider the integral

$$
I(u):=\int_{c}^{u} \frac{w^{-\alpha-1}}{\left(1+w^{p}\right)^{\beta / p}} d w .
$$

(The choice of $c$ is immaterial here.) For a semi-sacrificial urn, we have $\alpha<0$. An immediate sufficient condition for explicit integrability is that $w^{-\alpha-1} d w$ be (up to a constant factor) the differential element corresponding to the quantity $w^{p}$ in the denominator. Such is the case if $p=-\alpha$, that is

$$
\gamma-\alpha=-\alpha
$$

implying $\gamma=0$. This means that the matrix $\mathcal{M}$ is triangular-this case, which is incompatible with the conditions (47), requires a special treatment that is given in 87

An alternative condition for explicit integrability is obtained by reduction to the previous case, after the change of variables $v=1 / w$. Proceeding in this way, we find

$$
I(u)=\int_{1 / c}^{1 / u} \frac{v^{\alpha-1}}{\left(1+v^{-p}\right)^{\beta / p}} d v=\int_{1 / c}^{1 / u} \frac{v^{\alpha+\beta-1}}{\left(1+v^{p}\right)^{\beta / p}} d v .
$$

Thus, explicit integrability is granted if $\sigma=\alpha+\beta$ and $p=\gamma-\alpha$ are equal. Algebraically, this corresponds to the two parameter family of matrices $\left(\begin{array}{cc}\alpha & \gamma-2 \alpha \\ \gamma & -\alpha\end{array}\right)$. Now, since we have $\alpha<0$, the tenability conditions require that $|\alpha|$ should divide $\gamma$, say $\gamma=-d \alpha$. We only consider arithmetically irreducible matrices, so that we only need to retain the case $\alpha=-1$. The matrices of interest are thus of the form

$$
\left(\begin{array}{cc}
-1 & \sigma+1 \\
\sigma-1 & 1
\end{array}\right)
$$

and we know a priori that $I$, hence $J$, hence $S$ and $C$, are each a simple algebraic function. It is pleasant to see that the general class of matrices (67) encapsulate the urn of Example 3 (p. 78), which is obtained by setting $\sigma=2$.

Example 5. The second algebraic urn $[-1,4,2,1]$. We have corresponding to $\sigma=3$

$$
\mathcal{M}=\left(\begin{array}{cc}
-1 & 4 \\
2 & 1
\end{array}\right), \quad p=3, \lambda \equiv \frac{\beta}{p}=\frac{4}{3} .
$$

The fundamental integral is

$$
J(u) \equiv \int_{0}^{u} \frac{d \zeta}{\left(1+\zeta^{3}\right)^{4 / 3}}=\frac{u}{\left(1+u^{3}\right)^{1 / 3}} .
$$

The function $S(z)$ is the solution of $J(S(z))=z$, that is, $S /\left(1+S^{3}\right)^{1 / 3}=z$, which is readily solved to provide $S$ and then $C$ :

$$
S(z)=\frac{z}{\left(1-z^{3}\right)^{1 / 3}}, \quad C(z)=\left(1-z^{3}\right)^{1 / 3} .
$$

(Notice the striking parallel with the corresponding function in Example 3) The GF of urn histories is then found to be

$$
H(x, 1, z)=\frac{\left(1-x^{3}\right)^{s_{0} / 3}\left(x+z\left(1-x^{3}\right)\right)^{a_{0}}}{\left(1-\left(x+z\left(1-x^{3}\right)\right)^{3}\right)^{s_{0} / 3}} .
$$

The radius of convergence $\rho(x)$, which determines the main characteristics of the distribution of $A_{n}$ is then found to be

$$
\rho(x)=\frac{1}{1+x+x^{2}},
$$

which, as predicted by Theorem 3 is an analytic function of $x$ for $x$ near 1 . In this case, the large deviation rate function can be made fully explicit in terms of simple radicals.

The computations in the last example are typical. We state:

Proposition 12 Any urn having a matrix of the form (67) with $\sigma \geq 2$ is such that the bivariate generating function of histories is an algebraic function expressible by radicals:

$$
H(x, 1, z)=\frac{\left(1-x^{\sigma}\right)^{s_{0} / \sigma}\left(x+z\left(1-x^{\sigma}\right)\right)^{a_{0}}}{\left(1-\left(x+z\left(1-x^{\sigma}\right)\right)^{\sigma}\right)^{s_{0} / \sigma}} .
$$




\subsection{The algebraic family $[-1, \sigma+1,1, \sigma-1]$ ( $\sigma$ even)}

When aiming at integrability, a good thing to do is look for small values of the parameter $r$ since the integrand of the fundamental integral $J(u)$ is $\left(1+\zeta^{r}\right)^{-\lambda}$. For semi-sacrificial urns, the case $r=1$ is irrelevant, so that we try next $r=2$.

Example 6. The third algebraic urn $[-1,5,1,3]$. The parameters are $r=2$ and $\lambda=5 / 2$, the balance being $\sigma=4$. The fundamental integral

$$
J(u)=\int_{0}^{u} \frac{d \zeta}{\left(1+\zeta^{2}\right)^{5 / 2}}=\frac{u\left(3+2 u^{2}\right)}{3\left(1+u^{2}\right)^{3 / 2}}
$$

is then an algebraic function. Accordingly, the function $S(z)$, which solves $J(S(z))=z$, satisfies

$$
\frac{S^{2}\left(3+2 S^{2}\right)^{2}}{3\left(1+S^{2}\right)^{3}}=z^{2}
$$

which means that its square $S^{2}$ is a cubic algebraic function in $z^{2}$. Its expansion could then be made explicit by means of Lagrange inversion.

The previous example suggests considering the class

$$
\left(\begin{array}{cc}
-1 & \sigma+1 \\
1 & \sigma-1
\end{array}\right)
$$

and, specifically, restrict attention to the case when $\sigma$ is even. Note that the case $\sigma=2$ also includes the first algebraic urn. (The case $\sigma=2$ resorts to both of the schemes 67) and 68.) For $\sigma=2 \tau$, it is seen (using integration by parts) that $J(u)$ is of the form

$$
J(u)=\frac{u P_{\tau}\left(u^{2}\right)}{\left(1+u^{2}\right)^{\tau-1 / 2}},
$$

where $P_{\tau}$ is a polynomial of degree $\tau-1$. As a consequence:

Proposition 13 Any urn having a matrix of the form (68) with $\sigma \geq 2$ is such that the bivariate generating function of histories is an algebraic function. In particular, the pseudo-sine function is such that its square is the inverse of a rational function.

\section{The class of $2 \times 2$ fully sacrificial urns $(\alpha, \delta<-1)$}

Fully sacrificial urns are such that both diagonal entries in the matrix are negative:

$$
\mathcal{M}=\left(\begin{array}{cc}
\alpha & \beta \\
\gamma & \delta
\end{array}\right), \quad \alpha<0, \quad \delta<0, \quad \beta \gamma \neq 0, \quad \sigma>0 .
$$

These models are the main object of study of the article by Flajolet, Gabarró and Pekari [30], of which the present works constitutes in many ways a follow-up. We give below a brief account of this paper under our current perspective.

\subsection{Analytic solutions}

The first integral of Proposition 7 (see $\$ 4$ p. 74) applies to the fully sacrificial case, so that the formal treatment of semi-sacrificial urns (\$5) can be adapted easily to the fully sacrificial case. In fact, the formal analysis of [30] was based on the partial differential equation of the complete GF of urn histories as discussed in Note 1 (see p. 66 and Equation (14)). That partial differential equation was then solved by the method of characteristics, an hitherto neglected tool in the analysis of urn models. In our view, the present treatment, via the Isomorphism Theorem (Theorem 1) allows for a more transparent connection between the urn's structure and its analytic solution, since it bypasses a somewhat opaque computational procedure.

The next phase of [30] consists in working out properties of the $S, C$ functions as regards their singularities. (The corresponding functions are called there $\psi_{I}$ and $\psi_{I I}$.) The fact that both $\alpha$ and $\delta$ are now negative entails that the conformal mapping properties of the base functions are comparatively simple to elucidate: contrary to the situation encountered with semi-sacrificial urns in $\$ 5$ there are no occurrences of multiple coverings, so that suitable sectors of the complex $z$-plane are found to be mapped to well-determined quadrilaterals in the image complex plane (these are the "elementary kites" of [30]). In such a case, dominant singularities can be found directly, without resorting to the indirect argument employed in the proof of Lemma 1, p. 81 .

The analytic machinery needed to grind probabilistic properties from singularities is then identical to the one exposed in the previous section, $₫ 5$. One obtains in this way a result that closely parallels Theorem 3 . 


\begin{tabular}{|c|c|c|c|c|}
\hline & $(\mathcal{M})$ & $(\Sigma)$ & (D) & Type \\
\hline $\mathcal{A}$ & $\left(\begin{array}{ll}-2 & 3 \\
4 & -3\end{array}\right)$ & $\dot{x}=x^{-1} y^{3}, \dot{y}=x^{4} y^{-2}$ & $x^{-1} y^{3} \partial_{x}+x^{4} y^{-2} \partial_{y}$ & $\wp[30,88$ \\
\hline $\mathcal{B}$ & $\left(\begin{array}{ll}-1 & 2 \\
3 & -2\end{array}\right)$ & $\dot{x}=y^{2}, \dot{y}=x^{3} y^{-1}$ & $y^{2} \partial_{x}+x^{3} y^{-1} \partial_{y}$ & Jacobian 30 \\
\hline $\mathcal{C}$ & $\left(\begin{array}{ll}-1 & 2 \\
2 & -1\end{array}\right)$ & $\dot{x}=y^{2}, \dot{y}=x^{2}$ & $y^{2} \partial_{x}+x^{2} \partial_{y}$ & Dixonian [16 \\
\hline $\mathcal{D}$ & $\left(\begin{array}{ll}-1 & 3 \\
3 & -1\end{array}\right)$ & $\dot{x}=y^{3}, \dot{y}=x^{3}$ & $y^{3} \partial_{x}+x^{3} \partial_{y}$ & lemniscatic 30 \\
\hline $\mathcal{E}$ & $\left(\begin{array}{ll}-1 & 3 \\
5 & -3\end{array}\right)$ & $\dot{x}=y^{3}, \dot{y}=x^{5} y^{-2}$ & $y^{3} \partial_{x}+x^{5} y^{-2} \partial_{y}$ & $\sqrt{\wp}$ \\
\hline $\mathcal{F}$ & $\left(\begin{array}{ll}-1 & 4 \\
5 & -2\end{array}\right)$ & $\dot{x}=y^{4}, \dot{y}=x^{5} y^{-1}$ & $y^{4} \partial_{x}+x^{5} y^{-1} \partial_{y}$ & \\
\hline
\end{tabular}

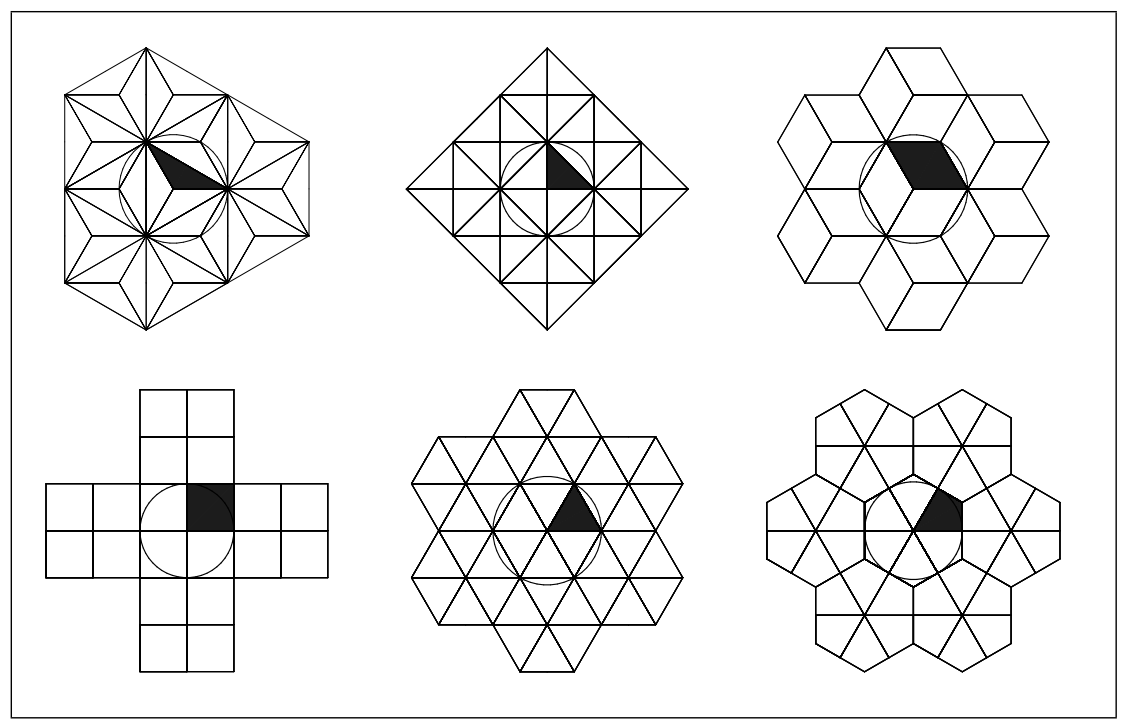

Figure 7: Top: The six elliptic $2 \times 2$ urns: matrix $(\mathcal{M})$, system $(\Sigma)$, partial differential operator $(\mathfrak{D})$, and elliptic type. Bottom: The corresponding six lattices $\left(\begin{array}{ccc}\mathcal{A} & B & C \\ \mathcal{D} & E & F\end{array}\right)$.

Theorem 4 (Fully sacrificial urns) Consider a fully sacrificial urn determined by a matrix satisfying the conditions of (69).

(i) The probability that, at time $n$, all balls are of the second type $\left(A_{n}=0\right)$ is exponentially small; this exponential rate is a quotient of Gamma function values taken at rational arguments.

(ii) The number of balls of the first type $\left(A_{n}\right)$ converges in distribution, in the large n limit, to a Gaussian law. The speed of convergence to the Gaussian limit is a negative fractional power of $n$.

(iii) The number $A_{n}$ of balls of the first type satisfies the mean and variance estimates: $\mathbb{E}\left(A_{n}\right) \sim \mu n$ and $\mathbb{V}\left(A_{n}\right) \sim \xi^{2} n$; in particular, $A_{n} / n$ converges in probability to $\mu$. Generally, any rth moment admits a closed-form expression, which is of hypergeometric type.

(iv) The number $A_{n}$ of balls of the first type satisfies a large deviation principle, with the large deviation rate function being effectively computable from the fundamental integral $J$ of the urn model.

Detailed statements that are analogous to Propositions 8 111 above are found in [30].

\subsection{Elliptic urns}

An elliptic function is a function that is meromorphic in the whole complex plane and is doubly periodic. There are several ways to develop the theory of such functions-accessible introductions appear in the books of Whittaker and Watson [103] and Chandrasekharan [13]. Elliptic functions also enjoy special conformal mapping properties.

One of the results of [30] amounts to characterizing all $2 \times 2$ urn models whose solutions can be expressed in terms of elliptic functions. It is found that there are exactly six such models (after arithmetical irreducibility, in the sense of [30, p. 74, is imposed), which are listed in Figure 7] [top]. Such elliptic urns are associated to the regular tilings of the Euclidean plane displayed in Figure 7 [bottom].

For our purposes, the most fruitful relations that determine elliptic functions are the differential ones. For the Weierstraß $\wp$-function, this is

$$
\wp^{\prime}(z)^{2}=4 \wp(z)^{3}-g_{2} \wp(z)-g_{3}, \quad \text { implying } \quad \wp^{\prime \prime}(z)=6 \wp(z)^{2}-\frac{1}{2} g_{2} .
$$



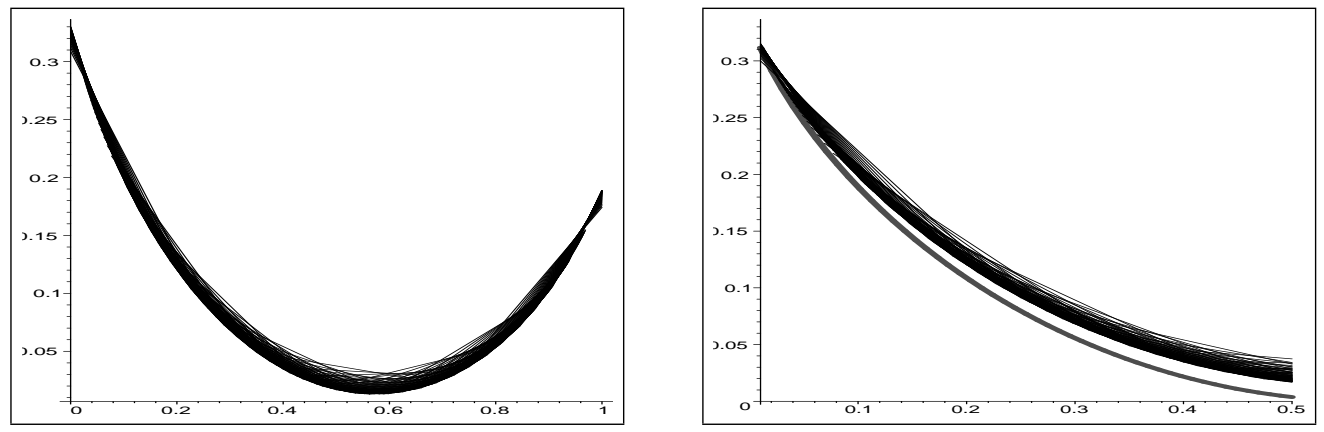

Figure 8: The 2-3 tree urn model. Left: a plot of $\left\{-\frac{1}{n} \log \mathbb{P}\left(A_{n}=k\right)\right\}_{k=0}^{n+1}$ against $k /(n+1)$, for $n=24 \ldots 96$; right: a comparison against the limit large deviation rate function.

see [103, Ch.XX]. The Jacobian elliptic function $\operatorname{sn}(z, k)$ satisfies the nonlinear differential equation

$$
\left(\frac{d y}{d z}\right)^{2}=\left(1-y^{2}\right)\left(1-k^{2} y^{2}\right)
$$

see [103, Ch.XXII]. Finally, an interesting differential system considered by Dixon [20], namely

$$
\left\{\begin{array}{l}
\dot{x}=y^{2} \\
\dot{y}=x^{2}
\end{array}, \quad\left\{\begin{array}{l}
x(0)=1 \\
y(0)=0
\end{array},\right.\right.
$$

has solutions, written $X(z)=\operatorname{cmh}(z)$ and $Y(z)=\operatorname{smh}(z)$ in the notations of [16], that parameterize the Fermat hyperbola $X^{3}-Y^{3}=1$ (this curve is of genus 1 , hence elliptic). We now briefly review some of the elliptic models of Figure 7, under the angle of differential relations and the isomorphism theorem.

A. The urn

$$
\mathcal{A}=\left(\begin{array}{cc}
-2 & 3 \\
4 & -3
\end{array}\right)
$$

(matrix $\mathcal{A}$ of Figure 77) is of great historical interest: it appears in the context of balanced 2-3 trees, a data structure of computer science used for sorting and searching [71]. The fringe analysis leads to this urn, the "2-3 tree urn"; see [2, 5, 106] as well as [1] for a "process viewpoint" and [83] for relations to rotation balanced data structures. Panholzer and Prodinger first derived the elliptic solution of this particular model [88], which then served as a prime motivation for the development of [30]. Explicit reductions to elliptic functions are developed in [30, 88], one of which goes as follows: strat from the associated differential system,

$$
\left\{\begin{array}{l}
\dot{x}=x^{-1} y^{3} \\
\dot{y}=x^{4} y^{-2}
\end{array}\right.
$$

then notice that $\dot{x} x=y^{3}$, so that $x^{2}=2 \int y^{3}$; similarly, we have $y^{3}=3 \int x^{4}$ which yields $x^{2}=\iint x^{4}$. Setting $x^{2}=\Xi$ then shows that $\Xi^{\prime \prime}=6 \Xi^{2}$, which is an avatar of the differential equation (70) satisfied by the Weierstraß $\wp$ function.

Figure 8 displays numeric aspects of the large-deviation regime of such urns.

B. The urn

$$
\mathcal{B}=\left(\begin{array}{cc}
-1 & 2 \\
3 & -2
\end{array}\right)
$$

has an associated differential system

$$
\left\{\begin{array}{l}
\dot{x}=y^{2} \\
\dot{y}=x^{3} y^{-1}
\end{array},\right.
$$

which can be directly reduced to elliptic form like in the previous example. The second equation put under the form $y \dot{y}=x^{3}$ implies that $y^{2}=2 \int x^{3}$. Then, the first equation becomes $\dot{x}=\int x^{3}$, which by differentiation gives $\dot{x}=2 x^{3}$. This is then integrated upon multiplication by $\dot{x}$ : we have $\dot{x} \ddot{x}=2 x^{3} \dot{x}$ and $\frac{1}{2} \dot{x}^{2}=\frac{1}{2} x^{4}+\frac{1}{2} K$ for some constant $K$. The latter equation is of the Jacobian form (71).

C. The system (72) defining Dixonian functions is directly associated to the urn with matrix

$$
\mathcal{C}=\left(\begin{array}{cc}
-1 & 2 \\
2 & -1
\end{array}\right)
$$


(matrix $\mathcal{C}$ of Figure 77) discussed as Example 4. p. 79 under the name of "generation-parity model", with the functions smh, cmh providing the pseudo-sine $(S)$ and pseudo-cosine $(C)$ of the general theory. The combinatorics of these functions, including the reduction to the Weierstraß $\wp$ form and further properties of the urn model, are examined in [16].

D. The case of matrix $\mathcal{D}$ is reduced to elliptic form in [30]. What appears in this case is the lemniscatic integral

$$
I(u)=\int_{0}^{u} \frac{d \zeta}{\sqrt{1-\zeta^{4}}} .
$$

E. For the urn and associated system

$$
\mathcal{E}=\left(\begin{array}{cc}
-1 & 3 \\
5 & -3
\end{array}\right), \quad\left\{\begin{array}{l}
\dot{x}=y^{3} \\
\dot{y}=x^{5} y^{-2}
\end{array}\right.
$$

one can start the reduction like in Case $\mathbf{C}$ above. One finds $y^{3}=3 \int x^{5}$, so that $\dot{x}=3 \int x^{5}$, implying $\ddot{x}=3 x^{5}$ and $\dot{x}^{2}=x^{6}+K$. This is not immediately recognizable as being of elliptic type. However, set $\Xi=x^{2}$. We find $\dot{\Xi}^{2}=4 \Xi^{3}+4 K$, which is now seen to be of elliptic type. Thus, $\Xi(t)$ is an elliptic function and $x$ is the square-root of an elliptic function. (It is still justified to name the urn "elliptic", since the initial conditions $a_{0}=2, b_{0}=0$, for instance, lead to bona fide elliptic function solutions.)

F. The reduction of the matrix $\mathcal{F}$ to elliptic form is discussed briefly in [30].

Let us finally mention that a $3 \times 3$ model, which is exactly solvable in terms of Jacobian functions, is discussed in 9.4 . p. 107 (the pelican's urn).

\section{Triangular $2 \times 2$ urns $(\gamma=0)$}

In this section, we examine balanced urn models whose replacement rules are determined by a triangular matrix (xii),

$$
\mathcal{M}=\left(\begin{array}{cc}
\alpha & \sigma-\alpha \\
0 & \sigma
\end{array}\right), \quad 0<\alpha<\sigma .
$$

(Thus, with our general notations: $\gamma=0$, and $\delta=\sigma$ is the balance of the urn.)

Triangular urns may be viewed as a simple model for evolving species. Consider two populations, for instance apes and human. At time $n$, a randomly chosen individual gives birth to $\sigma$ children. Apes may evolve and as a consequence give birth to children of both species. But since offsprings inherit the characteristics of their parents, a human can only give birth to a human (this is perhaps an optimistic model of reality). The evolution of both populations can then be represented by such a triangular urn model.

We establish here the following properties, whose detailed statements constitute Propositions 14

Theorem 5 (Triangular $2 \times 2$ urns) Consider a triangular urn model determined by a matrix of the form (73).

(i) The complete generating function of urn histories is an algebraic function of a special radical form. A single combinatorial sum expresses exactly the composition of the urn at any instant.

(ii) Moments of any order are expressible as finite linear combinations of products and quotients of binomial coefficients (i.e., they are of hypergeometric type).

(iii) Both a central and a local limit hold for the urn's composition, the distribution being expressible in terms of the stable laws of probability theory.

(iv) Moments of fractional orders and the Mellin transform of the limit law are expressible as quotients of Gamma function values.

Bagchi and Pal [6, p. 395] briefly mention the triangular urn model, which, in their terms, "requires further investigation" and "presents some curious technical problems and appears to need a separate treatment". The model was later studied by Gouet [47] who established convergence in distribution but did not characterize the limit, due to limitations arising from the non-constructive character of martingale theory. Kotz, Mahmoud and Robert [74] found the exact (hypergeometric) form of the expectation of the number of balls of the first color at time $n$ for the particular urn $\left(\begin{array}{ll}1 & 3 \\ 0 & 4\end{array}\right)$. An original analysis of $2 \times 2$ triangular urns along the lines of the present treatment was included in the first version (dated March 2003) of [30], but it was restricted to urns of the form $\left(\begin{array}{cc}\sigma-1 & 1 \\ 0 & \sigma\end{array}\right)$, and for reasons of size, had to be kept out of the eventually published version [30]. Puyhaubert and Flajolet then worked out the case of a general matrix of the form [73,

\footnotetext{
(xii) The case $\alpha=\sigma$ leads back to the original urn studied by Pólya; the case $\alpha=0$ is such that the urn's composition is deterministic: see the "records' urn" in 2.6 the case $\alpha<0$ is such that balls of type $x$ disappear almost surely, so that the evolution is eventually trivial. Higher dimensional urns described by a triangular matrix are also of interest since they form an integrable family.
} 
with the corresponding results being reported in the thesis [92]. In the mean time, Janson [63] developed a wide-encompassing theory of triangular urn models, including non-balanced ones. To this class of triangular models, we contribute explicit formulae, speed of convergence estimates, as well as a local limit law. Note finally, that Gnedin and Pitman have recently encountered similar generating functions in [45].

\subsection{Generating functions and the exact form of probabilities}

Our starting point is the triangular matrix (73) and the main tool is the isomorphism theorem (Theorem 1 . p. 65. We assume $a_{0}>0$, since otherwise the urn would never contain a ball of the first type. Regarding exact expressions of probabilities, we have:

Proposition 14 Consider a triangular urn of the form (73), whose composition at time 0 is $\left\langle a_{0}, b_{0}\right\rangle$. The bivariate generating function of urn histories is an algebraic function expressible by radicals:

$$
H(x, 1, z)=x^{a_{0}}(1-\sigma z)^{-b_{0} / \sigma}\left(1-x^{\alpha}\left(1-(1-\sigma z)^{\alpha / \sigma}\right)\right)^{-a_{0} / \alpha}
$$

The probability that the urn contains $a_{0}+k \alpha$ black balls at time $n$ is

$$
\mathbb{P}\left[A_{n}=a_{0}+k \alpha\right]=\frac{\Gamma(n+1) \Gamma\left(\frac{s_{0}}{\sigma}\right)}{\Gamma\left(\frac{s_{0}}{\sigma}+n\right)}\left(\begin{array}{c}
k+\frac{a_{0}}{\alpha}-1 \\
k
\end{array}\right) \sum_{i=0}^{k}(-1)^{i}\left(\begin{array}{l}
k \\
i
\end{array}\right)\left(\begin{array}{c}
n+\frac{b_{0}-\alpha i}{\sigma}-1 \\
n
\end{array}\right) .
$$

Proof: Triangular urns are most conveniently treated by means of the associated differential system that reads:

$$
\left\{\begin{array}{l}
\dot{x}=x^{\alpha+1} y^{\sigma-\alpha} \\
\dot{y}=y^{\sigma+1}
\end{array}, \quad\left\{\begin{array}{l}
x(0)=x_{0} \\
y(0)=y_{0}
\end{array} .\right.\right.
$$

The system is triangular, so that one can first solve for $y$, resulting in

$$
Y(t)=y_{0}\left(1-\sigma y_{0}^{-\sigma} t\right)^{-1 / \sigma}
$$

Then $X(t)$ must satisfy:

$$
\dot{x} x^{-\alpha-1}=y^{\sigma-\alpha},
$$

which is explicitly integrable. Hence, the solution to the system is explicit and it provides the algebraic function of 74,.

By definition, only blocks of $\alpha$ balls can be added to the urn, so that $A_{n}$ is necessarily of the form $A_{n}=a_{0}+k \alpha$ for some $k \in \mathbb{Z}_{\geq 0}$. Coefficient extraction from 74 based on the expansion of $(1-X)^{-\alpha_{0} / a}$ then yields

$$
\left[x^{a_{0}+k \alpha}\right] H(x, 1, z)=\left(\begin{array}{c}
k+\frac{a_{0}}{\alpha}-1 \\
k
\end{array}\right)(1-\sigma z)^{-b_{0} / \sigma}\left(1-(1-\sigma z)^{\alpha / \sigma}\right)^{k} .
$$

The formula (75) then follows after expanding the $k$ th power, upon performing coefficient extraction with respect to $z$.

Example 7. The simplest triangular urn: $[1,1 ; 0,2]$. The triangular matrix

$$
\mathcal{M}=\left(\begin{array}{ll}
1 & 1 \\
0 & 2
\end{array}\right)
$$

with $\sigma=2$, gives rise to the simplest nontrivial triangular model. The BGF of urn histories is

$$
H(x, 1, z)=x^{a_{0}}(1-2 z)^{-b_{0} / 2}(1-x(1-\sqrt{1-2 z}))^{-a_{0}}
$$

which leads to

$$
\left[x^{k}\right] H(x, 1, z)=\left(\begin{array}{c}
k-1 \\
a_{0}-1
\end{array}\right)(1-2 z)^{-b_{0} / 2}(1-\sqrt{1-2 z})^{k-a_{0}} .
$$

Consider the case $b_{0}=0$ and (for convenience) $a_{0}=1$. Now, the function $\phi(z)=1-\sqrt{1-2 z}$ is such that its powers can be explicitly expanded by means of the Lagrange Inversion Theorem, since it satisfies the implicit definition $\phi=z /(2-\phi)$. We thus find $(n \geq 1$ and $2 \leq k \leq n+1)$

$$
\mathbb{P}\left[A_{n}=k\right]=\frac{k-1}{n} 2^{k-1} \frac{\left(\begin{array}{c}
2 n-k \\
n-1
\end{array}\right)}{\left(\begin{array}{c}
2 n \\
n
\end{array}\right)},
$$


under the initial condition $\left(a_{0}, b_{0}\right)=(1,0)$. Asymptotically, the distribution is of the Rayley type,

$$
\mathbb{P}\left(a_{n}=\lfloor\xi \sqrt{n}\rfloor\right) \sim \frac{\xi}{2 \sqrt{n}} e^{-\xi^{2} / 4},
$$

uniformly for $\xi$ in any compact set of $\mathbb{R}_{>0}$, this fact also constitutes a special case of Proposition 16 below. This urn was studied independently by Janson who obtains a central limit result [63, Ex. 3.1].

\subsection{Moments}

Like probabilities, moments of the number of x-balls admit closed-form expressions: these are even simpler, as each moment turns out to be of hypergeometric type, that is, a finite linear combination of products and quotients of Gamma function values (see Definition 4, p. 87). For an alternative approach, see [90, §3.2].

Proposition 15 The random variable $A_{n}$ has expectation and standard deviation that satisfy

$$
\begin{aligned}
& \mathbb{E}\left[A_{n}\right]=a_{0} \frac{\Gamma\left(\frac{t_{0}}{s}\right)}{\Gamma\left(\frac{t_{0}+\alpha}{s}\right)} n^{\alpha / s}+O\left(n^{\alpha / \sigma-1}\right) \\
& \left(\sigma\left[A_{n}\right]\right)^{2}=a_{0}\left[\left(a_{0}+\alpha\right) \frac{\Gamma\left(\frac{t_{0}}{s}\right)}{\Gamma\left(\frac{t_{0}+2 \alpha}{s}\right)}-a_{0}\left(\frac{\Gamma\left(\frac{t_{0}}{s}\right)}{\Gamma\left(\frac{t_{0}+\alpha}{s}\right)}\right)^{2}\right] n^{2 \alpha / s}+O\left(n^{\alpha / \sigma}\right) .
\end{aligned}
$$

The general moment of order $\ell$ is a finite linear combination of terms of the form

$$
\frac{\left(\frac{t_{0}+k \alpha}{s}\right) \cdots\left(\frac{t_{0}+k \alpha}{s}+n-1\right)}{\frac{t_{0}}{s} \cdots\left(\frac{t_{0}}{s}+n-1\right)}=\frac{\left(\frac{t_{0}+k \alpha}{s}+n-1\right)}{\left(\begin{array}{c}
\frac{t_{0}}{s}+n-1 \\
n
\end{array}\right)}, \quad 0 \leq k \leq \ell,
$$

and asymptotically,

$$
E\left[A_{n}^{\ell}\right]=\alpha^{\ell} \frac{\Gamma\left(\frac{a_{0}+\ell \alpha}{\alpha}\right) \Gamma\left(\frac{t_{0}}{s}\right)}{\Gamma\left(\frac{a_{0}}{\alpha}\right) \Gamma\left(\frac{t_{0}+\ell \alpha}{s}\right)} n^{\ell \alpha / s}+O\left(n^{(\ell-1) \alpha / \sigma}\right)
$$

Proof: Given the probability generating function of $A_{n}$ (Proposition[14), moments are obtained by repeated differentiation with respect to variable $u$ followed by the assignment $u \mapsto 1$. Precisely, the $\ell$ th factorial moment is equal to

$$
E\left[A_{n}\left(A_{n}-1\right) \cdots\left(A_{n}-\ell+1\right)\right]=\frac{\Gamma(n+1) \Gamma\left(\frac{t_{0}}{\sigma}\right)}{\sigma^{n} \Gamma\left(\frac{t_{0}}{\sigma}+n\right)}\left[z^{n}\right]\left[\frac{\partial^{\ell}}{\partial u^{\ell}} H(x, 1, z)\right]_{u=1} .
$$

For the first moment, this gives us

$$
E\left[A_{n}\right]=\frac{\Gamma(n+1) \Gamma\left(\frac{t_{0}}{\sigma}\right)}{\sigma^{n} \Gamma\left(\frac{t_{0}}{\sigma}+n\right)}\left[z^{n}\right]\left(\frac{\partial H(x, 1, z)}{\partial u}\right)_{x=1}=\frac{\Gamma(n+1) \Gamma\left(\frac{t_{0}}{\sigma}\right)}{\sigma^{n} \Gamma\left(\frac{t_{0}}{\sigma}+n\right)}\left[z^{n}\right] a_{0} \Delta^{t_{0}+a}
$$

where $\Delta$ denotes here the function $\Delta=(1-\sigma z)^{-1 / \sigma}$. For the second moment, one finds

$$
E\left[A_{n}^{2}\right]=\frac{\Gamma(n+1) \Gamma\left(\frac{t_{0}}{\sigma}\right)}{\sigma^{n} \Gamma\left(\frac{t_{0}}{\sigma}+n\right)}\left[z^{n}\right]\left(a_{0}\left(a_{0}+a\right) \Delta^{t_{0}+2 a}-a_{0} a \Delta^{t_{0}+a}\right) .
$$

Coefficient extraction leads to binomial coefficient forms involving rational parameters. The asymptotic equivalents are obtained by Stirling's formula, which proves (76).

Similarly, for higher moments, we start by expressing the function $H(x, 1, z)$ as

$$
H(x, 1, z)=\Delta^{t_{0}}\left(1-\Delta^{a}\left(1-x^{-a}\right)\right)^{-a_{0} / a} .
$$

For $x$ close enough to 1 , this expression admits a series expansion given by

$$
H(x, 1, z)=\Delta^{t_{0}} \sum_{k=0}^{\infty} \frac{a_{0}}{a} \cdots\left(\frac{a_{0}}{a}+k-1\right) \Delta^{k a} \frac{\left(1-x^{-a}\right)^{k}}{k !} .
$$


Introduce the collection of numbers $\left(s_{k, \ell}\right)_{k, \ell \in \mathbb{Z}_{\geq 0}}$ such that for $x$ near 1 ,

$$
\left(1-x^{-\alpha}\right)^{k}=\sum_{\ell=k}^{\infty} s_{k, \ell}(x-1)^{\ell} .
$$

Then, $s_{k, k}=\alpha^{k}$ and upon exchanging summations, one finds

$$
H(x, 1, z)=\Delta^{t_{0}} \sum_{\ell=0}^{\infty}(x-1)^{\ell}\left(\sum_{k=0}^{\ell} \frac{s_{k, \ell}}{k !} \frac{a_{0}}{\alpha} \cdots\left(\frac{a_{0}}{\alpha}+k-1\right) \Delta^{k \alpha}\right) .
$$

From this expression, it is seen that the $\ell$ th derivative of $H(x, 1, z)$ at $x=1$ is a polynomial in $\Delta$, of degree $t_{0}+\ell \alpha$, whose leading coefficient is $a_{0} \cdots\left(a_{0}+(k-1) \alpha\right)=\alpha^{\ell} \Gamma\left(\frac{a_{0}}{\alpha}+\ell\right) / \Gamma\left(\frac{a_{0}}{\alpha}\right)$. By linearity, this property also extends to the $\ell$ th power moment. Finally, since

$$
\left[z^{n}\right](1-z)^{-\lambda_{1}}=o\left(\left[z^{n}\right](1-z)^{-\lambda_{2}}\right) \quad \text { if } \quad \lambda_{1}<\lambda_{2}
$$

the asymptotic equivalent of the general moment follows.

Example 8. The KMR triangular urn $[1,3 ; 0,4]$. In [74], Kotz, Mahmoud, and Robert discuss recurrences satisfied by probabilities and moments in the triangular case and make explicit some first moment computations. In particular, they study a variant of the model

$$
\mathcal{M}=\left(\begin{array}{ll}
1 & 3 \\
0 & 4
\end{array}\right)
$$

and we name the latter the KMR triangular urn. We consider here the initial conditions $\left(a_{0}, b_{0}\right)=(1,0)$. The mean number of balls of the x-type at time $n$ satisfies

$$
\begin{aligned}
\mathbb{E}\left[A_{n}\right] & =4 n+1-\frac{1}{\left(\begin{array}{c}
n-3 / 4 \\
n
\end{array}\right)} \\
& \sim 4 n-\frac{\pi \sqrt{2}}{\Gamma(3 / 4)} n^{3 / 4}+1+O\left(n^{-1 / 4}\right) .
\end{aligned}
$$

(Similar forms have been computed by the authors of [74] using somewhat heavier recurrence manipulations.) Here moments result effortlessly from taking partial derivatives of the BGF $H(x, 1, z)$ at $x=1$,

$$
\begin{aligned}
& H(x, 1, z)=\frac{1}{\delta^{1 / 4}}, \quad H_{x}^{\prime}(x, 1, z)=\frac{1}{\delta^{5 / 4}}-\frac{1}{\delta}, \\
& H_{x x}^{\prime \prime}(x, 1, z)=5 \delta^{-9 / 4}-8 \delta^{-2}+4 \delta^{-7 / 4}-5 \delta^{-5 / 4}+4 \delta^{-1},
\end{aligned}
$$

where $\delta=1-4 z$, upon extracting coefficients. As a consequence of the last equation, the variance also admits an explicit form involving binomial coefficients of fractional index, and its asymptotic value is

$$
\frac{2}{3} \pi \frac{8 \sqrt{2}-3 \pi}{\Gamma(3 / 4)^{2}} n^{3 / 2}-\frac{3 \pi \sqrt{2}}{\Gamma(3 / 4)} n^{3 / 4}+O(\sqrt{n}) .
$$

The number of balls of the second colour has thus been found to be of average order $n^{3 / 4}$, which coincides with the order of growth of the standard deviation. This is a strong indication of the presence of a non-Gaussian law (xiii) a property that we justify in the next subsection.

\subsection{Local limit laws}

The non-Gaussian character of the limit in triangular cases is obvious from the moment calculations of the previous subsection. It was observed by Smythe in [96]. However, apparently the martingale arguments of [96] do not lead to a determination of the limit law. Gouet [47] shows the existence of a limit distribution and remarks that "information on the moments of $Z$ [the limit] can be obtained from the difference equations characterizing the moments of $W_{n}\left[\right.$ our $\left.A_{n}\right]$ ", but he does not appear to have available a complete characterization of this limit.

Proposition 16 The random variable $A_{n}$ follows a local limit law whose density is expressed in terms of Mittag-Leffler functions. Precisely, for any compact set $I$ of $\mathbb{R}_{>0}$, and any $\xi$ in $I$ such that $\xi n^{\alpha / \sigma}$ is an integer, one has

$$
\mathbb{P}\left[A_{n}=a_{0}+\alpha \xi n^{\alpha / \sigma}\right]=\frac{1}{n^{\alpha / \sigma}} g(\xi)+O\left(\frac{1}{n^{2 \alpha / \sigma}}\right)
$$

\footnotetext{
(xiii) We are grateful to Dr Philippe Robert for asking us, in 2003, to determine the variance and the corresponding distribution, which eventually led to the developments of the present section, first reported in the 2003 version of [30] and in [92]
} 


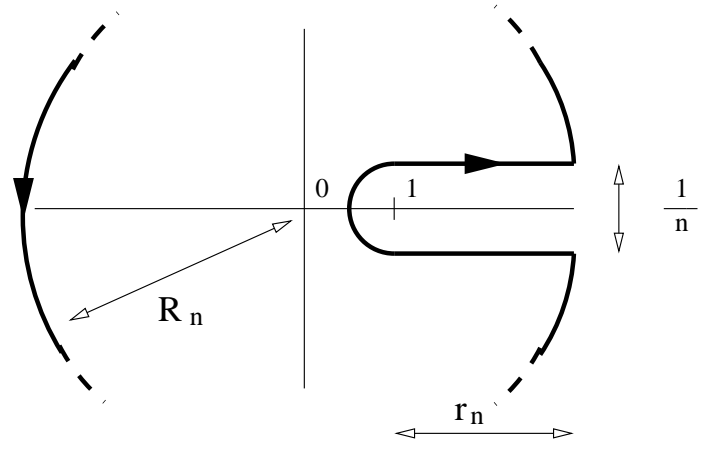

Figure 9: Hankel integration contour

where the error term holds uniformly with respect to $\xi \in I$. The function $g$ is defined on $\mathbb{R}_{>0}$ by means of the series expansion

$$
g(\xi)=\frac{\Gamma\left(\frac{t_{0}}{\sigma}\right)}{\Gamma\left(\frac{a_{0}}{\alpha}\right)} \xi^{a_{0} / \alpha-1} \sum_{k \geq 0} \frac{(-1)^{k}}{\Gamma\left(\frac{b_{0}-k \alpha}{\sigma}\right)} \frac{\xi^{k}}{k !} .
$$

Hence, $\quad \Gamma\left(\frac{a_{0}}{\alpha}\right) / \Gamma\left(\frac{t_{0}}{\alpha}\right)(-\xi)^{1-a_{0} / \alpha} g(-\xi) \quad$ is the inverse Laplace transform of a Mittag-Leffler function with parameters $\left(-\alpha / \sigma, b_{0} / \sigma\right)$. If $b_{0}=0$, it is exactly the density of a stable law of index $\alpha / \sigma$.

Proof: In connection with the analytic inclination of this paper, it is of interest to note that the stable-like distributions arise here for a bivariate generating function whose singular structure experiences a discontinuity at 1 (see [38, Ch. IX] for a perspective). The type of phenomenon encountered here resorts to the so-called critical composition scheme of [7]: this is described by a BGF of the form $F(u G(z))$ in the case where $F, G$ have algebraic singularities, while $G$ at its singularity assumes a value that creates a singularity for $F$. The result thus follows from [7], but for completeness, we sketch the proof here.

By expanding the outer radical $(\cdot)^{-a_{0} / a}$ in the expression (74) of $H$, one finds

$$
\mathbb{P}\left[A_{n}=a_{0}+k \alpha\right]=\frac{\Gamma(n+1) \Gamma\left(\frac{t_{0}}{\sigma}\right)\left(\begin{array}{c}
k+\frac{a_{0}}{\alpha}-1 \\
k
\end{array}\right)}{\sigma^{n} \Gamma\left(\frac{t_{0}}{\sigma}+n\right)}\left[z^{n}\right](1-\sigma z)^{-\frac{b_{0}}{\sigma}}\left(1-(1-\sigma z)^{\frac{\alpha}{\sigma}}\right)^{k} .
$$

The coefficient of $z^{n}$ is estimated by means of Cauchy's coefficient formula,

$$
\left[z^{n}\right](1-\sigma z)^{-b_{0} / \sigma}\left(1-(1-\sigma z)^{\alpha / \sigma}\right)^{k}=\frac{\sigma^{n}}{2 i \pi} \int_{\gamma}(1-z)^{-\frac{b_{0}}{\sigma}}\left(1-(1-z)^{\frac{\alpha}{\sigma}}\right)^{k} \frac{d z}{z^{n+1}}
$$

using a contour of integration $\gamma$ that winds around 1 and is scaled according to the general principles of singularity analysis [34, 38]. The contour is displayed in Figure 9 we choose $r_{n}=1+\mathrm{Cn}^{-\alpha / \sigma}$ and then, $R_{n}=\sqrt{r_{n}^{2}+(1 / n)^{2}}$, where $C$ is a fixed positive real. Set $k=\xi n^{\alpha / \sigma}$, where $\xi$ lies in a fixed compact subset $I$ of $\mathbb{R}_{>0}$. When $C$ is chosen sufficiently large $(C>\max (I)$ is enough), the contribution along the large circle is exponentially small for any $\xi$ in $I$. Setting $z=(1-t / n)$ then leads to the approximation,

$$
\mathbb{P}\left[A_{n}=a_{0}+k a\right] \sim \frac{\Gamma\left(\frac{t_{0}}{s}\right)}{\Gamma\left(\frac{a_{0}}{a}\right)} \xi^{a_{0} / a-1} \frac{1}{2 \pi n^{\alpha / \sigma}} \int_{\mathcal{H}} t^{-b_{0} / \sigma} e^{-\xi t^{\alpha / \sigma}+t} d t
$$

where $\mathcal{H}$ is a clockwise oriented loop around the negative real axis. Next, keep the kernel $t^{-b_{0} / \sigma} e^{t}$ and expand the other exponential; this gives us

$$
\begin{aligned}
\frac{1}{2 i \pi} \int_{\mathcal{H}} t^{-b_{0} / \sigma} e^{-\xi t^{\alpha / \sigma}+t} d t & =\frac{1}{2 i \pi} \sum_{k=0}^{\infty} \int_{\mathcal{H}} \frac{(-\xi)^{k}}{k !} t^{\frac{k a-b_{0}}{s}} e^{t} d t \\
& =\frac{1}{2 i \pi} \sum_{k=0}^{\infty} \frac{(-\xi)^{k}}{\Gamma\left(\frac{k a-b_{0}}{s}\right) k !}
\end{aligned}
$$

where the last equality derives from Hankel's classical representation of the Gamma function [103]. The error estimates are obtained by expanding the integral to one more term. The connection with stable laws 

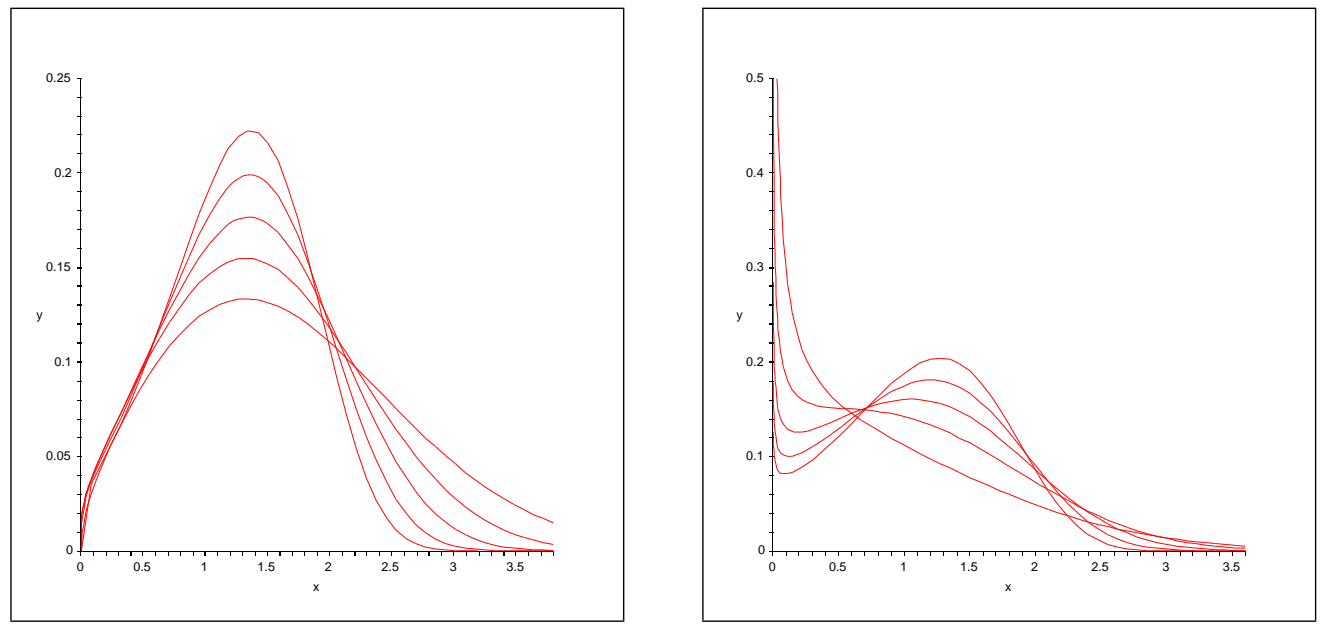

Figure 10: Limit laws of triangular urn models with 2 types of balls.

finally results from a trite comparison with the classical expression of their densities as found in the books of Feller [27] or Zolotarev [107].

Figure 10 displays some of the limit densities of triangular urns for various initial conditions. The plot on the left corresponds to limit laws of $\left[\begin{array}{cc}\alpha & 2 \\ 0 & \alpha+2\end{array}\right]$ urns, $\alpha \in[2 \ldots 6]$, with initial conditions $(\alpha+1,1)$, the plot on the right displays densities of the same urns, with initial conditions $(\alpha-1,1)$. Graphs with higher peaks correspond to higher values of $\alpha$.

\subsection{Mellin transforms and moments of fractional orders}

We finally determine the Mellin transform of the limit law of $A_{n}$. Recall that, given a function $h(\xi)$ defined over $\mathbb{R}_{\geq 0}$, its Mellin transform is defined by

$$
h^{\star}(s)=\int_{0}^{\infty} h(\xi) \xi^{s-1} d \xi .
$$

In particular, if $h(\xi)$ is the density of a nonnegative random variable $Y$, then $h^{\star}(s)=\mathbb{E}\left[Y^{s-1}\right]$. In other words, a Mellin transform provides moments of fractional (or even complex) orders, and its expression must extrapolate the values of the classical power moments of integral order.

Proposition 17 The density $g(x)$ of the limit law of $A_{n}$ admits a Mellin transform given, for any $s$ such that $\Re(s)>1-a_{0} / \alpha$, by

$$
g^{*}(s)=\frac{\Gamma\left(\frac{a_{0}+(s-1) \alpha}{\alpha}\right) \Gamma\left(\frac{t_{0}}{\sigma}\right)}{\Gamma\left(\frac{a_{0}}{\alpha}\right) \Gamma\left(\frac{t_{0}+(s-1) \alpha}{\sigma}\right)} .
$$

The moment of order $\kappa$ of the limit law exists for any complex $\kappa$ with $\Re(\kappa)>-a_{0} / a$ and is equal to $g^{*}(\kappa+1)$, with $g^{\star}$ provided by 81 .

Proof: The Mellin transform of the density is given by

$$
g^{*}(s)=\int_{0}^{+\infty} \xi^{s-1}\left(\frac{\Gamma\left(\frac{t_{0}}{\sigma}\right)}{\Gamma\left(\frac{a_{0}}{\alpha}\right)} \xi^{a_{0} / \alpha-1} \int_{\mathcal{H}} t^{-b_{0} / \sigma} e^{-\xi t^{\alpha / \sigma}+t} d t\right) d \xi
$$

where $\mathcal{H}$ is a clockwise oriented loop around the negative real axis. Define

$$
F(\xi, t)=\frac{\Gamma\left(\frac{t_{0}}{\sigma}\right)}{\Gamma\left(\frac{a_{0}}{\alpha}\right)} \xi^{a_{0} / \alpha+\sigma-2} t^{-b_{0} / \sigma} e^{-\xi t^{\alpha / \sigma}+t} .
$$

The integral $\int_{0}^{+\infty} \xi^{s-1} e^{-\xi \gamma} d \xi$ converges for any $\lambda$ such that $\Re(\lambda)>0$ and is equal to $\lambda^{-s} \Gamma(s)$. The contour $\mathcal{H}$ can is chosen as displayed below, 


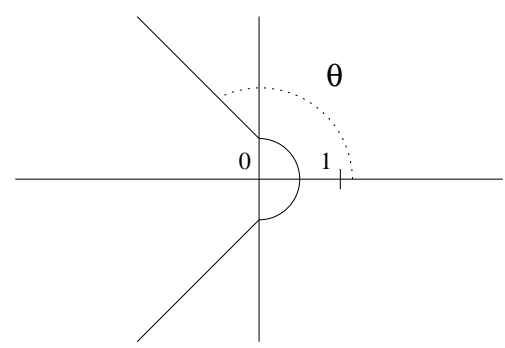

with $\frac{\pi}{2}<\theta<\frac{\sigma}{\alpha} \frac{\pi}{2}$. Given this integration contour, for any $t$ of $\mathcal{H}$ and any $s$ satisfying $\Re(s)>1-a_{0} / \alpha$, the integral $\int_{0}^{+\infty} F(\xi, t) d \xi$ converges and its value is

$$
\int_{0}^{+\infty} F(\xi, t) d \xi=\Gamma\left(\frac{a_{0}+(s-1) \alpha}{\alpha}\right) t^{-\left(t_{0}+(s-1) \alpha\right) / \sigma} e^{t} .
$$

It suffices to integrate this expression with respect to $t$, and the statement follows.

\section{Intermezzo: the OK Corral urn $[0,-1,-1,0]$}

The analytic approach to $2 \times 2$ urn models leads to the solution of several classical problems in a unified way. Instances that have been encountered in earlier sections include the coupon collector's problem, the Ehrenfest model, records and rises in permutations, leaves in binary increasing trees and trees of acquaintance networks, as well as the fringe analysis of 2-3 trees. We briefly discuss here results obtained in the period 2004-2005 by two of us [36], which will give rise to a separate publication. These results exemplify further the interest of an analytic approach to urn models, as well as the diversity of stochastic behaviours that such models may lead to.

The model at stake here is described by a matrix

$$
\mathcal{M}=\left(\begin{array}{cc}
0 & -1 \\
-1 & 0
\end{array}\right)
$$

which is not of a type hitherto considered (here, $\beta=\gamma=-1$, while all previous results are based on the assumption $\beta, \gamma \geq 0$ ). If an $\times$ ball is picked up, then a y ball should be taken out of the urn; if a $\mathrm{y}$ ball is picked up, then $\mathrm{a} \times$ ball should be taken out of the urn. The process stops once balls of one or the other colour are exhausted.

A vivid interpretation of the urn defined by 82 is as follows. Consider two groups of gunwomen facing each other. At time 0 , the first group comprises $m$ fighters and the second group comprises $n$ fighters. (We assume throughout $m, n \geq 1$.) At each discrete instant, a fighter chosen uniformly at random shoots and kills a member of the opposite group. The problem consists in quantifying the chances for a team to win as well as the number of survivors. In other words: To what extent does a numerical advantage in the field increase the chances of a favorable outcome? As pointed out by Kingman [69], this question is a basic one in the mathematical theory of warfare and conflicts initiated by Frederick William Lanchester (1868-1946).

The problem of analysing the urn $(82)$ has been named the "OK Corral Problem" by reference to the famous gunfight at the OK Corral Ranch of Tombstone in 1881. It makes sense both in the "fair" case where $m, n$ are equal (or nearly equal) and in the "unfair" case where $m \gg n$ or $n \gg m$. It was introduced by Williams and McIlroy in [105]. These authors gave a heuristic argument based on a deterministic differential system and a diffusion process approximation suggesting for the mean number $\mu(m, n)$ of survivors the asymptotic estimates

$$
\left\{\begin{aligned}
\mu(m, n) & \sim \sqrt{m^{2}-n^{2}}, & & \text { for } m \gg n, \\
\mu(m, m) & \sim 2 K m^{3 / 4}, & & \text { with } K:=3^{-1 / 4} \pi^{-1 / 2} \Gamma(3 / 4) .
\end{aligned}\right.
$$

In particular the numerical evidence for the correctness of the second estimate was overwhelming.

Kingman [68] proved the estimate of $\mu(m, m)$ in 83 and a good deal more. By recurrence manipulations, he obtained, for the fair case $m=n$, the asymptotic form of higher moments of the number $S$ of survivors, which leads to a characterization of the limit distribution of $S$ :

$$
\mathbb{P}\left(S \leq \lambda n^{3 / 4}\right) \rightarrow \sqrt{\frac{2}{\pi}} \int_{0}^{\lambda^{2} / 3} e^{-x^{2} / 2} d x .
$$



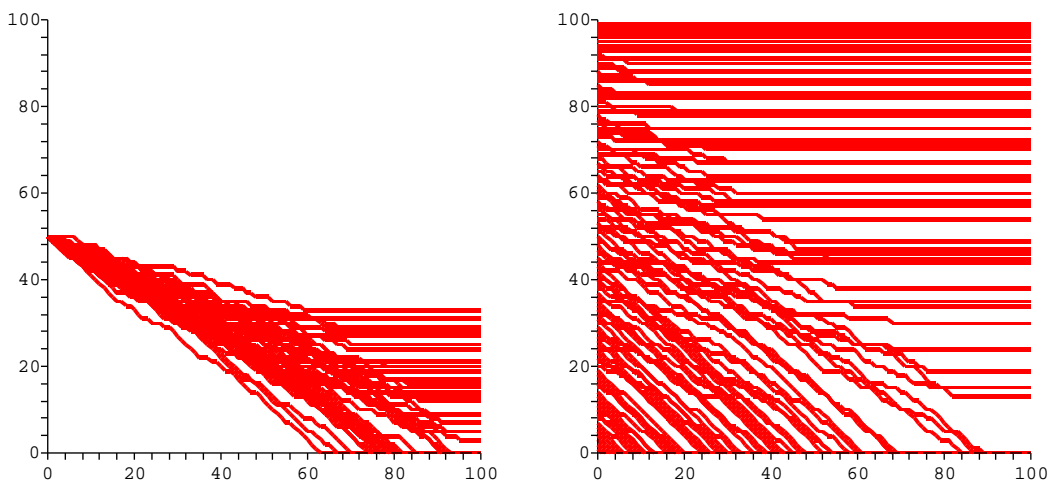

Figure 11: Left: Fifty random evolutions in the fair case $m=n=50$. Right: Random evolutions in the general case $m+n=100$, with $m \in[1 \ldots 99]$. The horizontal axis represents time, the vertical axis corresponds to the size of the first group.

Remarkably, the methods of [68] also make it possible to justify the first approximation of [83] for the unfair fight, in the case when $m-n$ is large compared to $\sqrt{m}$. Finally Theorem 3 of $[68]$ describes the transition region when $m-n \sim x \sqrt{m}$ in terms of a Gaussian error function (erf). Kingman's arguments in [68] are based on moment methods, and accordingly they say little regarding speed of convergence to the asymptotic limit in estimates like [84]. This problem is revisited by Kingman and Volkov in [70] who proved a local limit law, (i.e., convergence to the Gaussian density function) accompanied with error bounds for the fair case $m=n$, in a form consistent with [84). The results of [70] are based on ingenious coupling arguments. Interestingly enough, they also lead to an exact expression involving a double alternating sums for the probabilities of interest.

The analytic results reported in [36] (see also [92] for a preliminary presentation) are the following. First, it is possible to obtain expressions for the probability distribution of the number of survivors that are valid in all (fair/unfair) cases and are simpler than those of Kingman and Volkov, as they involve only a single alternating sum. Then, an explicit single alternating sum representation for the probability of survival of the first group, can be derived. The approach reveals a connection with Eulerian numbers and it provides an independent proof of Kingman's phase transition (84) as well as a precise and optimal speed of convergence estimate. If the ratio $\alpha=m / n$ satisfies $\alpha<1$, then the probability for the first group to win becomes exponentially small, and one can precisely quantify this exponential smallness. Precise statements are given now.

Proposition 18 (OK Corral: Exact forms) (i) The probability $\mathbb{P}_{\searrow}(m, n, s)$ of observing s survivors from the first group in the OK Corral problem admits the exact expressions:

$$
\begin{aligned}
\mathbb{P}_{\searrow}(m, n, s) & =\frac{s !}{(m+n) !} \sum_{k=1}^{m}(-1)^{m-k}\left(\begin{array}{c}
k-1 \\
s-1
\end{array}\right)\left(\begin{array}{c}
m+n \\
n+k
\end{array}\right) k^{m+n-s} \\
& =\frac{s !}{(m+n) !} \sum_{k=1}^{n}(-1)^{n-k}\left(\begin{array}{c}
k+s-1 \\
k
\end{array}\right)\left(\begin{array}{c}
m+n \\
m+k
\end{array}\right) k^{m+n-s} .
\end{aligned}
$$

(ii) The probability $\mathbb{P}_{1}(m, n)$ of survival of the first group satisfies

$$
\begin{aligned}
\mathbb{P}_{1}(m, n) & =\frac{1}{(m+n) !} \sum_{k=1}^{n}(-1)^{m-k}\left(\begin{array}{c}
m+n \\
n+k
\end{array}\right) k^{m+n} \\
& =\frac{1}{(m+n) !} \sum_{k=1}^{m} A_{m+n, k},
\end{aligned}
$$

where $A_{n, k}=\sum_{0 \leq j \leq k}(-1)^{j}\left(\begin{array}{c}n+1 \\ j\end{array}\right)(k-j)^{n}$. In particular, the survival probability is equal to the probability that a random permutation of size $m+n$ has less than $m$ rises.

Proof (sketch): $(i)$ The starting point is the time-reversal transformation of Kingman and Volkov [70] (see Note 2. p. 66 for background): it is possible to view histories of the OK Corral process as mirror images 


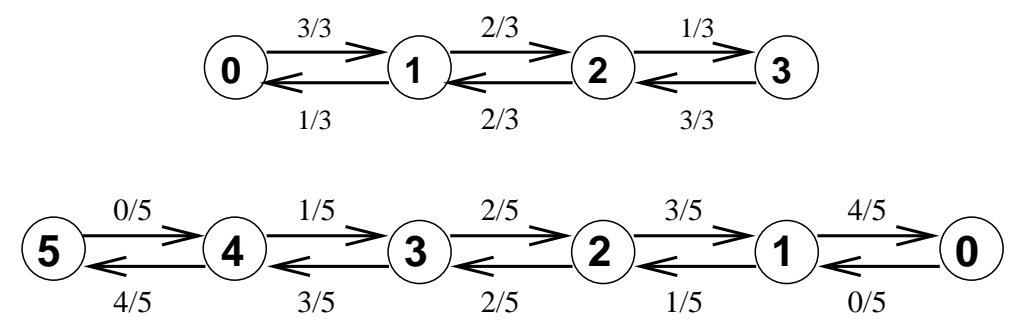

Figure 12: The transition graphs of the Ehrenfest urn with $N=3$ particles (top) and of the Mabinogion urn with $N+2=5$ sheep (bottom) illustrate the duality of the two models, under time reversal.

of histories of Friedman's urn, up to a normalizing factor. It then becomes possible to make use of the complete analytic solution of Friedman's urn as given by [20, p. 68. Standard expansions provide the first form stated in 85 , while the seemingly similar second form results from a suitable integral representation. (ii) Generating function calculations make it possible to carry explicitly the summation needed to derive the evaluation 86 from that of 85 .

Proposition 19 (OK Corral: asymptotic forms) ( $i$ ) Consider nearly fair fights defined by initial conditions $m, n$ satisfying $\frac{m-n}{\sqrt{m+n}}=\theta$, where $\theta$ lies in a compact interval of the real line. Then, the survival probability of the first group satisfies

$$
\mathbb{P}_{1}(m, n)=\frac{1}{\sqrt{2 \pi}} \int_{-\infty}^{\theta \sqrt{3}} e^{-t^{2} / 2} d t+O\left(\frac{1}{n}\right)
$$

the uniform error term $O\left(n^{-1}\right)$ being optimal.

(ii) Consider the "unfair" regime $m=\alpha$, with $\alpha<1$, a constant. There exist a function $W(\alpha)$ satisfying $W(0)=-\infty, W(1)=0$, such that the probability of survival of the first group satisfies

$$
\frac{1}{n} \log \mathbb{P}_{1}(\alpha n, n)=W(\alpha)+O\left(\frac{\log n}{n}\right) .
$$

The function $W$ is defined for $\alpha \in(0,1)$ by $W(\alpha)=-(1+\alpha) \log \left(\frac{\log x_{\alpha}}{x_{\alpha}-1}\right)-\alpha \log x_{\alpha}$, the real number $x_{\alpha}$ being defined implicitly by the equation $\frac{x_{\alpha}}{x_{\alpha}-1}-\frac{1}{\log x_{\alpha}}=\frac{\alpha}{1+\alpha}$.

Proof (sketch): $(i)$ The estimate $(88)$ is a consequence of 86 , after a central limit estimate with speed is developed, based on quasi-powers approximation. ( $i i)$ The estimate 89 , is a large deviation estimate established by a process similar to the one detailed earlier in the case of semi-sacrificial urns.

Other statements of [36] include exact representations for all power moments of the number of survivors in the fair case $m=n$ : these make precise and complement calculations of Kingman (who obtained moments of orders $4,8,12, \ldots$ ) and lead to another proof of (84). In particular, one gains direct access to the mean itself, whose expression is a sum that involves the birthday paradox function,

$$
Q(k):=\frac{k}{k}+\frac{k(k-1)}{k^{2}}+\cdots+\frac{k !}{k^{k}} .
$$

An integral representation of the Nörlund-Rice type [37] then leads to an asymptotic expansion of the mean number of survivors. It is of interest to note that, in this perspective, the asymptotic analysis relies on an analytic extrapolation of the sequence $(Q(k))$ to the complex plane.

Note 10. The Mabinogion urn. The time reversal transformation that relates the OK Corral urn to Friedman's can be of interest in other contexts. For instance, the Mabinogion urr (xiv) described by Williams in [104 p. 159] and corresponding to the matrix

$$
\mathcal{M}=\left(\begin{array}{cc}
1 & -1 \\
-1 & 1
\end{array}\right)
$$

analytically reduces to the Ehrenfest urn when time is reversed: see Figure 12 (details are omitted in this short abstract). $\square$

(xiv) According to a medieval Welsh folk tale, the Mabinogion, there is a magical flock of sheep, some black, some white. Whenever a black sheep bleats, a white sheep becomes instantly black; whenever a white sheep bleats, a black sheep becomes instantly white. 


\section{Higher dimensional models}

We briefly discuss here a few additional urn models involving balls of more than two colours. Let $m$ be the number of colours. We now let $x_{j}$ be the formal variable associated to colour $j$. When there are only three types of balls ( $m=3$ ), we tend to use $x:=x_{1}, y:=x_{2}$, and $u:=x_{3}$, for readability.

Definition 5 For an urn with matrix

$$
\mathcal{M}=\left(M_{i, j}\right),
$$

the associated differential system is the following collection of $m$ ordinary differential equation:

$$
\Sigma: \quad\left\{\dot{x}_{i}=x_{i} \prod_{j=1}^{m} x_{j}^{M_{i, j}}\right\}_{i=1 \ldots m},
$$

with initial conditions

$$
\left\{x_{i}(0)=x_{i .0}\right\}_{i=1 \ldots m} .
$$

By a slight abuse of notation, the system (91) can be rewritten as

$$
\dot{\mathbf{X}}=\mathbf{X}^{I+M},
$$

with $I$ the identity matrix of the proper dimension. An analogue of Theorem 1 still holds for the associated system.

Theorem 6 (Basic isomorphism, $m \times m$ urns) Consider an $m \times m$ urn with matrix $\mathcal{M}$ that is balanced, that is, $\sum_{j} M_{i, j}=\sigma$ for all $j=1 \ldots m$. Let $\mathbf{X}(t)=\left(X_{i}(t)\right)_{i=1}^{m}$ be the solution of the system (91) under the initial conditions (92). Assume that $\prod_{i=1}^{m} x_{i, 0} \neq 0$. Then, there exists a small neighbourhood of 0 in the z-plane such that

$$
H\left(x_{1,0}, \ldots, x_{m, 0}, z\right)=\prod_{i=1}^{m} X_{j}(t)^{a_{i, 0}},
$$

when the urn is initialized with $a_{i, 0}$ balls of colour $i$.

In general, for $m \geq 3$, we do not know of first integrals for this system (see Note 11 below). Accordingly, our discussion in this article will be limited to special cases, ones that usually involve symmetry. Fortunately, urn models naturally occurring in real life often possess some interesting structure—often, precisely, a symmetry of sorts.

Note 11. Nonintegrability ${ }^{(\mathrm{xv})}$ The system

$$
\dot{x}=y^{2}, \quad \dot{y}=u^{2}, \quad \dot{u}=x^{2},
$$

together with its $d$ dimensional generalization, has been considered by Jouanolou in [65] in the context of Pfaffian systems. (This model does have combinatorial content since it corresponds to analysing the depth of nodes in binary search trees and increasing trees taken modulo $d$; it is thus a natural generalization of the generation-parity urn, Example 4) A theorem of [65] asserts the non-existence of a polynomial first integral for 93]. Elementary proofs of non-integrability (in the sense that they are based on basic differential algebra rather than algebraic geometry) are discussed in [82, 86]. These results are of great interest: they imply that the global approach that we developed for $2 \times 2$ urns (based on the first integral of Section 3) admits of no universal generalization in any dimension $d \geq 3$.

\subsection{The purely autistic (hyper-Pólya) urn}

Nobody cares about her neighbour: the matrix is the unit matrix, which for $m=3$ is

$$
\mathcal{M}=\left(\begin{array}{lll}
1 & 0 & 0 \\
0 & 1 & 0 \\
0 & 0 & 1
\end{array}\right)
$$

This is a generalization of the original Pólya urn. For $m=3$, still, the associated differential system reads

$$
\dot{x}=x^{2}, \quad \dot{y}=y^{2}, \quad \dot{u}=u^{2} .
$$

\footnotetext{
(xv) We are greatly indebted to Jacques-Arthur Weil and Aleksandar Sedoglavić for insightful discussions and references relative to these questions. In particular A. Sedoglavić has mostly kindly provided the references to the works of Jouanolou, Moulin-Ollagnier, $\begin{array}{lllll}\text { Nowicki et al } & 65 & 82 & 86\end{array}$.
} 

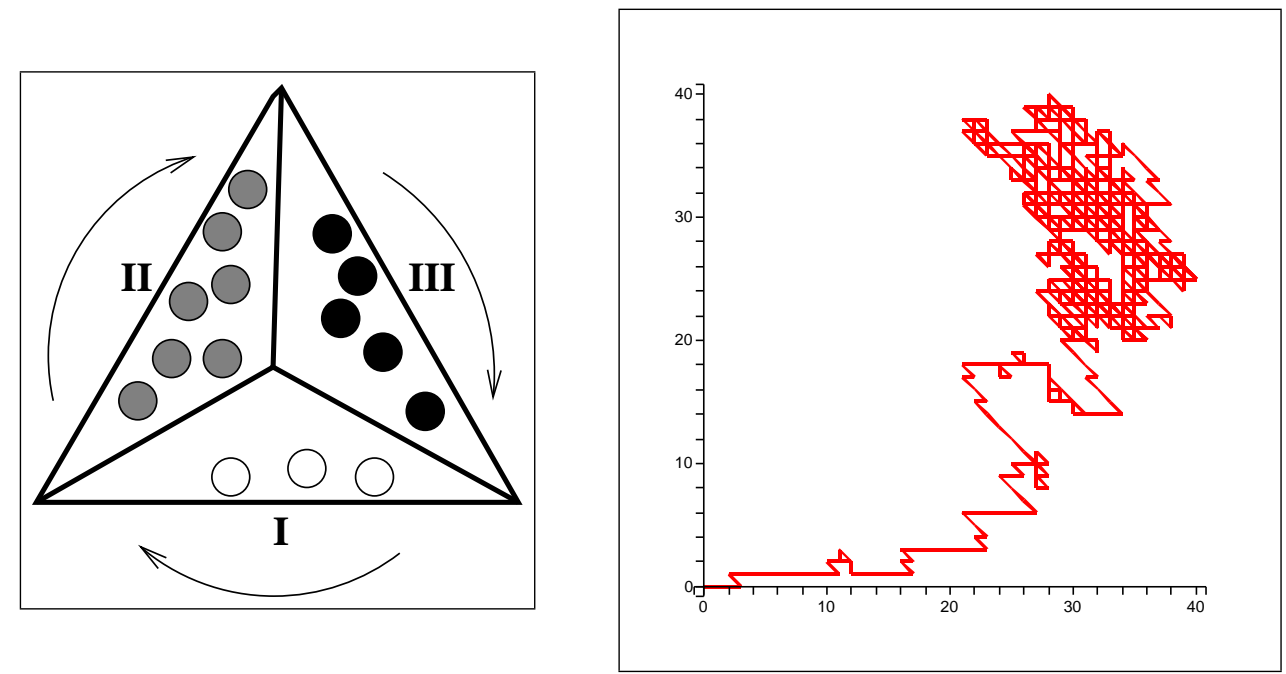

Figure 13: The cyclic chambers urn with $m=3$ chambers. Left: overall structure. Right: a random evolution of the urn initialized with $\left(a_{0}, b_{0}, c_{0}\right)=(90,0,0)$ represented by the successive values of $\left(B_{n}, C_{n}\right)$ for $n=0 \ldots 900$.

Not surprisingly, variables separate. Taking into account the initial condition vector $\left(x_{0}, y_{0}, u_{0}\right)$, we have

$$
x(t)=\frac{x_{0}}{1-x_{0} t}, \quad y(t)=\frac{y_{0}}{1-y_{0} t}, \quad u(t)=\frac{u_{0}}{1-u_{0} t} .
$$

The complete GF of urn histories when starting from a configuration of composition $\left(a_{0}, b_{0}, c_{0}\right)$ is then

$$
H\left(x_{0}, y_{0}, u_{0}, z\right)=\left(\frac{x_{0}}{1-x_{0} z}\right)^{a_{0}}\left(\frac{y_{0}}{1-y_{0} z}\right)^{b_{0}}\left(\frac{u_{0}}{1-u_{0} z}\right)^{c_{0}}
$$

Coefficient extraction is immediate, and we get by the binomial theorem:

Proposition 20 For the purely autistic urn in dimension $m=3$ initialized with $\left(a_{0}, b_{0}, c_{0}\right)$, one has

$$
\mathbb{P}\left(A_{n}=a, B_{n}=b, C_{n}=c\right)=\frac{\left(\begin{array}{c}
a-1 \\
a_{0}-1
\end{array}\right)\left(\begin{array}{c}
b-1 \\
b_{0}-1
\end{array}\right)\left(\begin{array}{c}
c-1 \\
c_{0}-1
\end{array}\right)}{\left(\begin{array}{c}
a_{0}+b_{0}+c_{0}+n-1 \\
a_{0}+b_{0}+c_{0}-1
\end{array}\right)},
$$

assuming $a+b+c=n+a_{0}+b_{0}+c_{0}$.

This formula generalizes the case $m=2$; see (18). It is of course easily derived directly (in combinatorial terms, we are talking of a shuffle of histories corresponding to each one of the various colours, and shuffles translate into products of exponential generating functions), extends to any dimension $m$, and is very well known.

\subsection{The cyclic chambers (hyper-Ehrenfest) urn}

This is a natural generalization of the Ehrenfest urn introduced for dimension $m=2$ in $\$ 2.3$. p. 69. For $m=$ 3 , imagine that you have three chambers arranged cyclically at third roots of unity. Particles are allowed to migrate freely from one chamber to the next in clockwise order (Figure 13, left). (Thus the model has a privileged direction for the migration of particles.) The matrix that models the system is

$$
\mathcal{M}=\left(\begin{array}{ccc}
-1 & 1 & 0 \\
0 & -1 & 1 \\
1 & 0 & -1
\end{array}\right)
$$

(Each particle becomes a ball whose colour represents the chamber the particle is currently in.)

The associated differential system is then

$$
\Sigma: \quad \dot{x}=y, \quad \dot{y}=u, \quad \dot{u}=x,
$$

so that $x(t)$ satisfies

$$
\frac{d^{3}}{d t^{3}} x(t)=x(t)
$$


which implies that $x(t)$ is a linear combination of

$$
e^{t}, \quad e^{\omega t}, \quad e^{\omega^{2} t}, \quad \text { with } \quad \omega:=e^{2 i \pi / 3} .
$$

And similarly for $y(t), u(t)$.

The analysis is best developed for the general case of dimension $m$. Define the sectioned exponentials:

$$
E_{r, m}(t):=\sum_{n \equiv r \bmod m} \frac{t^{n}}{n !},
$$

so that $E_{r, m}(t)=O\left(t^{r}\right)$ as $t \rightarrow 0$. For instance: $E_{0,2}(t)=\cosh t$ and $E_{1,2}(t)=\sinh t$. Equivalently, we have

$$
E_{r, m}(t)=\frac{1}{m} \sum_{j=1}^{m} \omega^{-j r} e^{\omega^{j} t}, \quad \omega:=\exp \left(\frac{2 \pi i}{m}\right) .
$$

These functions with $j=0, \ldots, m-1$ clearly form a basis of the linear space of solutions of the equation

$$
\frac{d^{m}}{d t^{m}} f(t)-f(t)=0
$$

The system $\Sigma$ with initial conditions $\mathbf{x}_{0}=\left(x_{1,0}, x_{2,0}, \ldots\right)$ then has solutions

$$
X_{1}\left(t \mid \mathbf{x}_{0}\right)=\sum_{j=1}^{m} x_{j, m} E_{j-1, m}(t)
$$

and similarly for the other $X_{j}$ 's (with a cyclic shift of the initial condition vector $\left(x_{1,0}, x_{2,0}, \ldots\right)$. This provides the complete analytic solution of the urn model:

Proposition 21 For the cyclic chambers urn in dimension $m$ initialized with a vector $\left(a_{1,0}, a_{2,0}, \ldots\right)$, the complete GF of histories satisfies

$$
H\left(\mathbf{x}_{0} ; z\right)=\prod_{j=1}^{m} X_{j}\left(z \mid \mathbf{x}_{\mathbf{0}}\right)^{a_{j, 0}}
$$

where

$$
X_{j}\left(z \mid \mathbf{x}_{0}\right)=\sum_{r=1}^{m} x_{j+r-1, m} E_{j-1, m}(t),
$$

in which we interpret $x_{m+1} \equiv x_{1}, x_{m+2} \equiv x_{2}$, and so on. In particular, the probability that an urn initialized with $(N, 0,0)$ returns to its original state in $n$ steps is

$$
\mathbb{P}\left(A_{n}=N\right)=\frac{n !}{N^{n}}\left[z^{n}\right] E_{0, m}(z)^{N} .
$$

Speed of convergence to equilibrium. Let us first return to $m=3$ and specialize the discussion to a system initialized with $N$ balls of the first colour and no ball of any of the other colours. The initial composition vector is then $(N, 0,0)$. Let $h_{n}$ be the number of histories corresponding to the event that at time $n$, balls are gain all of the first colour, i.e., all particles have returned home. The EGF of the sequence $h_{n}$ is by 95 :

$$
h(z)=E_{0,3}(z)^{N} .
$$

The multinomial expansion then provides

$$
h(z)=\frac{1}{3^{N}} \sum_{j_{1}+j_{2}+j_{3}=N}\left(\begin{array}{c}
N \\
j_{1}, j_{2}, j_{3}
\end{array}\right) \exp \left(\left(j_{1}+\omega j_{2}+\omega^{2} j_{3}\right) z\right),
$$

so that

$$
h_{n}=\frac{1}{3^{N}} \sum_{j_{1}+j_{2}+j_{3}=N}\left(\begin{array}{c}
N \\
j_{1}, j_{2}, j_{3}
\end{array}\right)\left(j_{1}+\omega j_{2}+\omega^{2} j_{3}\right)^{N} .
$$

(This quantity is nonzero only if $n \equiv 0 \bmod 3$.) The expression above is seen to nicely extend the analysis of the standard Ehrenfest model. 


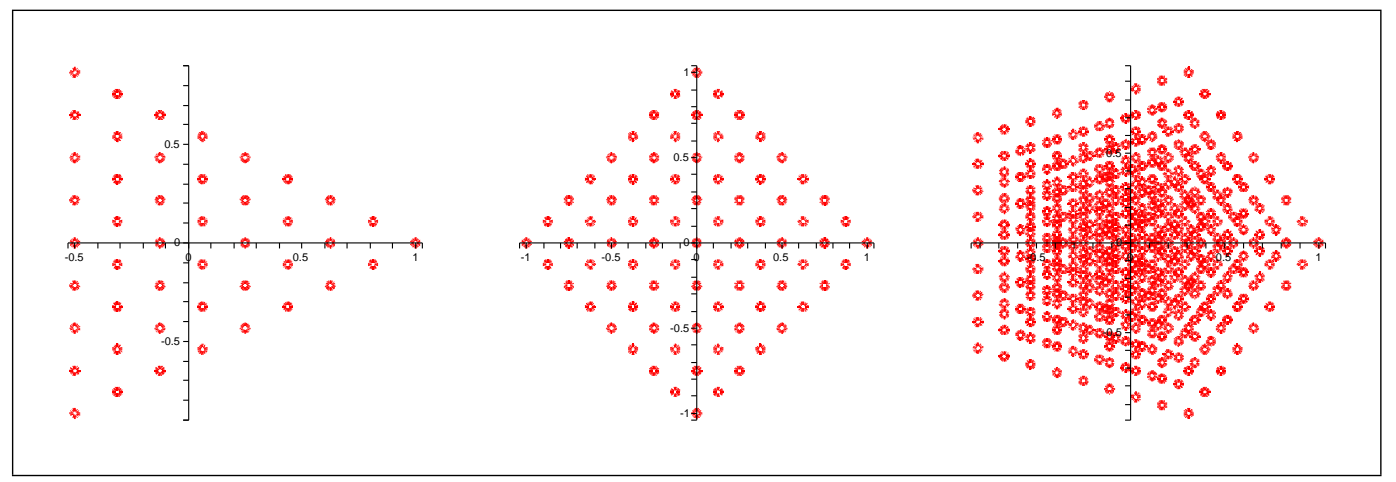

Figure 14: The spectrum of the Markov chain describing the cyclic $m$-chambers (hyper-Ehrenfest) urn, when $m=$ $3,4,5$.

The system is, like in dimension 2, represented probabilistically by finite Markov chains-the complete one (analogous to the hypercube) and the reduced one (see $\$ 2.3$ and Figure 5 p. 71). The reduced chain, which keeps track only of the population of each chamber, has $\left(\begin{array}{c}N+2 \\ 2\end{array}\right)$ states and a transition matrix of the same dimension. The spectrum of that matrix is also visible on an expression (96): the eigenvalues are precisely the quantities

$$
\lambda_{j_{1}, j_{2}}=\frac{j_{1}}{N}+\frac{j_{2}}{N} \omega+\left(1-\frac{j_{1}}{N}-\frac{j_{2}}{N}\right) \omega^{2} .
$$

The reason why the enumerative result $(96$ "contains" all eigenvalues is the following. For any directed graph, the number of closed paths with a root vertex distinguished having length $n$ is $\operatorname{Tr} A^{n}$ with $A$ the adjacency matrix of the graph and Tr the trace operator-this quantity is none other than the $n$th power sum symmetric function of the eigenvalues (with possible multiplicities): see for instance [11, p. 12] or [38, Ch. V]. For the graph of the complete Markov chain (with $3^{N}$ states, where the identities of the balls are preserved, see the discussion of $\$ 2.3$, the number of closed paths is equal to $3^{N} h_{n}$, by the fundamental symmetry of the model. Since the reduced chain is obtained by aggregating states of the complete chain, the sets of eigenvalues of both matrices are the same, only multiplicities differ. Figure 14(left) displays the corresponding eigenvalues when $m=3$ and $N=8$.

The second largest eigenvalues dictate the speed of convergence to equilibrium. In Figure 13 (right), we see a random trajectory represented by the pair $\left(B_{n}, C_{n}\right)$ of the urn initialized with $(N, 0,0)$ : the urn first evolves by transferring balls from Chamber I to Chamber II [the nearly horizontal part of the path in Figure 13], then enough balls migrate from Chamber II to Chamber III [the slanted zigzag part of the path], after which the urn tends to spend most of the time near the "equilibrium" value $(N / 3, N / 3)$ [the blob around the point $(30,30)]$. The common modulus of the second eigenvalues is made fully explicit by the calculation (97). These correspond to $j_{2}=1, j_{3}=0$ or $j_{2}=0, j_{3}=1$ so that their common modulus $\mu^{[3]}$ is

$$
\mu^{[3]}=\left|\left(1-\frac{1}{N}\right)+\frac{1}{N} e^{2 i \pi / 3}\right|=\sqrt{1-\frac{3}{N}+\frac{3}{N^{2}}} .
$$

In dimension $m$ (i.e., there are $m$ cyclically arranged chambers), this modulus is made fully explicit by a similar calculation.

Proposition 22 For the Markov chain associated to the cyclic chambers urn with $m$ cyclic chambers, the modulus of the second largest eigenvalues is

$$
\mu^{[m]}=\left|\left(1-\frac{1}{N}\right)+\frac{1}{N} e^{2 i \pi / m}\right|=\sqrt{\left(1-\frac{2}{N}\right)^{2}+\frac{4}{N}\left(1-\frac{1}{N}\right) \cos ^{2} \frac{\pi}{m}} .
$$

The formula (98) is also compatible with what we know of the standard Ehrenfest urn $(m=2)$, for which the spectrum is $N / N,(N-2) / N, \ldots,-(N-2) / N,-N / N$, so that the convergence to equilibrium is an exponential with rate $\mu^{[2]}=(N-2) / N$. The spectrum for $N=8$ and $m=3,4,5$ is represented in Figure 14. (It is unclear to us how Kac's treatment could be extended to cover such higher dimensional cases-for relevant techniques, see Mohar's survey [85].) 
Note 12. Combinatorial analysis of the cyclic chambers urn. The formulae above are not all that surprising from a combinatorial point of view: let the urn be, as above, initialized with $N$ balls of the first type and return to its initial state after $n$ moves. A corresponding history can be regarded as a partition of the set $\{1, \ldots, n\}$ into an ordered collection of $N$ (possibly empty) disjoint classes, each of which has size a multiple of 3: the $j$ th class records the various times at which ball $j$ has been active. Given general principles of combinatorial analysis, this makes it obvious why the generating function is an $N$ th power of a sectioned exponential. (This generalizes earlier observations of Flajolet, Françon, Goulden, and Jackson [14,29,50] relative to the case $m=2$.)

The differential system approach can also be extended to urns involving random transitions, provided that in all cases the model remains balanced. In that case the system becomes polynomial (instead of being plainly monomial). To illustrate this point, we briefly discuss an undirected version of the cyclic chambers model.

Example 9. The symmetrical three-chambers model. The case where each of three chambers communicates with its two neighbours can be modelled by a matrix of the form

$$
\mathcal{M}=\left(\begin{array}{ccc}
-1 & Z & 1-Z \\
Z & -1 & 1-Z \\
Z & 1-Z & -1
\end{array}\right),
$$

where the Bernoulli random variable $Z$ assumes the values 0,1 with equal probability, and each transition of the urn involves an independent draw of a variable of type $Z$. A simple extension of Theorem 6 then shows that one should consider the differential system

$$
\left\{\begin{array}{l}
\dot{x}=\frac{1}{2}(y+u) \\
\dot{y}=\frac{1}{2}(u+x) \\
\dot{u}=\frac{1}{2}(x+y)
\end{array}, \quad\left\{\begin{array}{l}
\dot{x}(0)=x_{0} \\
\dot{y}(0)=y_{0} \\
\dot{u}(0)=u_{0} .
\end{array}\right.\right.
$$

The sum $S:=x+y+u$ satisfies $\dot{S}=S$, so that

$$
S(t)=\left(x_{0}+y_{0}+z_{0}\right) e^{t}
$$

and $x(t)$ satisfies the differential equation $\dot{x}+\frac{1}{2} x=\frac{1}{2} S$. Thus, the first base function is

$$
X(z)=\frac{1}{3}\left(x_{0}+y_{0}+u_{0}\right) e^{z}+\frac{1}{3}\left(2 x_{0}-y_{0}-u_{0}\right) e^{-z / 2},
$$

and symmetrically for $Y(z), U(z)$. In particular, starting with the initial configuration $(N, 0,0)$, the probability that all particles have returned to the first chamber at time $n$, obtained by setting $y_{0}=u_{0}=0$ in $(100)$, is

$$
\mathbb{P}\left(A_{n}=N\right)=\frac{n !}{N^{n}}\left[z^{n}\right]\left(\frac{1}{3} e^{z}+\frac{2}{3} e^{-z / 2}\right)^{N} .
$$

For a fixed value of $N$, this quantity is, asymptotically in $n$,

$$
\mathbb{P}\left(A_{n}=N\right)=\frac{1}{3^{N}}\left[1+O\left(\left(1-\frac{1}{2 N}\right)^{n}\right)\right] .
$$

This last estimate reflects the fact that the symmetrical urn reaches equilibrium faster than its nonsymmetrical counterpart.

More generally, models of multichamber systems where communication between chambers is described by a finite graph equipped with probabilities [67] are amenable to such a treatment. Relations between urn models and interacting particle systems as considered by Itoh and his coauthors [59,60,61] seem to deserve further study.

\subsection{The generalized coupon collector's urn}

Start with the double coupon collector problem: Alice and her little brother Bob collect images or coupons found in chocolate tablets, and Alice who comes first passes all her duplicates to Bob. They want each to have a complete collection, knowing that there are initially $N$ different coupons. How much time do they need, on average, in probability?

The problem is modelled by the urn

$$
\mathcal{M}=\left(\begin{array}{rrr}
-1 & 1 & 0 \\
0 & -1 & 1 \\
0 & 0 & 0
\end{array}\right)
$$


Initially there are $N$ balls all of the first colour (x). As soon as an $\mathrm{x}$-ball has been picked up, it becomes a ball of the second colour (y). A y ball, when picked up, becomes of the third colour ( $u$ ) and remains so for ever. It is easily realized that the double coupon collector problem is adequately modelled in this way.

The associated differential system is

$$
\dot{x}=y, \quad \dot{y}=u, \quad \dot{u}=u \text {. }
$$

It is linear with constant coefficients and of a triangular form: one can then solve for $u$, which gives a simple exponential, then solve backwards by successive integrations. Given the initial condition vector $\left(x_{0}, y_{0}, u_{0}\right)$, the solution is

$$
U(z)=u_{0} e^{z}, \quad Y(z)=y_{0}+u_{0}\left(e^{z}-1\right), \quad X(z)=x_{0}+y_{0} z+u_{0}\left(e^{z}-1-z\right) .
$$

The complete GF of histories when the urn is started at time 0 with the vector $(N, 0,0)$ is then

$$
H\left(x_{0}, y_{0}, u_{0}, z\right)=\left(x_{0}+y_{0} z+u_{0}\left(e^{z}-1-z\right)\right)^{N} .
$$

What is of interest is the time at which a double collection is obtained, that is, all balls of $x, y$ have disappeared. Such configurations correspond to setting $\left(x_{0}, y_{0}, u_{0}\right)$ to $(0,0,1)$. Thus the probability that at time $n$ two collections have (already) been obtained is

$$
\frac{n !}{N^{n}}\left[z^{n}\right]\left(e^{z}-1-z\right)^{N}
$$

These numbers are higher order generalizations of Stirling partition numbers.

The results above are also extremely well known and in accordance with what either poissonization arguments or combinatorial enumeration techniques otherwise provide [31, 38, 40, 87]. They generalize easily to higher dimensions, thereby providing a partial unification of balls-and-bins models (random allocations [72]) and urn processes of the type studied in this paper-this aspect is otherwise discussed in the context of continuous time models by Holst [53].

The foregoing considerations are also of interest to researchers working in analysis of algorithms. Indeed, it can be verified that the algorithm known as random probing hashing (xvi) in a paging environment can be modelled by an urn of the multiple coupon collector's type. This observation has been made by Durand and Viola in 2003-2004 (unpublished) and a preliminary account of the analysis is to be found in Chapter 3 of Marianne Durand's PhD thesis [24]. (The treatment of Durand and Viola makes use of the earlier technique of characteristics, as adapted from [30].)

\subsection{The pelican's urn}

There is a tradition to be found in several cultures (Egyptian and Christian, inter alia): The mother pelican had to feed her little baby pelicans in time of famine; she would tear her breast open to feed her young with her entrails. This is rendered as follows by Alfred de Musset in his famous poem, La nuit de mai.

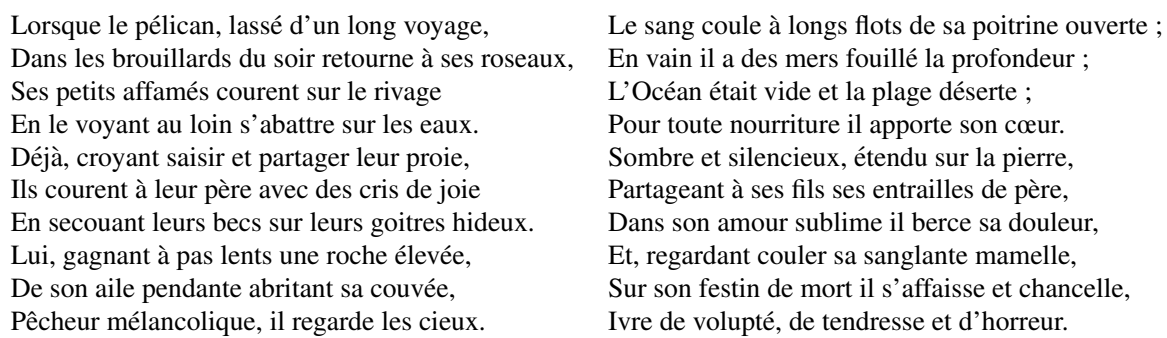

The preceding considerations perhaps justify naming as pelican's the urn whose matrix is, for $m=3$,

$$
\mathcal{M}=\left(\begin{array}{ccc}
-1 & 1 & 1 \\
1 & -1 & 1 \\
1 & 1 & -1
\end{array}\right)
$$

(xvi) See Knuth's description of the model in [71] Ex. 6.4.48]. The random probing model is a companion to the uniform probing model described in [71 pp. 534-535], and both serve to estimate the efficiency of double hashing [71] pp. 528-529]. See also the discussion in [101 pp. 507-509]. 
The associated differential system is

$$
\dot{x}=y z, \quad \dot{y}=u x, \quad \dot{u}=x y .
$$

Observe that the system is strangely reminiscent of a system of differential equation satisfied by the Jacobian elliptic functions [103, pp. 492-493]. To wit:

$$
\frac{d}{d t} \operatorname{sn} t=\operatorname{cn} t \operatorname{dn} t, \quad \frac{d}{d t} \operatorname{sn} t=-\operatorname{dn} t \operatorname{sn} t, \quad \frac{d}{d t} \operatorname{sn} t=-k^{2} \operatorname{sn} t \operatorname{cn} t,
$$

where $k$ is known as the modulus. The elliptic connection is easily elicited. In [101], multiply the first equation by $x$, the second by $y$, the third by $u$, resulting in

$$
x \dot{x}=y \dot{y}=u \dot{u}=x y u .
$$

This gives us a system of two first integrals:

$$
x^{2}-x_{0}^{2}=y^{2}-y_{0}^{2}=z^{2}-z_{0}^{2},
$$

which is sufficient to guarantee integrability. In particular, one can express $y, u$ in terms of $x$, resulting in the following differential equation for $x$ :

$$
\frac{d x}{\sqrt{\left(x^{2}-x_{0}^{2}+y_{0}^{2}\right)\left(x^{2}-x_{0}^{2}+u_{0}^{2}\right)}}=d t .
$$

This shows that indeed $x(t)$ is of the Jacobian elliptic type. The reduction to the standard normal form of Jacobian elliptic functions, namely,

$$
\frac{d w}{d t}=\sqrt{\left(1-w^{2}\right)\left(1-k^{2} w^{2}\right)}
$$

is then easily obtained upon a double change of scale:

$$
X(t)=\frac{1}{\sqrt{x_{0}^{2}-y_{0}^{2}}} \operatorname{sn}\left(\frac{t}{\sqrt{x_{0}^{2}-y_{0}^{2}}}+K, \sqrt{\frac{x_{0}^{2}-y_{0}^{2}}{x_{0}^{2}-u_{0}^{2}}}\right) .
$$

(The second argument is the explicitly written modulus; the constant $K$ is determined by initial conditions.) One can then set $x_{0}=1$ as well as $y_{0}=y, u_{0}=u$, to obtain the three fundamental functions of the pelican's urn.

It is interesting to note that the pelican's urn is related to investigations carried out by Schett in the 1970s: Schett introduced the trivariate operator

$$
\mathfrak{D}=y u \partial_{x}+u x \partial_{y}+x y \partial_{u},
$$

which is precisely the partial differential operator of the pelican's urn, and he was led to doing so for the purpose of finding efficient algorithms for calculating the Taylor expansions of the Jacobian elliptic functions [93]. Starting from there, Dumont and Viennot were then able to deduce new combinatorial interpretations of coefficients of the Jacobian elliptic functions [21, 22, 23, 100]. To this panoply, our current developments thus add histories of the pelican's urn as a new combinatorial interpretation.

The hyperpelican urn and hyperelliptic functions. Integrability is also granted for higher dimensional versions of the pelican's urn. For instance, in dimension $m=4$, the matrix

$$
\mathcal{M}=\left(\begin{array}{cccc}
-1 & 1 & 1 & 1 \\
1 & -1 & 1 & 1 \\
1 & 1 & -1 & 1 \\
1 & 1 & 1 & -1
\end{array}\right)
$$

will give rise to a hyperelliptic equation

$$
\frac{d x}{\sqrt{\left(x^{2}-a^{2}\right)\left(x^{2}-b^{2}\right)\left(x^{2}-c^{2}\right)}}=d t
$$

since (compare with 103)

$$
x \dot{x}=y \dot{y}=u \dot{u}=v \dot{v}=x y u v .
$$

We are not currently aware of works dedicated to the combinatorial interpretation of Abelian integrals (e.g., Abel's addition theorem) over curves of genus $g>1$, though some interesting combinatorics might arise. Urn models at least indicate that hyperrelliptic integrals and their inverses may well have combinatorial content. 


\section{Triangular $3 \times 3$ urns}

The theme of this section is the study of urn models with a $3 \times 3$ matrix of the triangular form

$$
\mathcal{M}=\left(\begin{array}{ccc}
\alpha & \beta & \sigma-\alpha-\beta \\
0 & \delta & \sigma-\delta \\
0 & 0 & \sigma
\end{array}\right)
$$

Such urns present a specific interest since they do not satisfy the irreducibility condition assumed in most other works. (For a notable exception, see Janson's recent study [63].)

A $3 \times 3$ triangular urn serves as yet another simple model of evolving species. There are now three species, corresponding to three types of balls, say $\mathrm{x}, \mathrm{y}, \mathrm{u}$, with respective populations $A_{n}, B_{n}, C_{n}$ at time $n$. Evolution is irreversible, following the direction $x \rightarrow y \rightarrow u$, in accordance with the following diagram:

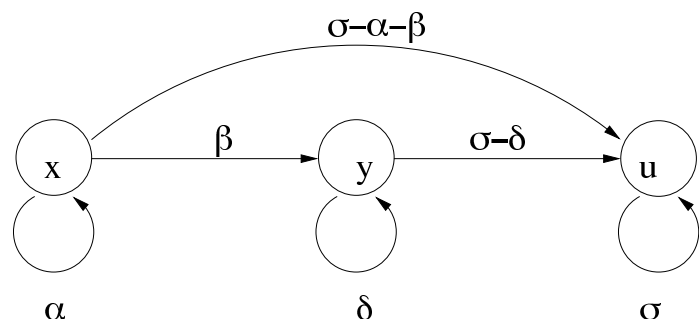

The evolution of balls of the first colour, $\mathrm{x}$, is described by a $2 \times 2$ triangular urn process, namely $\left(\begin{array}{cc}\alpha & \sigma-\alpha \\ 0 & \sigma\end{array}\right)$ (simply aggregate colours $\mathrm{y}, \mathrm{u}$ and view the corresponding balls as being of a single colour), so that we may freely restrict our attention to the random variables $B_{n}$ and $C_{n}$ representing at time $n$ the number of balls of colour $y$ and $u$, respectively. In order to avoid degenerate cases, we further assume the conditions

$$
\alpha \beta \delta \neq 0 .
$$

We now present a classification of triangular urn models with three types of balls under the assumptions (104) and (105). It turns out that three cases need to be considered: in each case, we establish convergence in distribution of $B_{n}$ and fully characterize its limit law. (Similar methods would apply to estimate $C_{n}$, while $A_{n}$ is simply described by a balanced $2 \times 2$ triangular model whose behaviour is known from $\$ 7$ ) The results of this section have been already reported in the thesis [92], to which we refer for detailed proofs.

\subsection{Generating functions}

A function $I(z)$ serves to express all generating functions relative to $3 \times 3$ urns models-it is in fact a special hypergeometric function. Its main analytic property is described by the following lemma.

Lemma 4 Let $\lambda=\frac{\alpha+\beta-\delta}{\delta}$. For any complex $z$ satisfying $|z|<1$, the function $I(z)$, defined by

$$
I(z)=\sum_{n \geq 0, n \neq \alpha / \delta}(-1)^{n} \frac{\alpha}{\alpha-n \delta}\left(\begin{array}{c}
n-\lambda-1 \\
n
\end{array}\right) z^{n}
$$

admits a unique analytic continuation to the slit complex plane $\mathbb{C} \backslash \mathbb{R}_{\leq-1}$.

Proof (sketch): The function $I(z)$ is clearly analytic in the unit disc. Define $I_{z_{0}}(z)$ by

$$
I_{z_{0}}(z)=-\alpha z^{\alpha / \delta} \int_{z_{0}}^{z^{1 / \delta}} \frac{\left(1+t^{\delta}\right)^{\lambda}}{t^{\alpha+1}} d t
$$

where $z_{0}$ is a fixed real from $\left.] 0 ; 1\right]$. By adopting as path of integration a straight line from $z_{0}$ to $z$, we see that $I_{z_{0}}$ exists as an analytic function in $\mathbb{C} \backslash \mathbb{R} \leq 0$.

Termwise integration of the expansion of the integrand of 107) provides a function which, up to a normalizing term of low degree, coincides with $I(z)$ near the origin. (In the case when $\delta$ divides $\alpha$, a further logarithmic term is to be taken into account.) 
Proposition 23 Consider a $3 \times 3$ urn of the form (104), satisfying the condition (105) and initialized with $\left(a_{0}, b_{0}, c_{0}\right)$. The 4-variable generating function of urn histories is

$$
H(x, y, u ; z)=X^{a_{0}} Y^{b_{0}} U^{c_{0}} .
$$

The functions $Y$ and $U$ are given by

$$
U=u\left(1-s u^{\sigma} z\right)^{-1 / \sigma}, \quad Y=y\left(1-y^{\delta}\left(\frac{1}{u^{\delta}}-\frac{1}{U^{\delta}}\right)\right)^{-1 / \delta} .
$$

The function $U$ is determined by cases as follows:

- If $\alpha / \delta \notin \mathbb{Z}_{\geq 1}$ :

$$
X=x\left(1-x^{\alpha}\left(I\left(\left(\frac{y}{u}\right)^{\delta}-1\right) y^{-\alpha}-I\left(\left(\frac{Y}{U}\right)^{\delta}-1\right) Y^{-\alpha}\right)\right)^{-1 / \alpha} .
$$

- If there exists $m \in \mathbb{Z}_{\geq 1}$ such that $\alpha=m \delta$, then

$$
\begin{aligned}
X=x\left(1-x^{\alpha}(I\right. & \left(\left(\frac{y}{u}\right)^{\delta}-1\right) y^{-\alpha}-I\left(\left(\frac{Y}{U}\right)^{\delta}-1\right) Y^{-\alpha} \\
& \left.\left.-\left(\begin{array}{c}
m-\lambda-1 \\
m
\end{array}\right)\left(\frac{1}{y^{\delta}}-\frac{1}{u^{\delta}}\right)^{m} \log (Y / y)^{\alpha}\right)\right)^{-1 / \alpha} .
\end{aligned}
$$

Proof (sketch): We make use of the fundamental isomorphism theorem in its multidimensional version. (A proof based instead on partial differential equations and the method of characteristics appears in [92].) The associated differential system is triangular:

$$
\left\{\begin{array} { l } 
{ \dot { x } = x ^ { \alpha + 1 } y ^ { \beta } u ^ { \sigma - \alpha - \beta } } \\
{ \dot { y } = y ^ { \delta + 1 } u ^ { \sigma - \delta } } \\
{ \dot { u } = u ^ { \sigma + 1 } }
\end{array} \quad \left\{\begin{array}{l}
x(0)=x_{0} \\
y(0)=y_{0} \\
u(0)=u_{0}
\end{array}\right.\right.
$$

The $u$-component can be solved directly,

$$
u(t)=u_{0}\left(1-\sigma u_{0}^{\sigma} t\right)^{-1 / \sigma}
$$

and the $y$-component then results by integration (since $\dot{y} y^{-\delta-1}=u^{\sigma-\delta}$ ),

$$
y(t)=y_{0}\left(1-y_{0}^{\delta}\left(u_{0}^{-\delta}-u(t)^{-\delta}\right)\right)^{-1 / \delta},
$$

just like in the two-dimensional case. The $x$-component is finally determined by another integration (since $\dot{x} x^{-\alpha-1}=y^{\beta} u^{\sigma-\alpha-\beta}$, which leads to the special function $I(z)$ defined in 106 .

\subsection{Expectation and second moment of $B_{n}$}

The probability generating function of the random variable $B_{n}$, giving the number of $\mathrm{y}$-balls at time $n$ is encoded into the GF $H(1, y, 1 ; z)$. Then, moments of any order are obtained as usual by differentiating with respect to $y$, then setting $y=1$, and finally extracting coefficients by means of the $\left[z^{n}\right]$ operation. Let $\Delta=\left(1-s z^{\sigma}\right)^{-1 / \sigma}$. The derivatives of $H$ with respect to $y$ at $y=1$ are seen to be polynomials in $\Delta$ and $\log \Delta$, whose coefficients are easily determined. Three cases emerge from the analysis.

Proposition 24 Let $B_{n}$ be the random variable giving the number of y-balls present in the urn at time $n$. Then the first and second moments of $B_{n}$ belong to one of three distinct regimes:

- If $\alpha<\delta$ :

$$
\left\{\begin{array}{l}
\mathbb{E}\left[B_{n}\right]=\left(b_{0}+a_{0} \frac{\delta}{\delta-\alpha}\right) \frac{\Gamma\left(\frac{t_{0}}{\sigma}\right)}{\Gamma\left(\frac{t_{0}+\delta}{\sigma}\right)} n^{\delta / \sigma}+O\left(n^{(\delta-1) / \sigma}\right) \\
\mathbb{E}\left[\left(B_{n}\right)^{2}\right]=\left[K \frac{\Gamma\left(\frac{t_{0}}{\sigma}\right)}{\Gamma\left(\frac{t_{0}+2 \alpha}{\sigma}\right)}\right] n^{2 \delta / \sigma}+O\left(n^{(2 \delta-1) / \sigma}\right)
\end{array}\right.
$$

where $K$ is

$$
K=\left(b_{0}+a_{0} \frac{\delta}{\delta-\alpha}\right)^{2}+\delta b_{0}+a_{0}\left(\frac{\alpha \delta^{2}}{(\delta-\alpha)^{2}}+\delta-\alpha-2 \delta+\frac{2 \delta(\delta+\delta)}{2 \delta-\alpha}\right) .
$$


- If $\alpha=\delta:$

$$
\left\{\begin{array}{l}
\mathbb{E}\left[B_{n}\right]=\frac{a_{0} \delta}{\alpha} \frac{\Gamma\left(\frac{t_{0}}{\sigma}\right)}{\Gamma\left(\frac{t_{0}+\alpha}{\sigma}\right)} n^{\alpha / \sigma} \log n+O\left(n^{\alpha / \sigma}\right) \\
\mathbb{E}\left[\left(B_{n}\right)^{2}\right] \sim\left(\frac{a_{0} \delta}{\alpha}\right)^{2}\left[\left(1+\frac{\alpha}{a_{0}}\right) \frac{\Gamma\left(\frac{t_{0}}{\sigma}\right)}{\Gamma\left(\frac{t_{0}+2 \alpha}{\sigma}\right)}\right] n^{2 \alpha / \sigma}(\log n)^{2}+O\left(n^{2 \alpha / \sigma} \log n\right) .
\end{array}\right.
$$

- If $\alpha>\delta$ :

$$
\left\{\begin{array}{l}
\mathbb{E}\left[B_{n}\right]=\frac{a_{0} \delta}{\alpha-\delta} \frac{\Gamma\left(\frac{t_{0}}{\sigma}\right)}{\Gamma\left(\frac{t_{0}+\alpha}{\sigma}\right)} n^{\alpha / \sigma}+O\left(n^{(\alpha-1) / \sigma}\right) \\
\mathbb{E}\left[\left(B_{n}\right)^{2}\right]=\left(\frac{a_{0} \delta}{\alpha-\delta}\right)^{2}\left[\left(1+\frac{\alpha}{a_{0}}\right) \frac{\Gamma\left(\frac{t_{0}}{\sigma}\right)}{\Gamma\left(\frac{t_{0}+2 \alpha}{\sigma}\right)}\right] n^{2 \alpha / \sigma}+O\left(n^{2(\alpha-1) / \sigma}\right) .
\end{array}\right.
$$

This result, when accompanied by similar analyses of $C_{n}$ (the number of u-balls) and $A_{n}$ (the number of x-balls), provides an overall picture of the urn's probable evolutions. First, since $A_{n}$ is governed by a $2 \times 2$ triangular urn process, we have with high probability [w.h.p.]: $A_{n}=\Theta\left(n^{\alpha / \sigma}\right)$, given what we already know regarding $2 \times 2$ triangular urns ( $\$ 7$, p. 93. Naturally, we have $A_{n}+B_{n}+C_{n}=\sigma n+O(1)$, which implies $B_{n}+C_{n}=\sigma n+o(n)$ [w.h.p]; next $B_{n}=o(n)$ holds according to Proposition 24, so that $C_{n}=\sigma n+o(n)$ [w.h.p.]. We may then regard intuitively the diagonal quantities as reproduction rates and interpret the situation as follows: $(i)$ if $\alpha<\delta$, the reproduction rate of $\mathrm{x}$-balls is smaller than that of y-balls, so that y balls evolve essentially at their own pace, which corresponds to $B_{n}$ being of order $n^{\delta / \sigma}$. (ii) if $\alpha=\delta$, the reproduction rates of $\mathrm{x}$ - and $\mathrm{y}$-balls are the same, and from this confluence of values, a logarithmic term is induced; (iii) if $\alpha>\delta$, then $\mathrm{x}$-balls, which reproduce faster, drive the game for $\mathrm{y}$-balls.

\subsection{The distribution of $B_{n}$ when $\alpha \geq \delta$}

This is the case where the evolution of y-balls is essentially driven by that of $x$-balls. We treat it first as it is technically simpler. We have:

Proposition 25 For a triangular urn with $\alpha \geq \delta$, the random variable $B_{n}$ giving the number of $\mathrm{y}$-balls at time $n$ admits moments that satisfy the following asymptotic estimates:

$$
- \text { If } \alpha>\delta \text { : }
$$$$
E\left[B_{n}^{\ell}\right]=\left(\frac{\alpha \delta}{\alpha-\delta}\right)^{\ell} \frac{\Gamma\left(\frac{a_{0}+\ell \alpha}{\alpha}\right) \Gamma\left(\frac{t_{0}}{\sigma}\right)}{\Gamma\left(\frac{a_{0}}{\alpha}\right) \Gamma\left(\frac{t_{0}+\ell \alpha}{\sigma}\right)} n^{\ell \alpha / \sigma}+O\left(n^{\ell(\alpha-1) / \sigma}\right)
$$

- If $\alpha=\delta$, then

$$
E\left[B_{n}^{\ell}\right]=\delta^{\ell} \frac{\Gamma\left(\frac{a_{0}+\ell \alpha}{\alpha}\right) \Gamma\left(\frac{t_{0}}{\sigma}\right)}{\Gamma\left(\frac{a_{0}}{\alpha}\right) \Gamma\left(\frac{t_{0}+\ell \alpha}{\sigma}\right)} n^{\ell a / \sigma}(\log n)^{\ell}+O\left(n^{\ell \alpha / \sigma}(\log n)^{\ell-1}\right) .
$$

In particular, the normalized variable $\bar{B}_{n}$ defined by

$$
\bar{B}_{n}=\frac{\alpha}{\delta} \frac{B_{n}}{n^{\alpha / \delta} \log n} \quad \text { if } \quad \alpha=\delta, \quad \bar{B}_{n}=\frac{\alpha-\delta}{\delta} \frac{B_{n}}{n^{\alpha / \delta}} \quad \text { otherwise. }
$$

converges in distribution to a limit low whose density is given by 807 ,

Proof (sketch): The moment calculations are detailed in [92]. A comparison with the moments obtained for the $2 \times 2$ case shows that they are the same to main asymptotic order, up to scaling. The convergence in distribution then follows from the classical moment convergence theorem.

\subsection{The distribution of $B_{n}$ when $\alpha<\delta$}

In this case, the function $H(1, y, 1 ; z)$ admits a series expansion at $y=1$ whose coefficients are polynomials in $\Delta$ and $\log \Delta$; the coefficient of $(y-1)^{\ell}$ has degree $\ell \delta$ in $\Delta$ and its leading term involves a rational function of $a_{0}, b_{0}, \alpha, \beta, \delta$. Hence, each moment of the normalized random variable $\bar{B}_{n}=B_{n} / n^{\delta / \sigma}$ converges to a finite value. The first few terms are easily obtained by series expansions (and computer algebra!), but we have been unable to find a simple expression for the general term. The following proposition establishes a convergence in distribution for this random variable as well as an integral representation of the characteristic function. (This representation can then be used to calculate moments at will, via successive differentiations.) 
Proposition 26 For a triangular urn with $\alpha<\delta$, the normalized random variable

$$
\bar{B}_{n}=\frac{B_{n}}{n^{\delta / \sigma}}
$$

converges in distribution to the law whose characteristic function admits the integral representation

$$
\phi(\xi)=\frac{1}{2 i \pi} \Gamma\left(\frac{t_{0}}{\sigma}\right) \int_{\mathcal{H}} t^{-c_{0} / \sigma}\left(t^{\rho}-i \delta \xi\right)^{-\left(a_{0}+b_{0}\right) / \delta} I\left(\frac{i \delta \xi}{t^{\rho}-i \delta \xi}\right)^{-a_{0} / \alpha} e^{t} d t .
$$

For any $\xi$ in $\left[-\xi_{0} ; \xi_{0}\right]$, the contour $\mathcal{H}$ can be any clockwise oriented contour surrounding the negative real axis at a distance at least $\left(1+\frac{1}{R}\right)^{1 / \rho}\left(\delta \xi_{0}\right)^{1 / \rho}$, where $R$ is such that $I^{-1 / \alpha}$ is well defined and bounded for $|z| \leq R$.

Proof (sketch): We only treat the case of an urn initialized with one $\mathrm{x}$-ball at time 0 , the general case being similar. The idea consists in extracting the coefficient of $z^{n}$ in $H(1, y, 1 ; z)$ by means of Cauchy's coefficient formula: this gives the probability generating function of $C_{n}$, up to a factor of $H_{n}$. For this coefficient extraction, a Hankel contour in accordance with the principles of singularity analysis is adopted. At the same time, since convergence in law depends on a suitably scaled version of $B_{n}$, the substitution

$$
y=e^{i \xi / n^{\delta / \sigma}}
$$

is to be used. This renormalization, combined with standard asymptotic approximations, then gives rise to the integral representation (111).

Precisely, set $\rho=\delta / \sigma$. The characteristic function $\phi_{n}(t)$ of $\bar{B}_{n}$ is

$$
\phi_{n}(\xi) \equiv \mathbb{E}\left[e^{i \xi \bar{B}_{n}}\right]=\frac{n !}{1 \cdot(1+\sigma) \cdots(1+(n-1) \sigma)}\left[z^{n}\right] H\left(1, e^{i \xi / n^{\rho}}, 1 ; z\right) .
$$

By the continuity theorem of characteristic functions, it suffices to show that the function $\phi_{n}$ converges to $\phi$, as given by (111). Let $\xi$ be a fixed real number taken from an interval $\left[-\xi_{0}, \xi_{0}\right]$. The coefficient of $\left[z^{n}\right]$ is obtained by means of Cauchy's coefficient formula, which gives (after the change of variable $\sigma z \rightarrow z$ )

$$
\phi_{n}(\xi)=\frac{n !}{\frac{1}{\sigma} \cdots\left(\frac{1}{\sigma}+n-1\right)} \frac{1}{2 i \pi} \oint h\left(z, \frac{\xi}{n^{\beta}}\right)^{-\frac{1}{\alpha}} \frac{d z}{z^{n+1}},
$$

where the function $h$ is

$$
h(z, \theta)=1-\frac{I\left(e^{i \delta \theta}-1\right)}{e^{a \theta}}+I\left(\frac{(1-z)^{-\rho}\left(1-e^{-i \delta \theta}\right)}{1-(1-z)^{-\rho}\left(1-e^{-i \delta \theta}\right)}\right)\left(e^{-i \delta \theta}-1+(1-z)^{\rho}\right)^{\alpha / \delta} .
$$

As already announced, we choose the integration contour to be of the Hankel type, as in Figure 9 Then we renormalize $z$ by setting $z=1-t / n$, in accordance with the general principles of singularity analysis. One then finds the main approximation:

$$
h\left(1-\frac{t}{n}, \frac{\xi}{n^{\rho}}\right)=I\left(\frac{i \delta \xi+O\left(\frac{1}{n^{\rho}}\right)}{t^{\rho}-i \delta \xi+O\left(\frac{1}{n^{\rho}}\right)}\right)\left(t^{\rho}-i \delta \xi+O\left(\frac{1}{n^{\rho}}\right)\right)^{\alpha / \delta}+O\left(\frac{1}{n^{(\delta-\alpha) / \sigma}}\right) .
$$

Integration of this last approximation over a complete Hankel contour only introduces negligible error terms (see [92] for details), a fact which concludes the proof of the statement.

The results above have some striking formal similarities with the analysis given by Janson of unbalanced $2 \times 2$ urn models. Perhaps this could be explained to some extent by considering that the $x$ - and $y$-balls play between themselves a game that resembles the one described by an unbalanced $2 \times 2$ model.

\section{Relations between discrete and continuous time models}

Since a large portion of the literature dedicated to urns appeals to a continuous time model, it is of obvious interest to investigate whether a dictionary exists between the continuous and discrete frameworks. Elements for this discussion can be found in the book by Athreya and Ney [4] (see p. 219-224 for a specific 
treatment of the relationships between urns and branching processes), the article of Athreya and Karlin [3], as well as in the dedicated calculation of [16, §3.4].

The continuous time model assumes that each ball is equipped with an exponentially distributed, $\operatorname{Exp}(1)$, clock. In other words, the random time $T$ at which a ball fires a transition (after it has been born) satisfies

$$
\mathbb{P}(T>t)=e^{-t}
$$

The exponential distribution satisfies the well-known memoryless property, namely

$$
\mathbb{P}(T>t+\tau \mid T>\tau)=\mathbb{P}(T>t),
$$

which entails a fundamental equivalence principle (see [4, p. 221] and [3, Th. 1]): The discrete time urn process at any instant $n=0,1,2, \ldots$ and the continuous time version stopped after the nth firing are equivalent.

Consider a balanced urn process given as usual by a matrix $\mathcal{M}$ with balance $\sigma$. Let $\mathbf{Z}(t)$ represent the continuous time urn process at time $t$, and let $\mathbf{Y}_{n}$ be the discrete urn process at time $n$ (our notations follow [4]). We can now state a quantitative version of the equivalence principle.

Theorem 7 (Discrete-to-continuous transfer) Consider a balanced urn model with balance $\sigma$. Let $\mathcal{E}$ be an event that is defined by a set of histories ${ }^{(x v i i)}$ also denoted by $\mathcal{E}$. Define the continuous time probabilities:

$$
\Omega(t):=\mathbb{P}(\mathbf{Z}(t) \in \mathcal{E})
$$

and the history counts

$$
\Xi_{n}:=\operatorname{card}\{h \in \mathcal{E}|| h \mid=n\} .
$$

Let the urn be initialized with $s_{0}$ balls at time 0. Then, the continuous time probabilities are given by

$$
\Omega(t)=e^{-s_{0} t} \Xi\left(\frac{1}{\sigma}\left(1-e^{-\sigma t}\right)\right),
$$

where $\Xi(z)$ is the exponential generating function of the sequence of history counts $\left(\Xi_{n}\right)$ :

$$
\Xi(z):=\sum_{n \geq 0} \Xi_{n} \frac{z^{n}}{n !} .
$$

Proof: The proof boils down to the determination of the distribution of the urn's size in continuous time, the principles having been folklore knowledge for a long time (see [4, p. 109] for the generalized Yule process, [63, Lemma 5.1], and [16, §3.4] for a calculation analogous to the present one). We have, upon conditioning on size,

$$
\mathbb{P}(\mathbf{Z}(t) \in \mathcal{E})=\sum_{n \geq 0} \mathbb{P}(S(t)=n) \frac{\Xi_{n}}{H_{n}},
$$

where $S(t)$ is the random variable representing the size of the continuous time urn at time $t$.

Regarding the distribution of $S(t)$, we have

$$
\mathbb{P}[S(t+d t)=n+\sigma]=\mathbb{P}[S(t)=n+\sigma] \cdot(1-(n+\sigma) d t)+\mathbb{P}[S(t)=n] \cdot n d t .
$$

(Either size has not changed as none of the $(n+\sigma)$ balls fired in the interval $[t, t+d t]$, or size was previously $n$ and one of the $n$ balls fired. This is an instance of the Kolmogorov backward equations.) Set $\varpi_{n}(t)=\mathbb{P}(S(t)=n)$. Then, Equation 115 can be rephrased as the differential recurrence

$$
\varpi_{n+\sigma}^{\prime}(t)+(n+\sigma) \varpi_{n+\sigma}(t)=n \varpi_{n}(t)
$$

The differential equation satisfied by $\varpi_{n+\sigma}$ is then solved by the variation-of-constant method, which provides an integral recurrence:

$$
\varpi_{n+\sigma}(t)=n e^{-(n+\sigma) t} \int_{0}^{t} e^{(n+\sigma) t} \varpi_{n}(t) d t,
$$

(xvii) Acceptable events are for instance: $(i)$ the urn contains $k$ balls of the first type; $(i i)$ the urn contains $k$ balls of the first type at time $n$; (iii) the urn has more balls of the first type than of the second type at all even instants; $(i v)$ a ball of the second type has never been drawn; $(v)$ all balls of the second type have disappeared in the end; and so on. 
whose solution is easily verified to be

$$
\varpi_{n}(t) \equiv \mathbb{P}(S(t)=n)=e^{-s_{0} t}\left(1-e^{-\sigma t}\right)^{n}\left(\begin{array}{c}
n+s_{0} / \sigma-1 \\
n
\end{array}\right) .
$$

It then suffices to observe that $H_{n}$, as expressed by Proposition 1 (p. 63, equals $n ! \sigma^{n}\left(\begin{array}{c}n+s_{0} / \sigma-1 \\ n\end{array}\right)$, and the proof is completed by combining (113) and the evaluation (117).

We can first conduct a few verification. Let $\mathcal{E}=\{\epsilon\}$, i.e., only the "empty" history of size 0 is in $\mathcal{E}$. We have $\Xi(z)=1$ and Theorem 7 predicts $\Omega(t)=e^{-s_{0} t}$, which is consistent with the fact that no firing amongst the $s_{0}$ original balls should have taken place till time $t$. Next, let $\mathcal{E}$ be the set of all histories: the generating function is, by Proposition $1 . \Xi(z) \equiv H(z)=[1-\sigma z]^{-s_{0} / \sigma}$. Substitution then shows that

$$
\Omega(t)=e^{-s_{0} t}\left[1-\left(1-e^{-\sigma t}\right)\right]^{-s_{0} / \sigma} \equiv 1,
$$

as it should be. Naturally, the statement of Theorem 7 can be extended by linearity to generating functions. For instance, we have:

Proposition 27 Let $(A(t), B(t))$ be the composition of a balanced $2 \times 2$ urn at time $t$ and let, as usual, $H(x, y, z)$ be the trivariate generating function of urn histories. The continuous and discrete models are related by

$$
\sum_{a, b} \mathbb{P}[(A(t), B(t))=(a, b)] x^{a} y^{b}=e^{-s_{0} t} H\left(x, y, \frac{1}{\sigma}\left(1-e^{-\sigma t}\right)\right) .
$$

As a consequence of Theorem 7 and 27. essentially all our previous calculations relative to balanced urns in discrete time can be translated back into the framework of continuous time. Take for instance the generation-parity model of Example 4, p. 79, initialized with one x-ball. Under continuous time, the probability that, at some large time $t$, all balls be of an odd generation - this is an "extreme" large deviation event-is

$$
e^{-t} \operatorname{smh}\left(1-e^{-t}\right)
$$

$\left(\operatorname{smh}(z)\right.$ is defined in (43)), and is thus exponentially small, being asymptotic to $e^{-t} \operatorname{smh}(1)$, where $\operatorname{smh}(1) \doteq 1.20541$.

\section{Conclusions}

A first conclusion of this preliminary study is that some order can be put amongst those classical urn models that admit of explicit solutions. More generally, the methods of analytic combinatorics, based on generating functions, complex asymptotics and singularities, as well as the Quasi-Powers Theorem (for Gaussian laws) and specific contour integration (for non-Gaussian laws) can provide a wealth of valuable information. In this way, we have been able to obtain precise estimates of speed of convergence, some local limit convergence results, large deviation rate functions, and, in a few cases even, limit laws that seem to be new.

Many more problems deserve further investigation. We list them here in order of increasing difficulty.

- The study of nonsacrificial urns (either altruistic or selfish in the sense of $\$ 3$ p. 74 should be conducted in a way that parallels our study of semi-sacrificial urns. We foresee no particular obstacle in the case where the urn's composition is Gaussian, as the Quasi-Powers Theorem should be applicable. Non-Gaussian cases should be amenable to treatment by methods of contour integration perhaps analogous to the ones employed for triangular models (see [38, Ch. IX] for a discussion of some relevant methods).

- The study of higher dimensional models might be amenable to a perturbative analysis of singularities, at least in the case where the urn has a Gaussian behaviour, and despite the fact that there is no hope to obtain general solutions because of nonintegrability considerations (Note 11, p. 102).

- The elucidation of the behaviour of at least some of the non-balanced models seems to be the most challenging problem posed to the analytic approach at this stage.

Acknowledgements. The authors are grateful to Philippe Chassaing, Yoshiaki Itoh, Nicolas Pouyanne, Philippe Robert, Aleksandar Sedoglavić, Brigitte Vallée, and Jacques-Arthur Weil for many stimulating discussions and valuable bibliographical indications. 


\section{References}

[1] David Aldous, Asymptotic fringe distributions for general families of random trees, Annals of Applied Probability 1 (1991), 228-266.

[2] David Aldous, Barry Flannery, and José Luis Palacios, Two applications of urn processes-the fringe analysis of trees and the simulation of quasi-stationary distributions of Markov chains, Probability in the Engineering and Informational Sciences 2 (1988), 293-307.

[3] Krishna B. Athreya and Samuel Karlin, Embedding of urn schemes into continuous time Markov branching processes and related limit theorems, Ann. Math. Statist. 39 (1968), 1801-1817. MR MR0232455 (38 \#780)

[4] Krishna B. Athreya and Peter E. Ney, Branching processes, Springer-Verlag, New York, 1972, Die Grundlehren der mathematischen Wissenschaften, Band 196.

[5] Ricardo A. Baeza-Yates, Fringe analysis revisited, ACM Computing Surveys 27 (1995), no. 1, 109-119.

[6] A. Bagchi and A. K. Pal, Asymptotic normality in the generalized Pólya-Eggenberger urn model, with an application to computer data structures, SIAM Journal on Algebraic and Discrete Methods 6 (1985), no. 3, 394-405.

[7] Cyril Banderier, Philippe Flajolet, Gilles Schaeffer, and Michèle Soria, Random maps, coalescing saddles, singularity analysis, and Airy phenomena, Random Structures \& Algorithms 19 (2001), no. 3/4, 194-246.

[8] Rodney J. Baxter, Exactly solved models in statistical mechanics, Academic Press Inc. [Harcourt Brace Jovanovich Publishers], London, 1982.

[9] F. Bergeron, G. Labelle, and P. Leroux, Combinatorial species and tree-like structures, Cambridge University Press, Cambridge, 1998.

[10] François Bergeron, Philippe Flajolet, and Bruno Salvy, Varieties of increasing trees, CAAP'92 (J.-C. Raoult, ed.), Lecture Notes in Computer Science, vol. 581, 1992, Proceedings of the 17th Colloquium on Trees in Algebra and Programming, Rennes, France, February 1992., pp. 24-48.

[11] Norman L. Biggs, Algebraic graph theory, Cambridge University Press, 1974.

[12] William H. Burge, An analysis of a tree sorting method and some properties of a set of trees, First USA-JAPAN Computer Conference Proceedings, AFIPS and IPSJ, October 1972, pp. 372-378.

[13] K. Chandrasekharan, Elliptic functions, Grundlehren der Mathematischen Wissenschaften [Fundamental Principles of Mathematical Sciences], vol. 281, Springer-Verlag, Berlin, 1985.

[14] Laurent Cheno, Philippe Flajolet, Jean Françon, Claude Puech, and Jean Vuillemin, Dynamic data structures: Finite files, limiting profiles and variance analysis, Eighteenth Annual Conference on Communication, Control, and Computing, The University of Illinois at Urbana-Champaign, 1980, pp. 223-232.

[15] Louis Comtet, Advanced combinatorics, Reidel, Dordrecht, 1974.

[16] Eric van Fossen Conrad and Philippe Flajolet, The Fermat cubic, elliptic functions, continued fractions, and a combinatorial excursion, Séminaire Lotharingien de Combinatoire 54 (2006), no. B54g, 1-44.

[17] Benoît Daireaux, Véronique Maume-Deschamps, and Brigitte Vallée, The Lyapunov tortoise and the dyadic hare, 2005 International Conference on Analysis of Algorithms, Discrete Mathematics \& Theoretical Computer Science Proceedings, vol. AD, 2005, pp. 71-94 (electronic).

[18] F. N. David and D. E. Barton, Combinatorial chance, Charles Griffin, London, 1962.

[19] Frank den Hollander, Large deviations, American Mathematical Society, Providence, RI, 2000.

[20] Alfred Cardew Dixon, On the doubly periodic functions arising out of the curve $x^{3}+y^{3}-3 \alpha x y=1$, The Quarterly Journal of Pure and Applied Mathematics 24 (1890), 167-233.

[21] Dominique Dumont, A combinatorial interpretation for the Schett recurrence on the Jacobian elliptic functions, Mathematics of Computation 33 (1979), 1293-1297.

[22] _ Une approche combinatoire des fonctions elliptiques de Jacobi, Advances in Mathematics 1 (1981), $1-39$.

[23] _ Grammaires de William Chen et dérivations dans les arbres et arborescences, Séminaire Lotharingien de Combinatoire 37 (1996), Art. B37a, 21 pp. (electronic).

[24] Marianne Durand, Combinatoire analytique et algorithmique des ensembles de données, Ph.D. thesis, École Polytechnique, France, 2004.

[25] A. Edelman and E. Kostlan, The road from Kac's matrix to Kac's random polynomials, Proceedings of the 1994 SIAM Applied Linear Algebra Conference (Philadelphia) (J.G. Lewis, ed.), SIAM, 1994, pp. 503-507.

[26] Paul Ehrenfest and Tatiana Ehrenfest, Über zwei bekannte Einwände gegen das Boltzmannsche H-Theorem, Physikalische Zeitschrift 8 (1907), no. 9, 311-314.

[27] W. Feller, An introduction to probability theory and its applications, vol. 2, John Wiley, 1971. 
[28] Philippe Flajolet, Combinatorial aspects of continued fractions, Discrete Mathematics 32 (1980), 125-161, Reprinted in the 35th Special Anniversary Issue of Discrete Mathematics, Volume 306, Issue 10-11, Pages 992-1021 (2006).

[29] Philippe Flajolet and J. Françon, Structures de données dynamiques en reservoir borné, III Journées Algorithmiques (J. Morgenstern, ed.), Université de Nice, 1980, 14 pages. (Proceedings of a meeting, June 1980).

[30] Philippe Flajolet, Joaquim Gabarró, and Helmut Pekari, Analytic urns, Annals of Probability 33 (2005), no. 3, 1200-1233, Available from ArXiv: math.PR/0407098.

[31] Philippe Flajolet, Danièle Gardy, and Loÿs Thimonier, Birthday paradox, coupon collectors, caching algorithms, and self-organizing search, Discrete Applied Mathematics 39 (1992), 207-229.

[32] Philippe Flajolet, Xavier Gourdon, and Conrado Martínez, Patterns in random binary search trees, Random Structures \& Algorithms 11 (1997), no. 3, 223-244.

[33] Philippe Flajolet and Fabrice Guillemin, The formal theory of birth-and-death processes, lattice path combinatorics, and continued fractions, Advances in Applied Probability 32 (2000), 750-778.

[34] Philippe Flajolet and Andrew M. Odlyzko, Singularity analysis of generating functions, SIAM Journal on Algebraic and Discrete Methods 3 (1990), no. 2, 216-240.

[35] Philippe Flajolet, Patricio Poblete, and Alfredo Viola, On the analysis of linear probing hashing, Algorithmica 22 (1998), no. 4, 490-515.

[36] Philippe Flajolet and Vincent Puyhaubert, Analytic combinatorics at OK Corral, Technical memorandum, 2005, To be submitted.

[37] Philippe Flajolet and Robert Sedgewick, Mellin transforms and asymptotics: finite differences and Rice's integrals, Theoretical Computer Science 144 (1995), no. 1-2, 101-124.

[38] __ Analytic combinatorics, April 2006, Chapters I-IX of a book to be published by Cambridge University Press, 717p.+x, available electronically from P. Flajolet's home page.

[39] Philippe Flajolet and Michèle Soria, General combinatorial schemas: Gaussian limit distributions and exponential tails, Discrete Mathematics 114 (1993), 159-180.

[40] Dominique Foata, Bodo Lass, and Guo-Niu Han, Les nombres hyperharmoniques et la fratrie du collectionneur de vignettes, Seminaire Lotharingien de Combinatoire 47 (2001), no. B47a, 1-20.

[41] J. Françon, G. Viennot, and Jean Vuillemin, Description and analysis of an efficient priority queue representation, Proceedings of the 19th Annual Symposium on Foundations of Computer Science, IEEE Computer Press, 1978, pp. 1-7.

[42] Jean Françon and Gérard Viennot, Permutations selon leurs pics, creux, doubles montées et doubles descentes, nombres d'Euler et de Genocchi, Discrete Mathematics 28 (1979), 21-35.

[43] Bernard Friedman, A simple urn model, Communications in Pure and Applied Mathematics 2 (1949), 59-70.

[44] J. Gani, Random-allocation and urn models, Journal of Applied Probability 41A (2004), 313-320, Stochastic methods and their applications.

[45] Alexander Gnedin and Jim Pitman, Exchangeable Gibbs partitions and Stirling triangles, Preprint available on ArXiv, 2004, arXiv.org:math/0412494.

[46] Raúl Gouet, A martingale approach to strong convergence in a generalized Pólya-Eggenberger urn model, Statistics \& Probability Letters 8 (1989), no. 3, 225-228. MR MR1024032 (91d:60075)

[47] _ Martingale functional central limit theorems for a generalized Pólya urn, The Annals of Probability 21 (1993), no. 3, 1624-1639.

[48] __ Strong convergence of proportions in a multicolor Pólya urn, Journal of Applied Probability 34 (1997), no. 2, 426-435.

[49] Ian P. Goulden and David M. Jackson, Combinatorial enumeration, John Wiley, New York, 1983.

[50] _ Distributions, continued fractions, and the Ehrenfest urn model, Journal of Combinatorial Theory. Series A 41 (1986), no. 1, 21-31.

[51] Ronald L. Graham, Donald E. Knuth, and Oren Patashnik, Concrete mathematics, Addison Wesley, 1989.

[52] I. Higueras, J. Moler, F. Plo, and M. San Miguel, Urn models and differential algebraic equations, Journal of Applied Probability 40 (2003), no. 2, 401-412.

[53] Lars Holst, A unified approach to limit theorems for urn processes, Journal of Applied Probability 16 (1979), $154-162$.

[54] Fred M. Hoppe, Pólya-like urns and the Ewens sampling formula, J. Math. Biology 20 (1984), 91-94.

[55] Hsien-Kuei Hwang, Théorèmes limites pour les structures combinatoires et les fonctions arithmetiques, Ph.D. thesis, École Polytechnique, December 1994. 
[56] Large deviations for combinatorial distributions. I. Central limit theorems, The Annals of Applied Probability 6 (1996), no. 1, 297-319.

[57]_ Large deviations of combinatorial distributions. II. Local limit theorems, The Annals of Applied Probability 8 (1998), no. 1, 163-181.

[58] On convergence rates in the central limit theorems for combinatorial structures, European Journal of Combinatorics 19 (1998), no. 3, 329-343.

[59] Yoshiaki Itoh, On a ruin problem with interaction, Annals of the Institute of Statistical Mathematics 25 (1973), 635-641. MR MR0346951 (49 \#11671)

[60] _ Random collision models in oriented graphs, Journal of Applied Probability 16 (1979), no. 1, 36-44.

[61] Yoshiaki Itoh, Colin Mallows, and Larry Shepp, Explicit sufficient invariants for an interacting particle system, Journal of Applied Probability 35 (1998), no. 3, 633-641.

[62] Svante Janson, Functional limit theorems for multitype branching processes and generalized Pólya urns, Stochastic Processes and Applications 110 (2004), no. 2, 177-245.

[63] __ Limit theorems for triangular urn schemes, Probability Theory and Related Fields 134 (2006), 417-452.

[64] Norman L. Johnson and Samuel Kotz, Urn models and their application, John Wiley, 1977.

[65] J. P. Jouanolou, Équations de Pfaff algébriques, Lecture Notes in Mathematics, vol. 708, Springer, Berlin, 1979.

[66] Mark Kac, Random walk and the theory of Brownian motion, American Mathematical Monthly 54 (1947), 369391.

[67] Samuel Karlin and James McGregor, Ehrenfest urn models, Journal of Applied Probability 2 (1965), 352-376.

[68] J. F. C. Kingman, Martingales in the OK Corral, The Bulletin of the London Mathematical Society 31 (1999), no. 5, 601-606.

[69] __ Stochastic aspects of Lanchester's theory of warfare, Journal of Applied Probability 39 (2002), no. 3, 455-465.

[70] J. F. C. Kingman and S. E. Volkov, Solution to the OK Corral model via decoupling of Friedman's urn, Journal of Theoretical Probability 16 (2003), no. 1, 267-276.

[71] Donald E. Knuth, The art of computer programming, 2nd ed., vol. 3: Sorting and Searching, Addison-Wesley, 1998.

[72] Valentin F. Kolchin, Boris A. Sevastyanov, and Vladimir P. Chistyakov, Random allocations, John Wiley and Sons, New York, 1978, Translated from the Russian original Slučajnye Razmeščeniya.

[73] Samuel Kotz and N. Balakrishnan, Advances in urn models during the past two decades, Advances in combinatorial methods and applications to probability and statistics, Stat. Ind. Technol., Birkhäuser Boston, Boston, MA, 1997, pp. 203-257. MR MR1456736 (98f:60012)

[74] Samuel Kotz, Hosam Mahmoud, and Philippe Robert, On generalized Pólya urn models, Statistics \& Probability Letters 49 (2000), no. 2, 163-173.

[75] Pierre-Simon Laplace, Théorie analytique des probabilités. Vol. I, II, Éditions Jacques Gabay, Paris, 1995, Reprint of the 1819 and 1820 editions.

[76] P. Leroux and G. X. Viennot, Combinatorial resolution of systems of differential equations I: Ordinary differential equations, Combinatoire Énumérative (G. Labelle and P. Leroux, eds.), Lecture Notes in Mathematics, no. 1234, Springer-Verlag, 1986, pp. 210-245.

[77] _ A Combinatorial Approach to Non Linear Functional Expansions: An Introduction With an Example, Proceedings of the 27th IEEE Conference on Decision and Control (Austin, Texas), December 1988, pp. 13141319.

[78] Peter Lindqvist, Some remarkable sine and cosine functions, Ricerche di Matematica XLIV (1995), no. 2, 269290.

[79] Peter Lindqvist and Jaak Peetre, Two remarkable identities, called twos, for inverses to some Abelian integrals, The American Mathematical Monthly 108 (2001), no. 5, 403-410.

[80] Eugene Lukacs, Characteristic functions, Griffin, London, 1970.

[81] Erik Lundberg, Om hypergoniometriskafunktioner af komplexa variabla, Manuscript, 1879, English translation, "On hypergoniometric functions of complex variables" available from Jaak Peetre's web page.

[82] Andrzej J. Maciejewski, Jean Moulin Ollagnier, Andrzej Nowicki, and Jean-Marie Strelcyn, Around Jouanolou non-integrability theorem, Indagationes Mathematicae, New Series 11 (2000), no. 2, 239-254.

[83] Hosam Mahmoud, On rotations in fringe-balanced binary trees, Information Processing Letters 65 (1998), 4146. 
[84] U Urn models and connections to random trees: a review, Journal of the Iranian Mathematical Society 2 (2003), 53-114.

[85] Bojan Mohar, Some applications of Laplace eigenvalues of graphs, Graph Symmetry: Algebraic Methods and Applications (G. Hahn and G. Sabidussi, eds.), NATO ASI Ser., vol. C 497, Kluwer, 1997, pp. 225-275.

[86] Jean Moulin Ollagnier, Andrzej Nowicki, and Jean-Marie Strelcyn, On the non-existence of constants of derivations: the proof of a theorem of Jouanolou and its development, Bulletin des Sciences Mathématiques 119 (1995), no. 3, 195-233.

[87] Donald J. Newman and Lawrence Shepp, The double dixie cup problem, American Mathematical Monthly 67 (1960), 58-61.

[88] Alois Panholzer and Helmut Prodinger, An analytic approach for the analysis of rotations in fringe-balanced binary search trees, Annals of Combinatorics 2 (1998), 173-184.

[89] Jim Pitman, Combinatorial stochastic processes, Technical Report 621, 2002, Lecture Notes for Saint-Flour Course, 231 pages.

[90] Nicolas Pouyanne, An algebraic approach of large Pólya processes, To appear in Annales de l'Institut Henri Poincaré, 40 pages, in press.

[91] _ Classification of large Pólya-Eggenberger urns with regard to their asymptotics, 2005 International Conference on Analysis of Algorithms, Discrete Mathematics and Theoretical Computer Science (Proceedings), vol. AD, 2005, pp. 275-285 (electronic).

[92] Vincent Puyhaubert, Modèles d'urnes et phénomènes de seuil en combinatoire analytique, PhD thesis, École Polytechnique, Palaiseau, France, 2005.

[93] Alois Schett, Properties of the Taylor series expansion coefficients of the Jacobian elliptic functions, Mathematics of Computation 30 (1976), no. 133, 143-147.

[94] Robert Sedgewick and Philippe Flajolet, An introduction to the analysis of algorithms, Addison-Wesley Publishing Company, 1996.

[95] N. J. A. Sloane, The On-Line Encyclopedia of Integer Sequences, 2006, Published electronically at www.research.att.com/ njas/sequences/.

[96] R. T. Smythe, Central limit theorems for urn models, Stochastic Process. Appl. 65 (1996), no. 1, 115-137.

[97] Richard P. Stanley, Enumerative combinatorics, vol. I, Wadsworth \& Brooks/Cole, 1986.

[98] Michael E. Taylor, Partial differential equations. I, Applied Mathematical Sciences, vol. 115, Springer-Verlag, New York, 1996.

[99] E. C. Titchmarsh, The theory of functions, second ed., Oxford University Press, 1939.

[100] Gérard Viennot, Une interpretation combinatoire des coefficients des développements en série entière des fonctions elliptiques de Jacobi, Journal of Combinatorial Theory, series A 29 (1980), 121-133.

[101] Jeffrey Scott Vitter and Philippe Flajolet, Analysis of algorithms and data structures, Handbook of Theoretical Computer Science (J. van Leeuwen, ed.), vol. A: Algorithms and Complexity, North Holland, 1990, pp. 431-524.

[102] J. Vuillemin, A unifying look at data structures, Communications of the ACM 23 (1980), no. 4, 229-239.

[103] E. T. Whittaker and G. N. Watson, A course of modern analysis, fourth ed., Cambridge University Press, 1927, Reprinted 1973.

[104] David Williams, Probability with martingales, Cambridge Mathematical Textbooks, Cambridge University Press, Cambridge, 1991.

[105] David Williams and Paul McIlroy, The OK Corral and the power of the law (a curious Poisson-kernel formula for a parabolic equation), The Bulletin of the London Mathematical Society 30 (1998), no. 2, 166-170.

[106] Andrew Chi Chih Yao, On random 2-3 trees, Acta Informatica 9 (1978), no. 2, 159-170.

[107] V. M. Zolotarev, One-dimensional stable distributions, American Mathematical Society, Providence, RI, 1986, Translated from the Russian by H. H. McFaden, Translation edited by Ben Silver. 